\title{
AN APERTURE-COUPLED STACKED MICROSTRIP ANTENNA FOR GPS FREQUENCY BANDS L1, L2, AND L5
}

BY

\section{EHSAN GHARIB DOUST}

\section{A THESIS SUBMITTED TO THE FACULTY OF GRADUATE STUDIES AND RESEARCH IN PARTIAL FULFILLMENT OF THE REQUIREMENTS FOR THE DEGREE OF MASTERS OF APPLIED SCIENCE}

OTTAWA-CARLETON INSTITUTE FOR ELECTRICAL AND COMPUTER ENGINEERING

\author{
DEPARTMENT OF ELECTRONICS \\ FACULTY OF ENGINEERING \\ CARLETON UNIVERSITY \\ OTTAWA, ONTARIO
}

(C) Ehsan Gharib Doust, 2006 


$\begin{array}{ll}\begin{array}{l}\text { Library and } \\ \text { Archives Canada }\end{array} & \begin{array}{l}\text { Bibliothèque et } \\ \text { Archives Canada }\end{array} \\ \begin{array}{l}\text { Published Heritage } \\ \text { Branch }\end{array} & \begin{array}{l}\text { Direction du } \\ \text { Patrimoine de l'édition }\end{array} \\ \begin{array}{l}\text { 395 Wellington Street } \\ \text { Ottawa ON K1A ON4 }\end{array} & \begin{array}{l}\text { 395, rue Wellington } \\ \text { Ottawa ON K1A ON4 } \\ \text { Canada }\end{array}\end{array}$

Your file Votre référence ISBN: 978-0-494-23337-5 Our file Notre référence ISBN: 978-0-494-23337-5

NOTICE:

The author has granted a nonexclusive license allowing Library and Archives Canada to reproduce, publish, archive, preserve, conserve, communicate to the public by telecommunication or on the Internet, loan, distribute and sell theses worldwide, for commercial or noncommercial purposes, in microform, paper, electronic and/or any other formats.

The author retains copyright ownership and moral rights in this thesis. Neither the thesis nor substantial extracts from it may be printed or otherwise reproduced without the author's permission.
AVIS:

L'auteur a accordé une licence non exclusive permettant à la Bibliothèque et Archives Canada de reproduire, publier, archiver, sauvegarder, conserver, transmettre au public par télécommunication ou par l'Internet, prêter, distribuer et vendre des thèses partout dans le monde, à des fins commerciales ou autres, sur support microforme, papier, électronique et/ou autres formats.

L'auteur conserve la propriété du droit d'auteur et des droits moraux qui protège cette thèse. $\mathrm{Ni}$ la thèse ni des extraits substantiels de celle-ci ne doivent être imprimés ou autrement reproduits sans son autorisation.
In compliance with the Canadian

Privacy Act some supporting forms may have been removed from this thesis.

While these forms may be included in the document page count, their removal does not represent any loss of content from the thesis.
Conformément à la loi canadienne sur la protection de la vie privée, quelques formulaires secondaires ont été enlevés de cette thèse.

Bien que ces formulaires aient inclus dans la pagination, il n'y aura aucun contenu manquant.

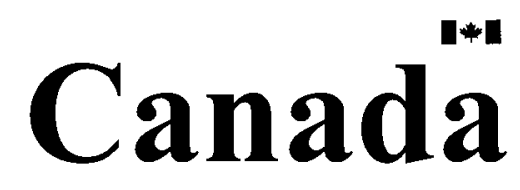




\section{To my parents,}

Who has been the source of life and love for me. 


\section{Abstract}

This thesis investigates a technique of stacking rectangular microstrip antenna elements to achieve dual frequency operation. In the particular configuration studied, two square patches are stacked on top of each other above a common ground plane. These elements are fed by the means of an aperture-coupled feed network. The aperture-coupled configuration has the advantage of isolating spurious feed radiation by the use of the common ground plane. The antenna was designed to operate at GPS frequencies L1, L2 and L5. Two similar antenna structures were designed using different substrate material. The reason for designing a second antenna was to reduce the dimensions of the first antenna for array configuration. 


\section{ACKNOWLEDMENTS}

First and foremost I would like to thank my thesis supervisor Prof. Jim Wight for giving me the opportunity to study under his direction for which I'm forever grateful; and for his support, encouragement and guidance during the course of this work and other aspects in life. I would also like to thank and my best friend and colleague, Varahram Hemmati, for his friendship and support during the past 5 years especially during the course of this work. I would also like to thanks Dr. Mike Vinnins and Dr. Michel Clenet of DRDC Ottawa, where this research was carried out, for their helpful support and encouragements. Last but not least I would like to thank all my family and friends, for their love and support throughout my life. 


\section{TABLE OF CONTENTS}

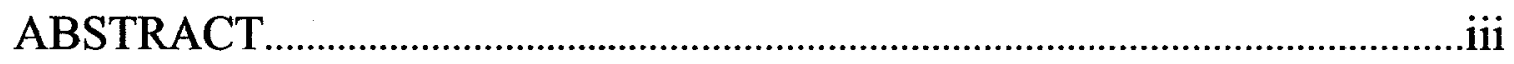

ACKNOWLEDGEMENTS..................................................................................... iv

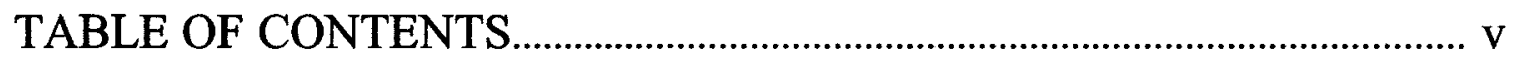

LIST OF FIGURES..........................................................................................viii

LIST OF TABLES .............................................................................................

LIST OF NOTATIONS AND ABBRIVIATIONS...............................................ii

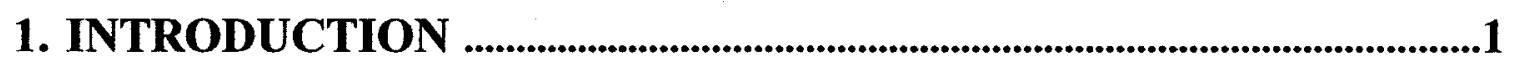

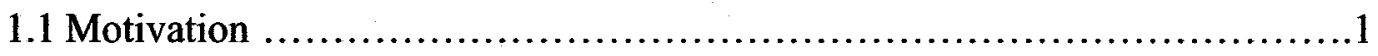

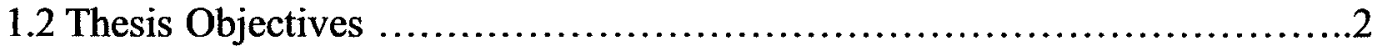

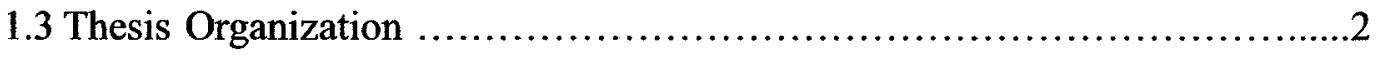

2. BACKGROUND INFORMATION.................................................................4

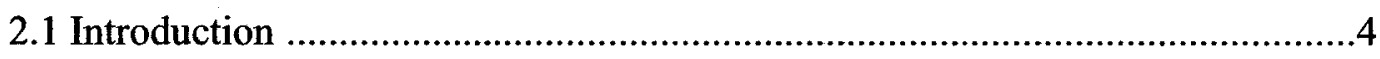

2.2 Global Positioning System Description and History ................................

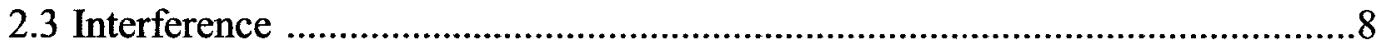

2.3.1 GPS Antenna Anti-Jamming Techniques ..................................10

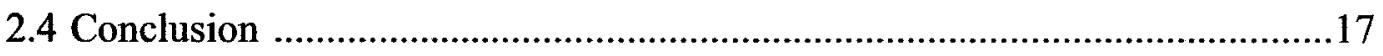




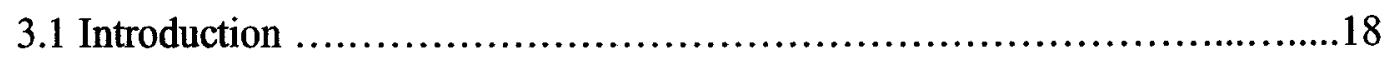

3.2 Rectangular Microstrip Antennas .........................................19

3.2.1 Radiation Mechanism .........................................19

3.3 Microstrip Antenna Analytical Models ..................................21

3.3.1 Transmission Line Model .........................................21

3.3.2 Cavity Model ....................................................................................25

3.4 Microstrip Antenna Design Considerations ......................................................29

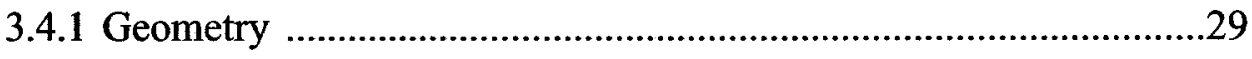

3.4.2 Directivity, Gain and Radiation Efficiency ......................................30

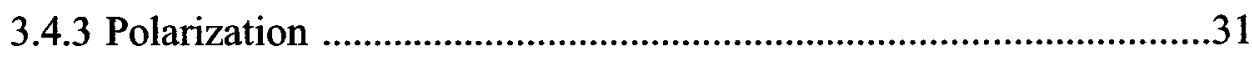

3.4.4 Radiation Pattern Characteristics .....................................................32

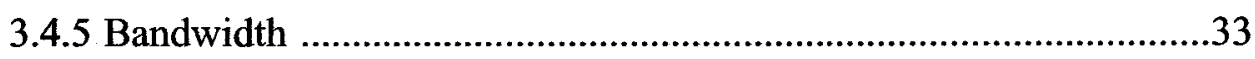

3.4.6 Effect of Finite Size Ground Plane ……………...............................35

3.5 Microstrip Antenna Feeding Techniques .......................................................36

3.5.1 Coaxial Probe Feeding .......................................................................

3.5.2 Coplanar Microstrip Line Feeding ….............................................38

3.5.3 Aperture Coupled Feeding ................................................................40

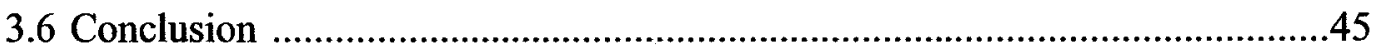

4. STACKED MICROSTRP ANTENNA DESIGN........................................47

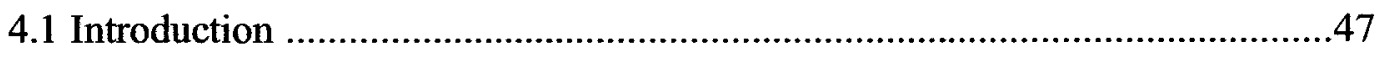

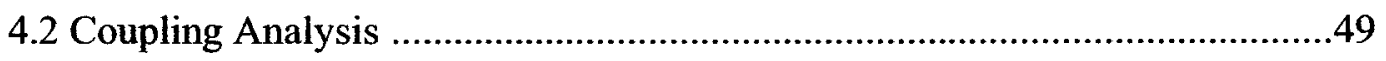

4.3 Studies on Aperture-Coupled Stacked Patch Antennas .................................50

vi 


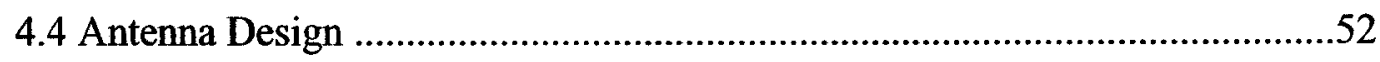

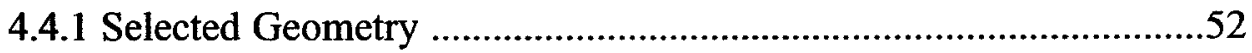

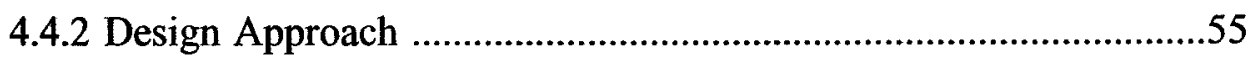

4.4.3 Feed Network Design .......................................................57

4.4.4 First Antenna Design and Simulation .....................................61

4.4.5 Second Antenna Design and Simulation ..................................76

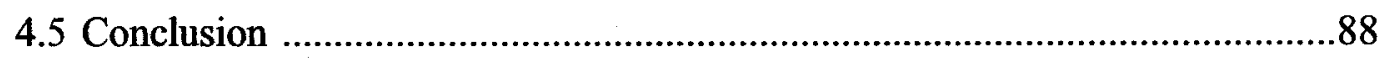

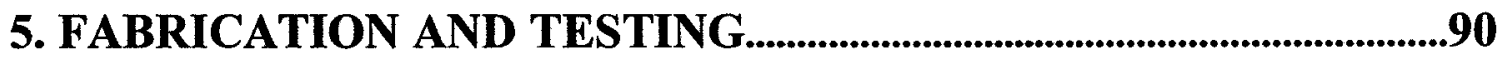

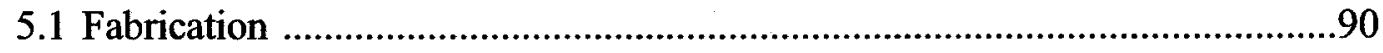

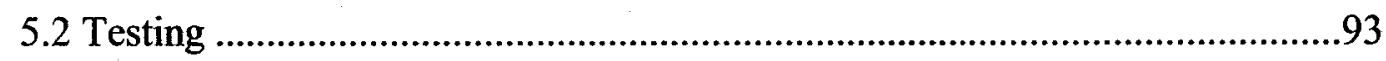

5.2.1 First Antenna Test Results ...................................................93

5.2.2 Second Antenna Test Results ..............................................101

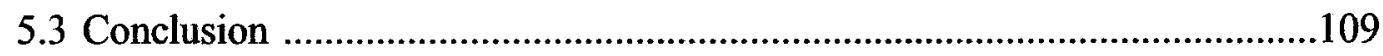

6. CONCLUSIONS AND FUTURE WORK.....................................110

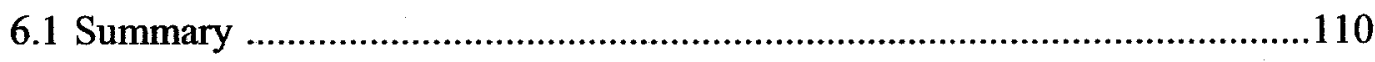

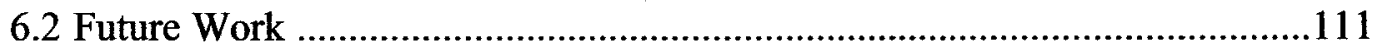

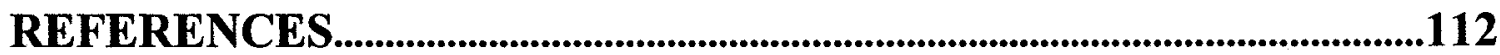

Appendix A: Measurement Setup ................................................................115

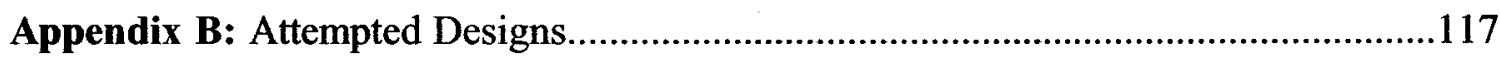

vii 


\section{LiST OF FiguRES}

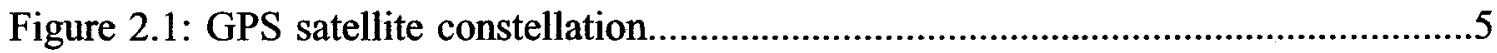

Figure 2.2: GPS signal Generation..................................................................

Figure 2.3: Antenna Anti-Jamming Structure.......................................................10

Figure 2.4: Desirable Properties of FRPAs........................................................11

Figure 2.5: Antenna electronic units for null-steering CRPAs..................................15

Figure 3.1: Rectangular Microstrip antenna configuration..........................................19

Figure 3.2: Microstrip antenna charge distribution and current density........................20

Figure 3.3: Fringing fields for the dominant mode in a rectangular microstrip patch......20

Figure 3.4: Equivalent admittance circuit model.....................................................22

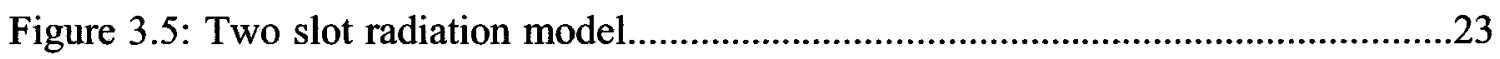

Figure 3.6: Electric field and magnetic current distributions for different modes.........26

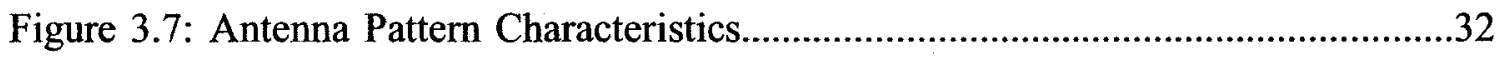

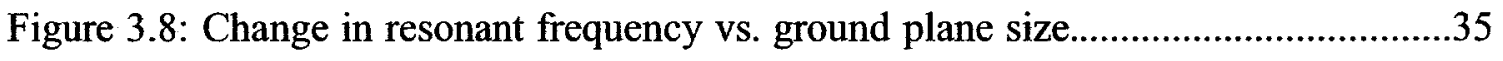

Figure 3.9: E-plane pattern for infinite vs. finite ground plane...................................36

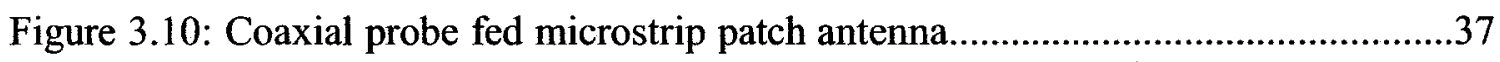

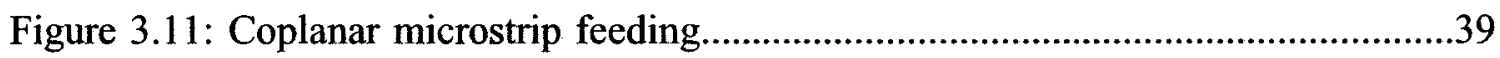

Figure 3.12: Aperture-coupled feeding technique.....................................................41

Figure 3.13: Simplified circuit of aperture-coupled patch..........................................42

Figure 3.14: Transmission line equivalent circuit of microstrip patch.........................43

Figure 4.1: Aperture-Coupled Stacked Microstrip Antenna Exploded Geometry...........48

viii 
Figure 4.2: Electric and magnetic field distribution for the even mode. .49

Figure 4.3: (a) Wide-centered feedline. (b) Dual-offset feedline.................................50

Figure 4.4: (a) Parallel feed configuration. (b)Series feed configuration.........................51

Figure 4.5: Selected geometry of the aperture-coupled stacked GPS antenna................54

Figure 4.6: Typical impedance locus of an aperture-coupled stacked patch antenna.......55

Figure 4.7: Microstrip feeding for RHCP stacked-patch with cross aperture.................57

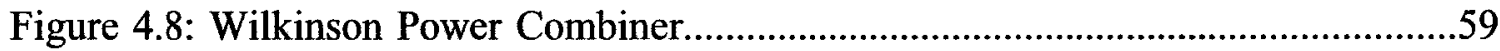

Figure 4.9: Axial Ratio vs. Power percentage at an input of a Wilkinson.......................59

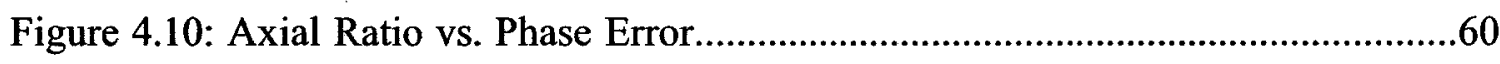

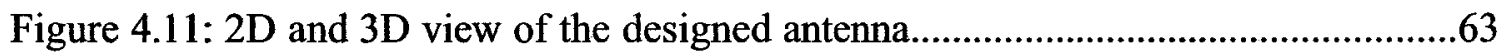

Figure 4.12: Simulated return loss of the first antenna measured at the input port.........64

Figure 4.13: Simulated impedance locus of the first antenna...................................65

Figure 4.14: Simulated gain of the first antenna measured at the input port.................66

Figure 4.15: Simulated axial ratio vs. frequency of the first design at broadside..........67

Figure 4.16: Simulated axial ratio vs. theta in $\phi=0$ plane. (a) $\mathrm{L} 1$, (b) $\mathrm{L} 2$, (c) L5........69

Figure 4.17: Simulated radiation pattern for L1; (a) $\phi=0$, (b) $\phi=90 \ldots \ldots \ldots \ldots \ldots \ldots \ldots . . . .71$

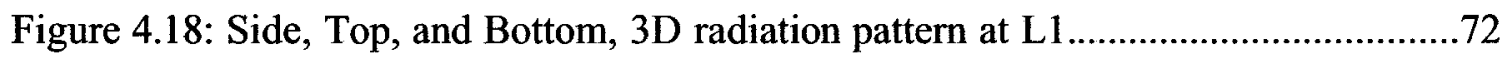

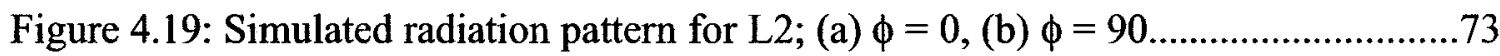

Figure 4.20: Simulated radiation pattern for L5; (a) $\phi=0$, (b) $\phi=90 \ldots \ldots \ldots \ldots \ldots \ldots \ldots . . . . .74$

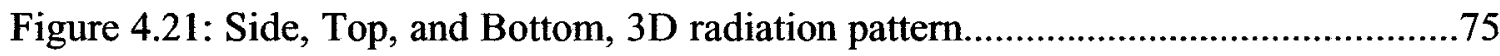

Figure 4.22: Simulated Return Loss of the second antenna measured at the input..........78

Figure 4.23: Simulated impedance locus of the second antenna................................79

Figure 4.24: Simulated gain of the second antenna measured at the input port..............80 
Figure 4.25: Simulated axial ratio vs. frequency of the second design at broadside. .81

Figure 4.26: Simulated axial ratio vs. theta in $\phi=0$ plane; (a) $\mathrm{L} 1$, (b) $\mathrm{L} 2$, (c) L5..........83

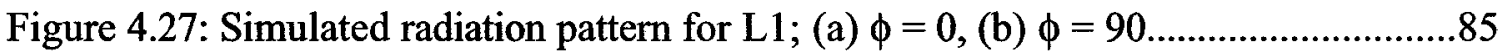

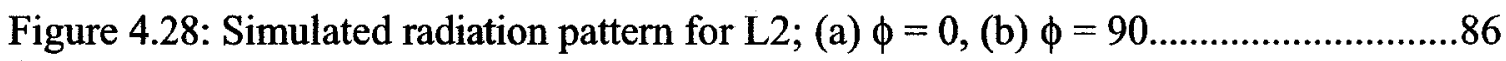

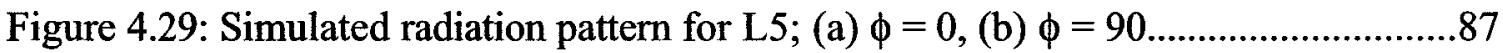

Figure 5.1: Fabricated aperture-coupled stacked patch antenna (Antenna 1)................91

Figure 5.2: Fabricated aperture-coupled stacked patch antenna (Antenna 2)..................92

Figure 5.3: Tested return loss of the first antenna................................................93

Figure 5.4: First Antenna radiation pattern for $L 1$; (a) $\phi=0$, (b) $\phi=90 \ldots \ldots \ldots \ldots \ldots \ldots \ldots . . . .95$

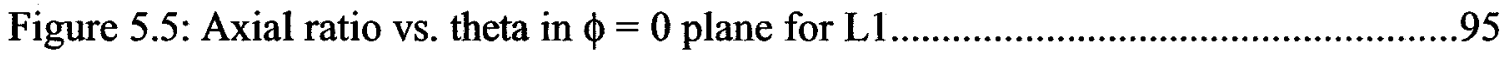

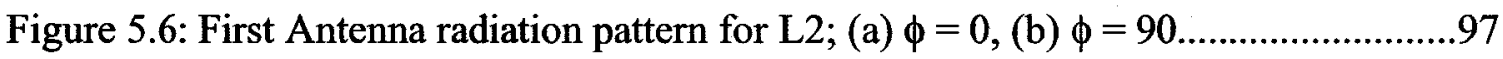

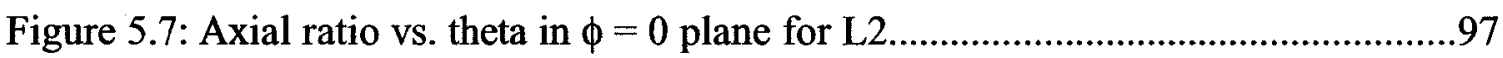

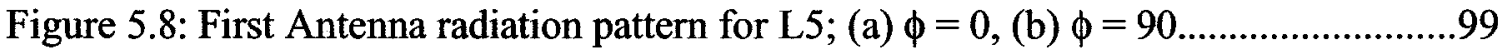

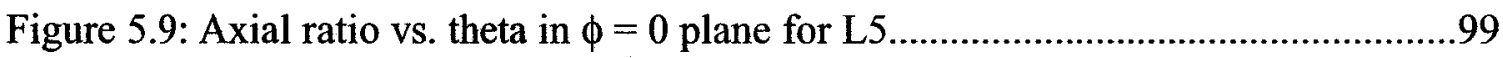

Figure 5.10: Tested return loss of the second antenna........................................101

Figure 5.11: Second Antenna radiation pattern for $\mathrm{L1}$; (a) $\phi=0$, (b) $\phi=90 \ldots \ldots \ldots \ldots \ldots 103$

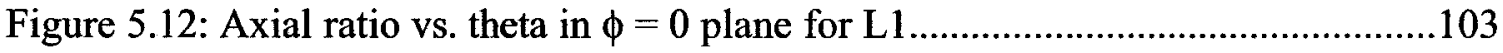

Figure 5.13: Second Antenna radiation pattern for $L 2 ;$ (a) $\phi=0$, (b) $\phi=90 \ldots \ldots \ldots \ldots \ldots 105$

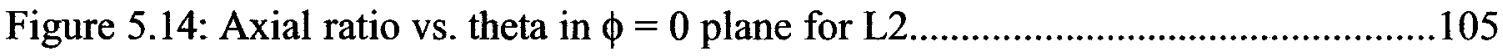

Figure 5.15: Second Antenna radiation pattern for $\mathrm{L} 5 ;(\mathrm{a}) \phi=0$, (b) $\phi=90 \ldots \ldots \ldots \ldots \ldots . . . .107$

Figure 5.16: Axial ratio vs. theta in $\phi=0$ plane for L5 ....................................107 


\section{LIST OF TABLES}

Table 2.1: Antenna anti-jamming techniques summary.......................................16

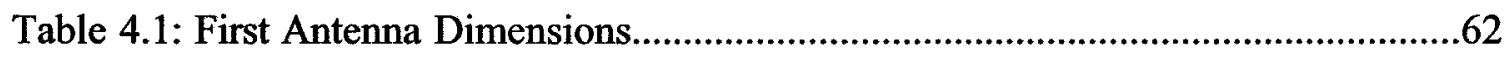

Table 4.2: Second Antenna Dimensions............................................................... 77

Table 6.1: Comparison of simulation and test results of first antenna..........................100

Table 6.2: Comparison of simulation and test results of second antenna.....................108 


\section{LiST OF NOTATIONS AND}

\section{Abbriviations}

\begin{tabular}{|c|c|}
\hline$\varepsilon_{\mathrm{r}}$ & Relative Dielectric Constant \\
\hline$\varepsilon_{\text {eff }}$ & Effective Dielectric Constant \\
\hline h & Dielectric Substrate Thickness \\
\hline $\mathrm{k}$ & Wave Number \\
\hline$\lambda_{0}$ & Free Space Wavelength \\
\hline$\lambda_{\mathrm{g}}$ & Guided Wavelength \\
\hline $\mathbf{L}$ & Length of Microstrip Antenna \\
\hline $\mathbf{L}_{\mathbf{s}}$ & Slot Length \\
\hline $\mathrm{L}_{\text {stub }}$ & Stub Length \\
\hline$Q_{r}$ & Radiation Quality Factor \\
\hline $\tan \delta$ & Dielectric Loss Tangent \\
\hline $\mathrm{W}_{\mathrm{s}}$ & Slot Width \\
\hline W & Width of Microstrip Antenna \\
\hline GPS & Global Positioning System \\
\hline SPS & Standard Positioning System \\
\hline PPS & Precision Positioning System \\
\hline DGPS & Differential Global Positioning System \\
\hline C/A Code & Course Acquisition Code \\
\hline
\end{tabular}

xii 


$\begin{array}{ll}\text { P Code } & \text { Precise Code } \\ \text { CDMA } & \text { Code Division Multiple Access } \\ \text { NAVSTAR } & \text { Navigation Signal Timing and Ranging } \\ \text { ISU } & \text { Interference Suppression Unit } \\ \text { FRPA } & \text { Fixed Radiation Pattern Antenna } \\ \text { BW } & \text { Band Width } \\ \text { AR } & \text { Axial Ratio } \\ \text { RHCP } & \text { Right Hand Circularly Polarized } \\ \text { LHCP } & \text { Left Hand Circularly Polarized } \\ \text { DOA } & \text { Direction of Arrival } \\ \text { CRPA } & \text { Controlled Radiation Pattern Antenna } \\ \text { FPBW } & \text { Half Power Beam Width } \\ \text { FNBW } & \text { First Null Beam Width } \\ \text { FBR } & \text { Front to Back Ratio } \\ \text { CPBW } & \text { Circular Polarized Beam Width } \\ \text { VSWR } & \text { Voltage Standing Wav Ratio } \\ \text { FHesistant Type 4 }\end{array}$

xiii 


\section{Chapter 1}

\section{INTRODUCTION}

\subsection{Motivation}

The applications of microstrip antennas have grown rapidly in the past decades. Microstrip antennas have numerous advantages, they are light in weight, they can be designed to operate over a large range of frequencies, they can easily be combined to form linear or planar arrays, and they can generate linear, dual, and circular polarizations. These antennas are inexpensive to fabricate using printed circuit board etching, which makes them very useful for integrated active antennas. These antennas have successfully been engaged in applications such as cellular and satellite communications, radars, and GPS receivers. However, the narrow bandwidth (1-5\%) of microstrip antennas restricts their application to single, narrowband frequencies [1].

This thesis describes work directed toward the design of an aperture-coupled stacked microstrip antenna for GPS frequency bands L1, L2, and L5. This configuration overcomes the limitations associated with microstrip antennas without increasing the area occupied by the element. 


\subsection{Thesis Objectives}

Stacked microstrip patch configuration can be used to achieve dual or multiple frequency operation. Also, it has been shown that stacking microstrip patches result in an increased impedance bandwidth over a single layer radiator. This thesis addresses the design procedure of a dual-band aperture-coupled stacked patch antenna in GPS frequencies $\mathrm{L} 1$ and $\mathrm{L} 2 / \mathrm{L} 5$.

This antenna was designed so that the first band located at $\mathrm{Ll}=1575.42 \mathrm{MHz}$ has an impedance bandwidth of $20 \mathrm{MHz}$ to $50 \mathrm{MHz}(2-4 \%)$; and the second band located at $\mathrm{L} 2$ $=1227.60 \mathrm{MHz}$ and $\mathrm{L} 5=1176.45 \mathrm{MHz}$ is wide enough to cover both frequencies and has an impedance bandwidth of $70 \mathrm{MHz}$ to $150 \mathrm{MHz}(6-12.5 \%)$. It is also desirable to achieve a high rejection level at other frequency bands. These antennas are also right hand circularly polarized $(\mathrm{RHCP})$, so the axial ratio bandwidth $(\mathrm{AR}<3 \mathrm{~dB})$ should be enough to cover all desired frequency bands. Two similar antenna structures were designed using different substrate material. The first antenna was designed so that the fabrication and material cost is minimal. The reason for designing a second antenna was to reduce the dimensions of the first antenna at the cost of more expensive material with higher permittivity.

\subsection{Thesis Organization}

This thesis is divided into 6 chapters and is organized as follows:

Chapter 1 contains the thesis introduction

Chapter 2 provides a basic description of the Global Positioning System (GPS), 
concept of interference, jamming, anti-jamming, and the main methods of antenna anti-jamming.

Chapter 3 includes an overview of microstrip antennas radiation mechanism, modeling, design, and feeding techniques.

Chapter 4 describes design procedure of a dual-band aperture-coupled stacked patch antenna in GPS frequencies L1 and L2/L5. Simulation results are also included in this chapter.

Chapter 5 presents the fabrication and testing of the two antennas. Comparison to the simulation results is also included in this chapter.

Chapter 6 provides a summary of the work presented and possible opportunity for future work. 


\section{Chapter 2}

\section{BACKGROUND INFORMATION}

\subsection{Introduction}

The first part of this chapter will provide a basic description of the Global Positioning System, its operation and history. The second part investigates the concept of interference, jamming, anti-jamming, and the main methods of antenna anti-jamming. The antenna anti-jamming methods are described in more detail because of its relevance to the designed antennas.

\subsection{Global Positioning System Description and History}

The Global Positioning System (GPS) is a satellite-based navigation system formed by a constellation of 29 well-spaced satellites (as of August 2006) in six intermediate circular orbits. For the global coverage 24 satellites are enough (four on each orbital plane) and the rest are spare in case of any failure. The orbits are aligned so that at least four satellites are in the line of sight from anywhere on earth. These satellites orbit the earth twice a day at the altitude of 26,560 kilometers [4]. 
The GPS is owned and operated by the U.S. Department of Defense (DOD) and was officially named NAVSTAR GPS (Navigation Signal Timing and Ranging Global Positioning System) and is available for general use around the world. Depending on the type of receiver being used, the location accuracy is anywhere from 1 to 100 meters. Prior to the development of the GPS system, the first satellite system was called Transit and was operational in 1964. Due to lack of a timing device, it took a receiver 15 minutes to calculate its position. GPS is a great improvement over the Transit system. The original use of GPS was as a military positioning, navigation, and weapons aiming system to replace not only Transit, but other navigation systems as well. The first GPS satellite was launched in 1978 and the first products for civilian consumers appeared in the mid 1980's. In 1984 a portion of GPS capabilities was made available to the civil community. The system is still being improved and new, better satellites are still being launched to replace older ones.

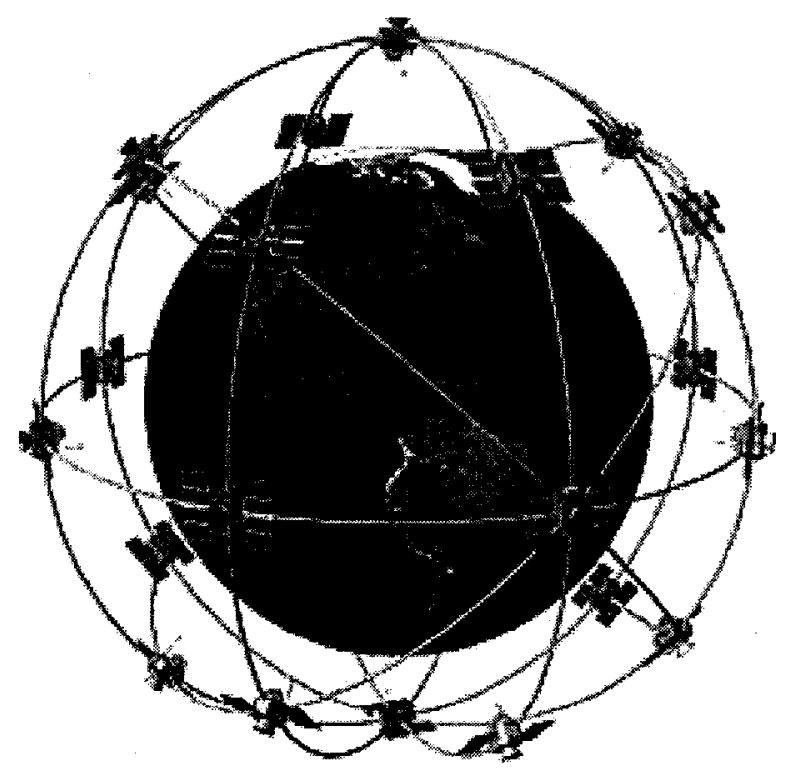

Figure 2.1: GPS satellite constellation 
Each satellite is equipped with an atomic clock and continually broadcasts its changing position and time. The satellites broadcast two forms of accurate clock information, the Coarse Acquisition (C/A) code, which is used for Standard Positioning Service (SPS), and the Precise (P) code, which is used for Precision Positioning Service (PPS). The C/A-code is usually used for most civilian navigation purposes and consists of a 1,023 bit long pseudo-random code broadcast at $1.023 \mathrm{MHz}$ with $2 \mathrm{MHz}$ bandwidth, repeating every millisecond. The $\mathrm{C} / \mathrm{A}$-code is different for each satellite, which allows them to be identified. The P-code is similar but broadcasts at $10.23 \mathrm{MHz}$ with $20 \mathrm{MHz}$ bandwidth, and repeats once a week. In normal operation mode the P-code is first encrypted into the $\mathrm{Y}$-code $(\mathrm{P}(\mathrm{Y}))$, which can only be decrypted with a valid decryption key. P-code is also unique for each satellite [4].

In addition to $\mathrm{C} / \mathrm{A}$ and $\mathrm{P}(\mathrm{Y})$ codes, the signal is also modulated with a $50 \mathrm{bit} / \mathrm{sec}$ navigation message. This Navigation Message contains coarse time information with second precision along with status information about the satellites (almanac). It also includes orbital information that allows the receiver to calculate the position of the satellite at any point in time (ephemeris). GPS uses direct sequence spread spectrum Code Division Multiple Access (CDMA) since all satellites transmit at the same carrier frequencies but with different spreading codes. All three signals, Navigation Message, $\mathrm{C} / \mathrm{A}$ and $\mathrm{P}(\mathrm{Y})$ codes, are mixed together and sent on the primary carrier frequency, $\mathrm{L}$, at 1575.42 MHz. The C/A-code is modulated on a carrier that is $90^{\circ}$ out of phase from the $\mathrm{P}$-code carrier. The $\mathrm{P}(\mathrm{Y})$ signal is also sent alone on the carrier frequency, $\mathrm{L} 2$, at 1227.60 $\mathrm{MHz}$ [10]. The GPS signal generation flowchart is shown in Figure 2.2. 


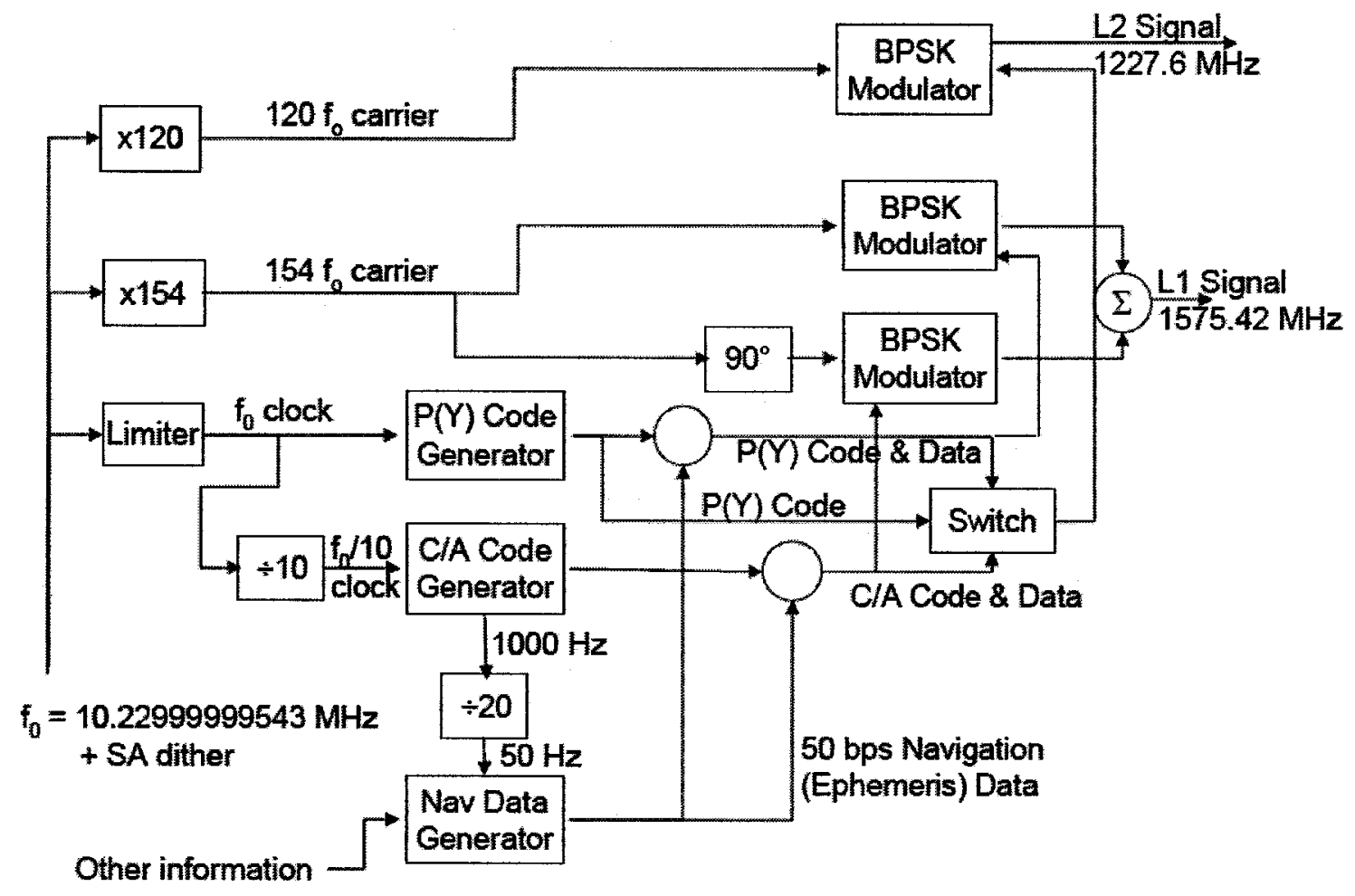

Figure 2.2: GPS signal Generation [10].

GPS carrier frequency, L5, at $1176.45 \mathrm{MHz}$ is being proposed for the use as a civilian Safety of Life (SOL) signal. This frequency is in an internationally protected range for aeronautical navigation, with little or no interference under all circumstances. This service is expected to be operational by 2008 . Also a new civilian GPS signal called L2C was introduced in late 2005 , offering enhanced accuracy and reliability.

There are some factors, which degrade GPS accuracy [4]. GPS signals must travel through the ionosphere, and depending on the local electron density and thickness of ionosphere the speed of wave modulation is reduced. This effect known as Ionospheric delay causes interruption in signal arrival and produces an error of about $10 \mathrm{~m}$. Also due to the fact that the refractive index of the lower atmosphere is not exactly $1(\sim 1.0003)$, a 
delay of about 0.5 meter is introduced, known as Tropospheric delay. Selected Availability (SA) is another source of error, which is introduced intentionally into publicly available navigation signals. This error makes it difficult to use GPS for guiding long range missiles to precise targets. This error consists of clock offsets, which can produce error up to 100 meters. Even without SA the satellite positions are in error by 4 meters, which is known as Ephemeris error. GPS signals can also be affected by multipath issues, where the radio signals reflect off surrounding terrain, buildings, etc.

There are several methods to enhance the GPS accuracy. The most common and popular method is called Differential GPS (DGPS), which can improve the normal GPS accuracy of 4-20 meters to 1-3 meters [4]. DGPS uses a series of stationary GPS receivers with fixed, known position. These receivers calculate the difference between their actual position and the position calculated by the received GPS signal. The difference is transmitted as a local FM signal, allowing many civilian GPS receivers to fix the signal for improved accuracy.

\subsection{Interference}

Interfering with any radio navigation system, including satellite based navigation, is possible. This interference may be intentional and should be anticipated for military receivers or unintentional. There has also been at least one documented case of unintentional jamming, tracing back to a malfunctioning TV antenna preamplifier [8]. If stronger signals were generated intentionally, they could potentially interfere with aviation GPS receivers within line of sight. Low power jammers would have limited 
military usefulness and high power jammers would be easy to locate and destroy. The typical types of radio frequency interference along with their sources are listed below [4]:

1. Broad-band Gaussian Jammers: The typical source for this type of interference is intentional noise jammers.

2. Wide-band or narrow-band phase/frequency modulation: TV transmitter's harmonics or near-band microwave link transmitters could be sources of wide-band phase/frequency modulated interference. AM transmiter's harmonics or CB (Civilian Band) transmitter's harmonics could cause phase/frequency modulated interference.

3. Wide-band spread Spectrum: The typical source for this type of interference is intentional spread spectrum jammers or near-field of pseudolites (pseudo-satellite).

4. Wide-band Pulse Jammers: Radar transmitters are typical source of this kind of interference.

5. Narrow-band swept continuous wave $(\mathrm{CW})$ : FM stations transmitter's harmonics could cause this type of interference.

6. Narrow-band $C W$ : Near-band unmodulated transmitter's carriers are typical source of this type of interference.

Anti-jam enhancements can be applied either at the antenna, between the antenna and the GPS receiver, or within the actual GPS receiver itself. Here we study the antenna level anti-jamming techniques. 


\subsubsection{GPS Antenna Anti-Jamming Techniques}

This section gives an overview of the most common antenna technologies for interference rejection in GPS. To further protect the GPS receiver from intentional jammers, additional anti-jamming enhancements can be applied at the antenna level. A basic antenna anti-jamming structure is shown below:

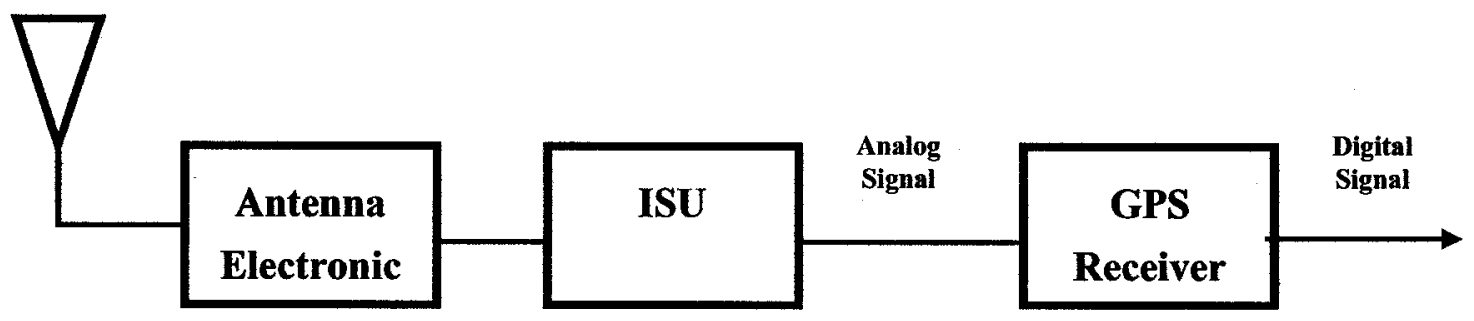

Figure 2.3: Antenna Anti-Jamming Structure

In this figure Antenna Electronics (AE) refers to dedicated electronic units that are required by a particular antenna enhancement. These units usually have several analog inputs and a single analog output. The Interference Suppression Units (ISU) is an optional electronics unit that can be cascaded with current GPS receivers. These units are mainly applicable to Fixed Radiation Pattern Antennas (FRPA) and can be used in most current GPS installations at relatively low cost. These units have a single analog input and output and further increase the anti-jam against narrow band jammers.

A single element or a multiple element antenna can be used for the purpose of anti-jamming. The single element antenna itself could be either an FRPA or an adaptive dual polarization antenna. FRPAs are single element GPS antennas currently used with 
most GPS receivers. These antennas connect directly to the GPS receiver, without the need for an antenna electronics unit. FRPAs can be effective against all low elevation jammers by designing them such that they have a null near the horizon. The depth of null that can be achieved limits the amount of anti-jamming. Deeper nulls also reduce satellite coverage due to the limitations on the steepness of the sides of the null. A low elevation null is also effective against ground reflected multi-path signals. FRPAs can be cascaded with a number of ISUs to obtain extra anti-jamming against narrow-band, pulsed, and out of band jammers. These ISUs include PIN diodes, band-pass filters, analog adaptive filters and digital signal processing (DSP) techniques. The desirable radiation pattern of an FRPA is shown in Figure 2.4, followed by its description [8]:

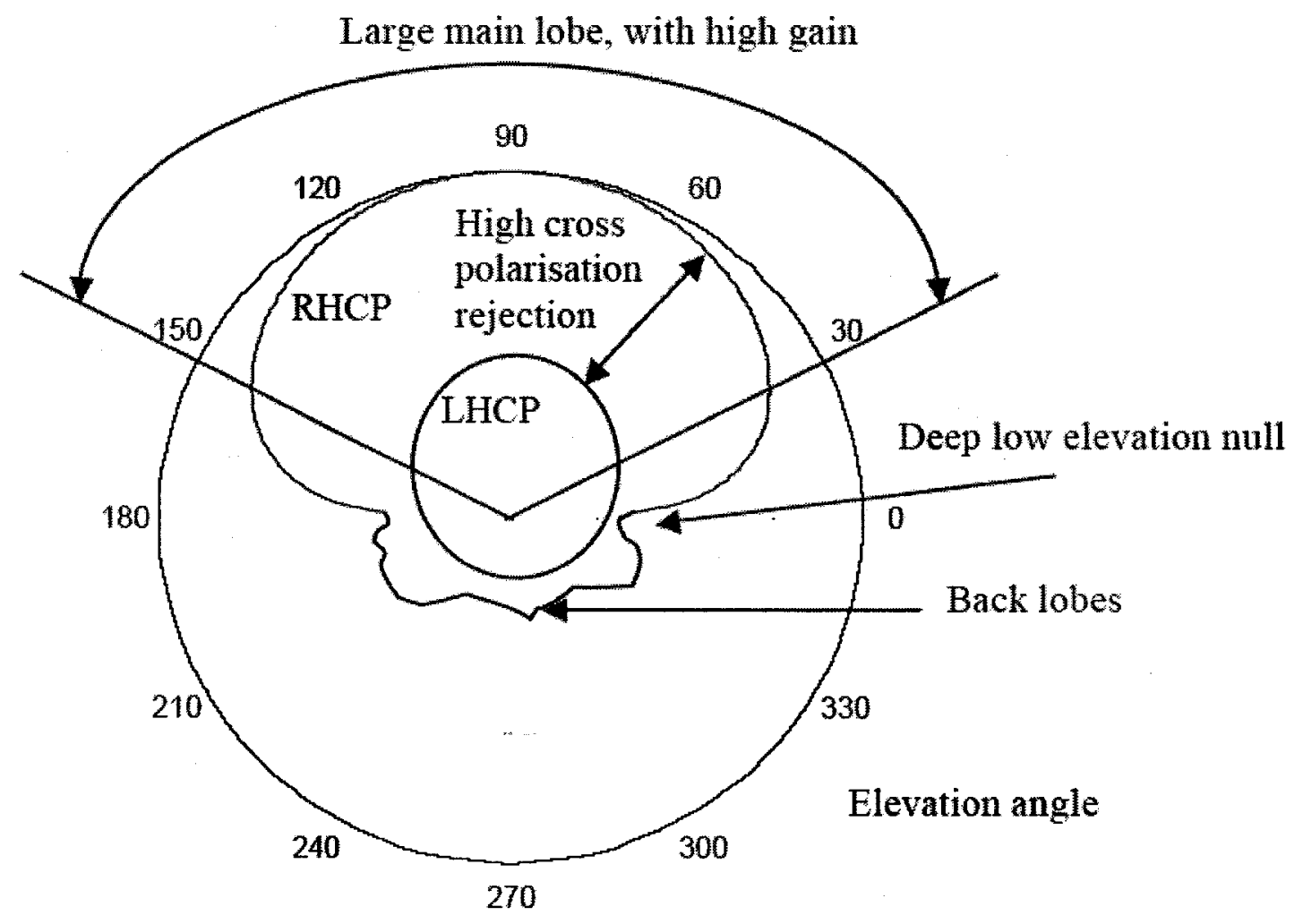

Figure 2.4: Desirable Properties of FRPAs [8] 
1. Broad main lobe over the upper hemisphere: To ensures high satellite availability.

2. High gain for RHCP signals from upper hemisphere: The Signal to Noise Ratio SNR at the output of the antenna is directly proportional to the gain of the antenna in the direction of the desired signal.

3. A deep null in the radiation pattern near the horizon: This is useful for attenuating low elevation jammers and multi-path signals.

4. High cross polarization rejection: To attenuate left-hand circularly polarized (LHCP) signals. Multi-path signals are most likely to be LHCP since the polarization of signals changes from RHCP to LHCP after reflection.

5. Low back lobes: To provide extra protection against jammers or GPS multi-path signals on an airborne platform.

The two main conflicting criteria that need to be considered for GPS anti-jam applications are the depth of the low elevation null and the width of the main lobe. To optimize this trade off, the radiation pattern of the FRPA needs to have a sharp roll-off at low elevation angles. Microstrip patch antennas have a very similar radiation pattern, which makes them applicable for FRPAs, and will be discussed in the next chapters. Experimental results show that the roll off rates of up to $1 \mathrm{~dB} /$ degree can be achieved near the horizon [8].

Adaptive polarization antennas use a single antenna that picks up each polarization independently. The two polarizations are then adaptively combined to cancel out the jamming signal. This technique uses a small antenna element, the same size as a 
standard FRPA, together with an antenna electronics unit. This technique takes advantage of the polarization diversity between the GPS signal (RHCP) and the jamming signal, and is effective against all jammer waveforms and Directions of Arrivals (DOAs). Adaptive polarization technique is mainly applicable to low elevation anti-jamming since it is difficult to maintain RHCP for low elevation jammers (terrain effects, as well as body masking tend to destroy the RHCP structure of a signal). Also this technique is insensitive to the azimuth angle of the jammer and can reject multiple jammers. Single antenna techniques are very attractive for GPS anti-jamming applications, due to their small size, weight, simplicity, and cost. They are mainly applicable against low elevation jammers and are not as versatile as multi-element techniques.

Anti-jamming using multiple antennas is more costly and physically larger but can provide more anti-jamming capabilities than the single element antennas. Generally speaking this technique can be split into two main categories: Techniques using multiple FRPAs and adaptive antenna arrays, or Controlled Radiation Pattern Antennas (CRPAs) [9]. Multiple FRPA techniques either select between different FRPAs to optimize the antenna radiation pattern (switched antenna), or combine the signal from two different FRPAs to cancel the jammer (Multi-element cancellers). In the switched antenna technique several FRPAs are installed on a platform, and each FRPA is oriented in a different direction and covers a different portion of the sky. The signals from several FRPAs can be switched in or out to control antenna coverage. This technique may be expensive and there is limited flexibility in how the overall radiation pattern can be customized. 
Multi-element cancellers use a primary FRPA directed at the GPS satellites, and a secondary FRPA directed towards the jammer. The primary FRPA picks up the combination of GPS and jamming signal, while the secondary antenna picks up mainly the jamming signal. The two signals are then combined with the correct amplitude and phase, such that the jamming signal is cancelled. In this technique, distortion or cancellation of the desired GPS signal may happen where the secondary antenna picks up the GPS signals. This technique is mainly applicable for a single, low elevation jammer in airborne applications with the primary antenna located on top, and the secondary antenna on the bottom of the aircraft.

Controlled Radiation Pattern Antennas (CRPAs) continuously adjust the radiation pattern of an antenna array. CRPAs can steer nulls towards the jammer or combine beam and null steering to steer nulls towards the jammer and the main beam towards the satellites. Null-steering CRPAs use an array of patch antennas, connected to a digital or analog beamformer. Spatial nulls can be steered in any direction by adjusting the amplitude and phase of the beamformer coefficients. These nulls are effective against all kinds of jamming signals and polarizations. Null-steering arrays attempt to maintain a uniform beam pattern at all other angles. In an antenna array N-1 nulls can be steered towards jammers, where $\mathrm{N}$ is the number of elements [9]. The main limitation of null steering CRPAs is the possible loss of satellite signals that fall within the spatial nulls. This loss factor increases with the number of nulls being steered. Another potential problem is the inability of these techniques to preserve the phase of the GPS signal. This is important for carrier-phase differential applications. Also, null-steering CRPAs require 
a large antenna array and an additional electronics beamformer, both of which are expensive. However, due their ability to cancel multiple broad-band noise jammers from almost all DOAs, null steering CRPAs are generally considered to be the most effective antenna anti-jamming technology. Further improvements can be made to the null-steering CRPA by also steering the main beams towards the desired GPS satellites. A combined beam and null steering CRPA overcomes some of the limitations of null-steering CRPAs discussed above. These techniques use the same antenna array, but the antenna electronics unit is considerably more complicated, and contains several beamformers each dedicated to a different GPS satellite. Beam/Null-steering is achieved by adjusting the beamformer weights such the desired signal is in phase on each channel, the main beam is towards the satellites, and nulls are towards the jammer [9]. Using this approach, the jammer DOA does not affect the design. The following figure shows the antenna electronic units for null-steering CRPAs [8]:

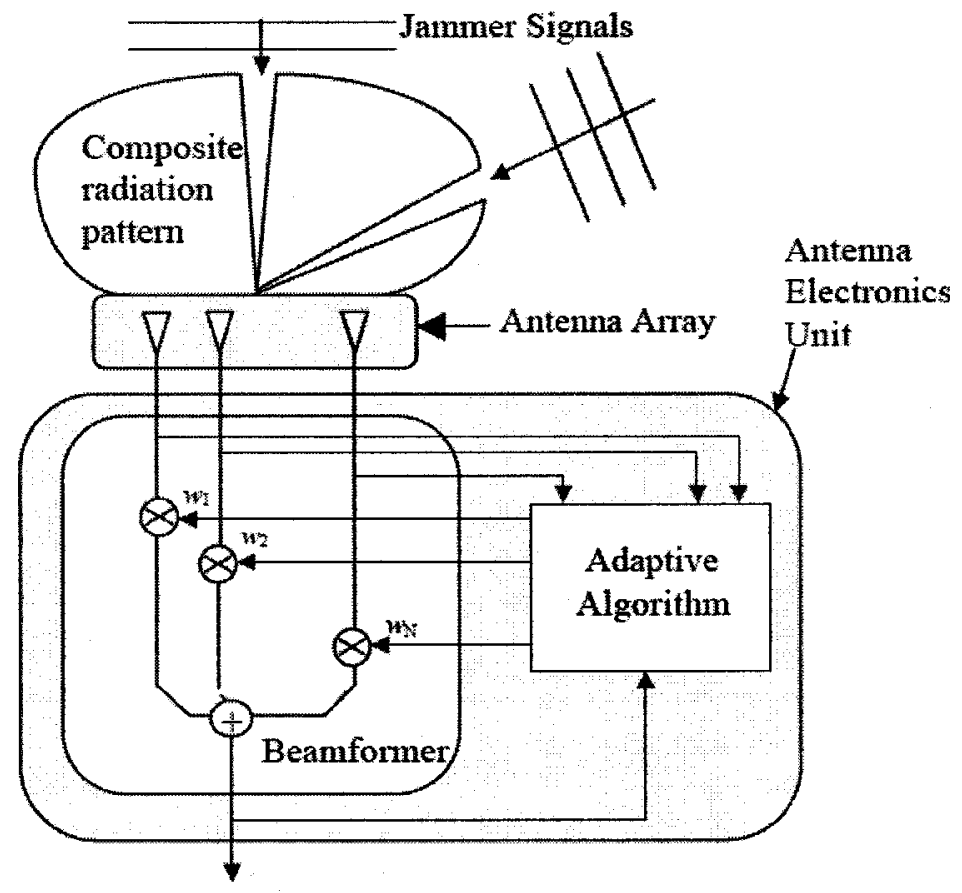

Figure 2.5: Antenna electronic units for null-steering CRPAs [8] 
Properties of antenna anti-jamming techniques are summarized in the following table [8]:

\begin{tabular}{|c|c|c|c|c|}
\hline \multicolumn{2}{|c|}{ Technique } & \multirow{2}{*}{\begin{tabular}{|l|} 
Implementation \\
Connects directly to \\
receiver. No antenna \\
electronic unit required \\
and can be optionally \\
cascaded with a number \\
of ISUs.
\end{tabular}} & \multirow{2}{*}{$\begin{array}{l}\text { Performance } \\
\text { Provides additional anti-jam against } \\
\text { any number of low elevation } \\
\text { jamming signals. Sensitive to } \\
\text { platform altitude. }\end{array}$} & \multirow{2}{*}{\begin{tabular}{|l|} 
Cost/Size \\
Low/Small
\end{tabular}} \\
\hline $\begin{array}{l}\text { Single } \\
\text { Antenna }\end{array}$ & FRPA & & & \\
\hline & $\begin{array}{l}\text { Adaptive } \\
\text { Dual } \\
\text { Polarization }\end{array}$ & $\begin{array}{l}\text { Requires an antenna } \\
\text { electronics unit Suitable } \\
\text { for hand-held receivers. }\end{array}$ & $\begin{array}{l}\text { Provides additional anti-jam against } \\
\text { multiple RHCP low elevation } \\
\text { jammers, and any additional } \\
\text { jammers that are not RHCP. } \\
\text { Sensitive to platform altitude }\end{array}$ & \\
\hline \multirow[t]{4}{*}{$\begin{array}{l}\text { Multiple } \\
\text { Antenna }\end{array}$} & $\begin{array}{l}\text { Switched } \\
\text { FRPA }\end{array}$ & $\begin{array}{l}\text { Several antennas } \\
\text { installed on a platform. } \\
\text { Requires an antenna } \\
\text { electronics unit to } \\
\text { switch between } \\
\text { antennas }\end{array}$ & $\begin{array}{l}\text { Provides additional anti-jam against } \\
\text { one or several jammers. } \\
\text { Performance depends on jammer } \\
\text { DOA and the relative orientation } \\
\text { and radiation pattern of each FRPA. } \\
\text { Has limited flexibility in adjusting } \\
\text { the overall radiation pattern, } \\
\text { compared with CRPAs. }\end{array}$ & Med/Large \\
\hline & $\begin{array}{l}\text { Multi- } \\
\text { Element } \\
\text { Canceller }\end{array}$ & $\begin{array}{l}\text { Requires two antenna } \\
\text { elements, one need to } \\
\text { be oriented towards the } \\
\text { satellites and the other } \\
\text { towards the jamming } \\
\text { signal. Thus, the } \\
\text { approximate jammer } \\
\text { DOA needs to be } \\
\text { known. In order to } \\
\text { combine signals an } \\
\text { antenna electronic unit } \\
\text { is required. }\end{array}$ & $\begin{array}{l}\text { Mainly applicable to airborne } \\
\text { platforms, where it provides } \\
\text { additional anti-jam against low } \\
\text { elevation jammers and multi-path. } \\
\text { Not effective if the GPS signal and } \\
\text { jammer are close together in angle. } \\
\text { This technique is sensitive to the } \\
\text { attitude of the platform. Mainly } \\
\text { applicable to a single jammer, but in } \\
\text { some cases it can reject multiple } \\
\text { jammers near the horizon }\end{array}$ & \multirow[t]{3}{*}{ High/Large } \\
\hline & $\begin{array}{l}\text { Null- } \\
\text { Steering } \\
\text { CPRA }\end{array}$ & $\begin{array}{l}\text { Requires a relatively } \\
\text { large antenna array and } \\
\text { antenna electronics unit. } \\
\text { Spatial nulls can be } \\
\text { steered in any direction } \\
\text { by adjusting the } \\
\text { amplitude and phase of } \\
\text { the beamformer } \\
\text { coefficients }\end{array}$ & $\begin{array}{l}\text { Provides significant anti-jam } \\
\text { margins against multiple (N-1) } \\
\text { broad-band jammers from any } \\
\text { direction by steering nulls in angle. } \\
\text { Nulls also reduce satellite visibility. }\end{array}$ & \\
\hline & $\begin{array}{l}\text { Beam/Null- } \\
\text { steering } \\
\text { CPRA }\end{array}$ & $\begin{array}{l}\text { Requires a relatively } \\
\text { large antenna array and } \\
\text { antenna electronics unit. } \\
\text { Beam/Null-steering is } \\
\text { achieved by adjusting } \\
\text { the beamformer weights } \\
\text { such main beam is } \\
\text { towards the satellites, } \\
\text { and nulls are towards } \\
\text { the jammer. }\end{array}$ & $\begin{array}{l}\text { Provides significant anti-jam } \\
\text { margins against multiple (N-1) } \\
\text { broad-band jammers from any } \\
\text { direction by steering nulls in angle } \\
\text { and main beam towards satellites. }\end{array}$ & \\
\hline
\end{tabular}

Table 2.1: Antenna anti-jamming techniques summary 


\subsection{Conclusion}

In this chapter a basic description of the Global Positioning System (GPS), its operation and history was presented. While GPS applications are growing by the day, its reliability in some areas such as airborne landing and some military guiding systems is still open to question. One of the main disadvantages of GPS is that, because of the high satellite orbits, the signal strength near the earth surface is low, making the receivers susceptible to intentional and unintentional jamming at relatively low power levels. There is a high amount of research and activity all over the world in efforts to increase the interference proofing of the GPS. It is necessary to have an understanding about interference and jamming techniques to be able to anti-jam and protect GPS signals. Some of the most common antenna anti-jamming techniques were presented, which can provide additional improvements to GPS reliability. There are other anti-jamming methods, which can be implemented at the GPS receiver level in the digital domain, and is the subject of many research activities. 


\section{Chapter 3}

\section{MicROSTRIP ANTENNAS}

\subsection{Introduction}

A microstrip patch antenna consists of a very thin metallic patch (usually gold or copper) placed a small fraction of a wavelength above a conducting ground plane, separated by a dielectric substrate. Microstrip antennas have numerous advantages, they are light weight, they can be designed to operate over a large range of frequencies (1$40 \mathrm{GHz}$ ), they can easily be combined to form linear or planar arrays, and they can generate linear, dual, and circular polarizations. These antennas are inexpensive to fabricate using printed circuit board etching, which makes them very useful for integrated active antennas in which circuit functions are integrated with the antenna to produce compact transceivers. Microstrip antennas can be in various shapes and configurations but for the purpose of this project only rectangular microstrip antennas are of interest. This chapter includes an overview of microstrip antennas radiation mechanism, modeling, design, and feeding techniques. The material covered in this chapter known as microstrip antenna theory is from numerous books and articles ([1]-[3], [12]-[24]). 


\subsection{Rectangular Microstrip Antennas}

Rectangular and square patches are the most commonly used type of microstrip antennas. They can be used in numerous types of applications including circular polarization, beam scanning, radiation pattern control and multiple frequency operation. The basic antenna element is a thin conductor of dimensions $\mathrm{L} \times \mathrm{W}$ on a dielectric substrate of permittivity $\varepsilon_{r}$ and thickness $h$ backed by a conducting ground plane. This configuration is shown below:

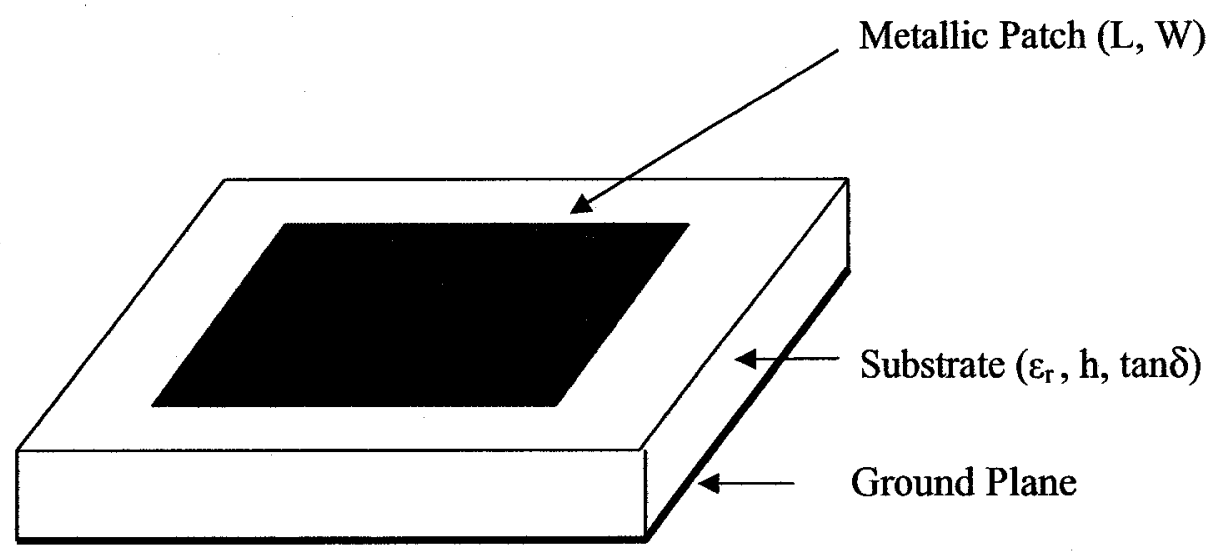

Figure 3.1: Rectangular Microstrip antenna configuration

\subsubsection{Radiation Mechanism}

Radiation from a microstrip antenna is determined from the field distribution between the patch and the ground plane. This can also be described as the surface current distribution on the patch. A patch, which is connected to a microwave source, has a charge distribution on the upper and lower surface of the patch as well as the ground plane. The patch is half wavelength long at the dominant mode, which creates the positive and negative charge distribution shown in Figure 3.2 [11]. 


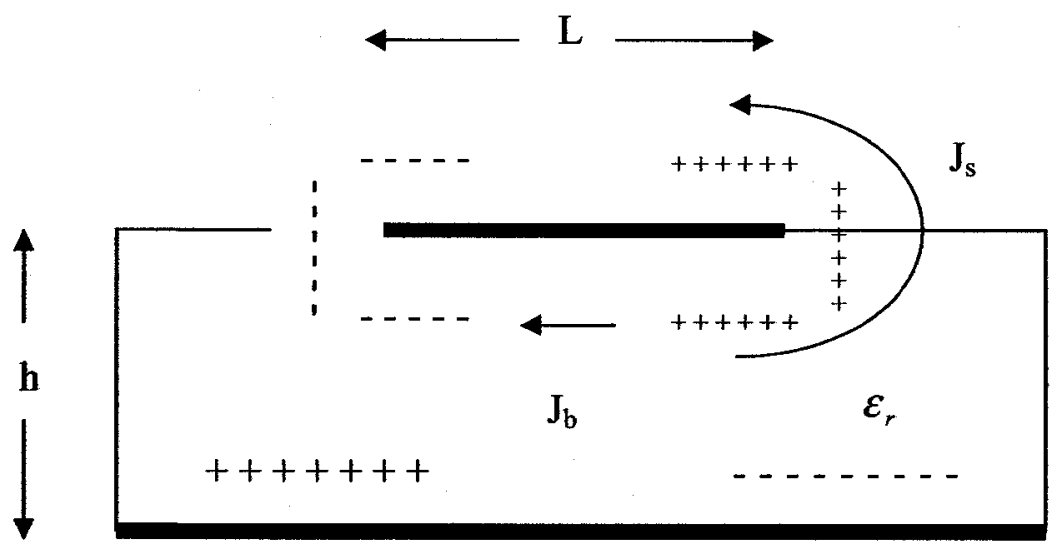

Figure 3.2: Microstrip antenna charge distribution and current density

The repulsive nature of like charges on the bottom surface of the patch, pushes some charges around the side to the top causing current densities $J_{b}$ and $J_{s}$. The ratio $\mathrm{h} / \mathrm{W}$ is small, therefore the strong attractive forces between the charges cause most of the current and charge concentration remains underneath the patch. But also the repulsive force between positive charges creates a large charge density around the edges. The fringing fields caused by these charges are responsible for radiation. In order to achieve better radiation efficiency, thick substrates with lower permittivity are better suited for these types of antennas. Figure 3.3 shows the fringing fields in a microstrip patch [12].

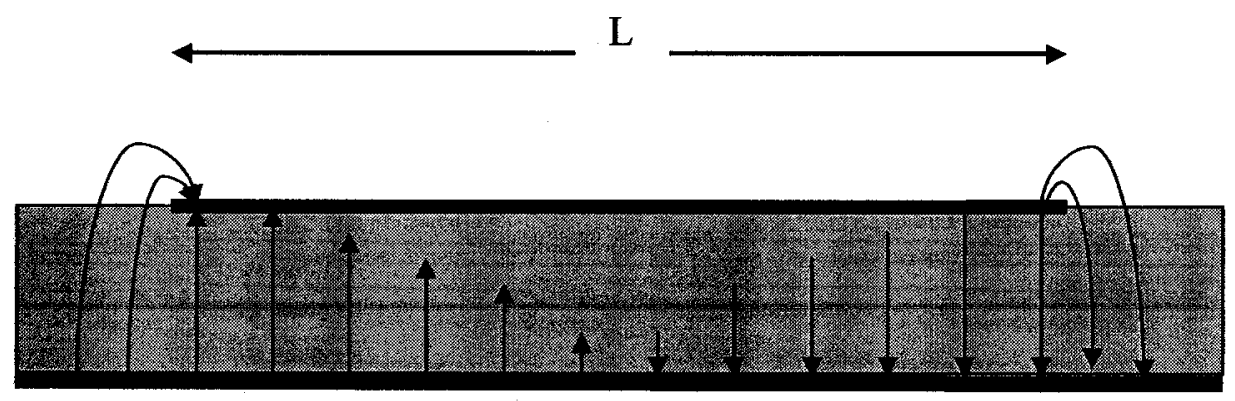

Figure 3.3: Fringing fields for the dominant mode in a rectangular microstrip patch 


\subsection{Microstrip Antenna Analytical Models}

There are various ways to model a microstrip patch. This modeling is used to predict characteristics of a microstrip patch such as resonant frequency, bandwidth, radiation pattern, etc. In this section the transmission line model and the cavity model are presented. These models are based on some assumptions, which simplify the calculations at the cost of less accuracy. There are other models that provide more accuracy such as the full-wave model but are also more complicated to analyze.

\subsubsection{Transmission Line Model}

This is the simplest model and is restricted to rectangular microstrip antennas. This model considers the patch as a transmission line of width $\mathrm{W}$ with two radiating slots on each end. For a desired frequency $f_{0}$, the width $\mathrm{W}$ can be estimated using [12]:

$$
W=\frac{\lambda_{0}}{2} \sqrt{\frac{2}{\varepsilon_{r}+1}}
$$

In this model the input impedance of a patch is the same as that of a transmission line with length $L$ and admittance $Y_{c}$. Each slot has an admittance of $Y_{s}=G_{s}+j B_{s}$ where the values for conductance $G_{s}$ and susceptance $B_{s}$ are given by:

$$
\begin{aligned}
& G_{s}=\frac{W}{120 \lambda_{0}}\left(1-\frac{1}{24}\left(k_{0} h\right)^{2}\right) \\
& B_{s}=\frac{W}{120 \lambda_{0}}\left(1-0.636 \ln \left(k_{0} h\right)\right)
\end{aligned}
$$




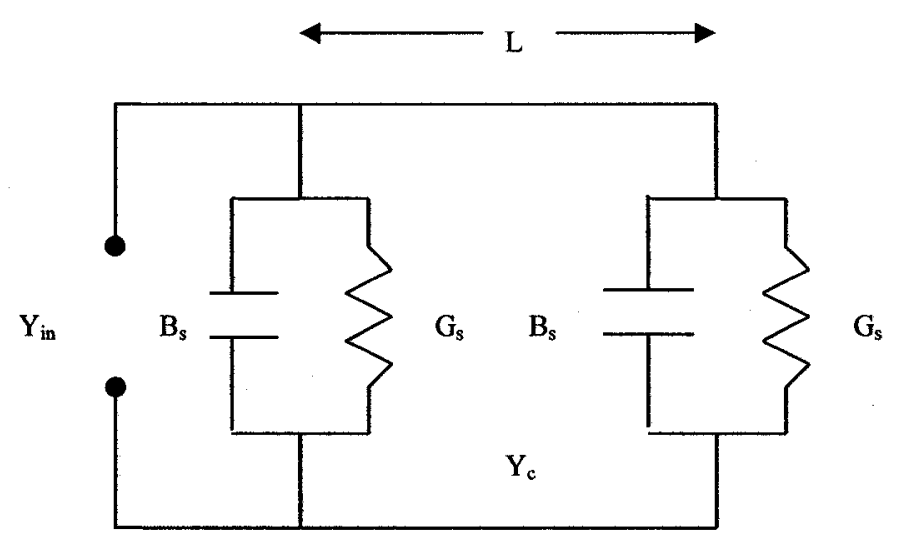

Figure 3.4: Equivalent admittance circuit model

At resonance these slots are $180^{\circ}$ apart, which is achieved by adjusting the length L slightly smaller than half a guided wavelength $\lambda_{\mathrm{g}}$. This adjustment accounts for the fringing fields at the radiating edges so that the susceptance of the two slots cancel each other out, leaving a purely resistive input admittance, so that [12]:

$$
Y_{i n}=2 G_{s}
$$

(Eqn. 3.4)

Therefore at resonance the input resistance at the edge of the patch is:

$$
R_{i n}=\frac{1}{Y_{i n}}
$$

The resistance at an offset distance (s) from the edge is:

$$
R_{i n}=\frac{1}{Y_{i n}} \cos ^{2}\left(\frac{\pi}{L} s\right)
$$


The microstrip patch has an inhomogeneous configuration (air above and dielectric below), which can be replaced by a homogeneous configuration. This is done by introducing a new medium with an effective permittivity $\left(1<\varepsilon_{e f f}<\varepsilon_{r}\right)$, which has the same electrical characteristics (impedance and phase velocity) as the original medium. For $\mathrm{W} / \mathrm{h}>1$, the effective dielectric constant is [12]:

$$
\varepsilon_{\text {eff }}=\frac{\varepsilon_{r}+1}{2}+\frac{\mathcal{E}_{r}-1}{2}\left(1+12 \frac{h}{W}\right)^{-1 / 2}
$$

The waveguide wave length is,

$$
\lambda_{g}=\frac{\lambda_{0}}{\sqrt{\varepsilon_{e f f}}}
$$

The fringing fields around the edges of the patch make it appear electrically larger than its physical length by $2 \Delta \mathrm{L}$. This size increase can be seen in Figure 3.5 .

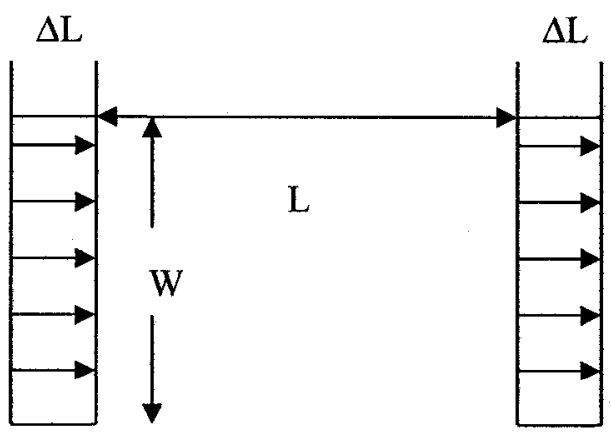

Figure 3.5: Two slot radiation model 
The value $\Delta \mathrm{L}$ is determined from the following:

$$
\Delta L=\frac{0.412 h\left(\varepsilon_{\text {eff }}+0.3\right)\left(\frac{W}{h}+0.264\right)}{\left(\varepsilon_{\text {eff }}-0.258\right)\left(\frac{W}{h}+0.8\right)}
$$

The effective length is given by:

$$
L_{e f f}=(L+2 \Delta L)
$$

The resonant frequency $f_{0}$ (for dominant mode $\mathrm{TM}_{10}$ ) is:

$$
f_{0}=\frac{c}{2 L_{e f f} \sqrt{\varepsilon_{e f f}}}
$$

which can be used to find the value of $L_{e f f}$, and knowing $\Delta \mathrm{L}$ from (Eqn. 3.5), the length $\mathrm{L}$ will be found. The E-fields components of the far field radiation intensity can be found from the following equations [12]:

$$
\begin{aligned}
& E_{\theta}(\theta, \phi)=\frac{-\sin \left(W_{k}\right) \cos \left(L_{k}\right) \cos \phi\left(\varepsilon_{e f f}-\sin ^{2}(\theta)\right.}{W_{k}\left(\varepsilon_{e f f}-\sin ^{2} \theta \cos ^{2} \phi\right)} \\
& E_{\phi}(\theta, \phi)=\frac{\sin \left(W_{k}\right) \cos \left(L_{k}\right) \varepsilon_{e f f} \cos \phi \sin (\theta)}{W_{k}\left(\varepsilon_{e f f}-\sin ^{2} \theta \cos ^{2} \phi\right)}
\end{aligned}
$$


where

$$
\begin{aligned}
& W_{k}=0.5 k W \sin \theta \sin \phi \\
& L_{k}=0.5 k(L+2 \Delta L) \sin \theta \cos \phi
\end{aligned}
$$

where $k$ is the wave number, and the total electric field is:

$$
E(\theta, \phi)=\sqrt{E_{\theta}^{2}(\theta, \phi)+E_{\phi}^{2}(\theta, \phi)}
$$

\subsubsection{Cavity Model}

This model provides a more accurate prediction of input impedance and resonant frequency of a microstrip patch. This technique views the patch as a cavity enclosed by magnetic walls on the sides and electric walls on top and bottom. Due to an electrically thin substrate the fields in the interior region do not vary with $z$. Also inside the cavity the electric field has only a $z$ component $\left(E_{z}\right)$ and the magnetic field has $H_{x}$ and $H_{y}$ components. The electric field is given by the following equation [13]:

$$
E_{z}(x, y)=\sum_{m} \sum_{n} \frac{A_{m n} \chi_{m n}}{\sqrt{\varepsilon_{r} W L h}} \cos \left(k_{x} x\right) \cos \left(k_{y} y\right)
$$

where $A_{m n}$ are the amplitude coefficients corresponding to the electric field mode vectors. Also for the $T M_{m n}$ modes, the resonant wave numbers are given by:

$$
k_{y}=m \pi / L, k_{x}=n \pi / W, k_{m n}^{2}=k_{y}^{2}+k_{x}^{2}
$$


and

$$
\chi_{m n}=\left\{\begin{array}{cc}
1, & m=0 \& n=0 \\
\sqrt{2}, & m=0 \text { or } n=0 \\
2, & m \neq 0 \& n \neq 0
\end{array}\right.
$$

The following figure shows the electric field and magnetic current distributions in walls of a microstrip patch for different modes [1]:

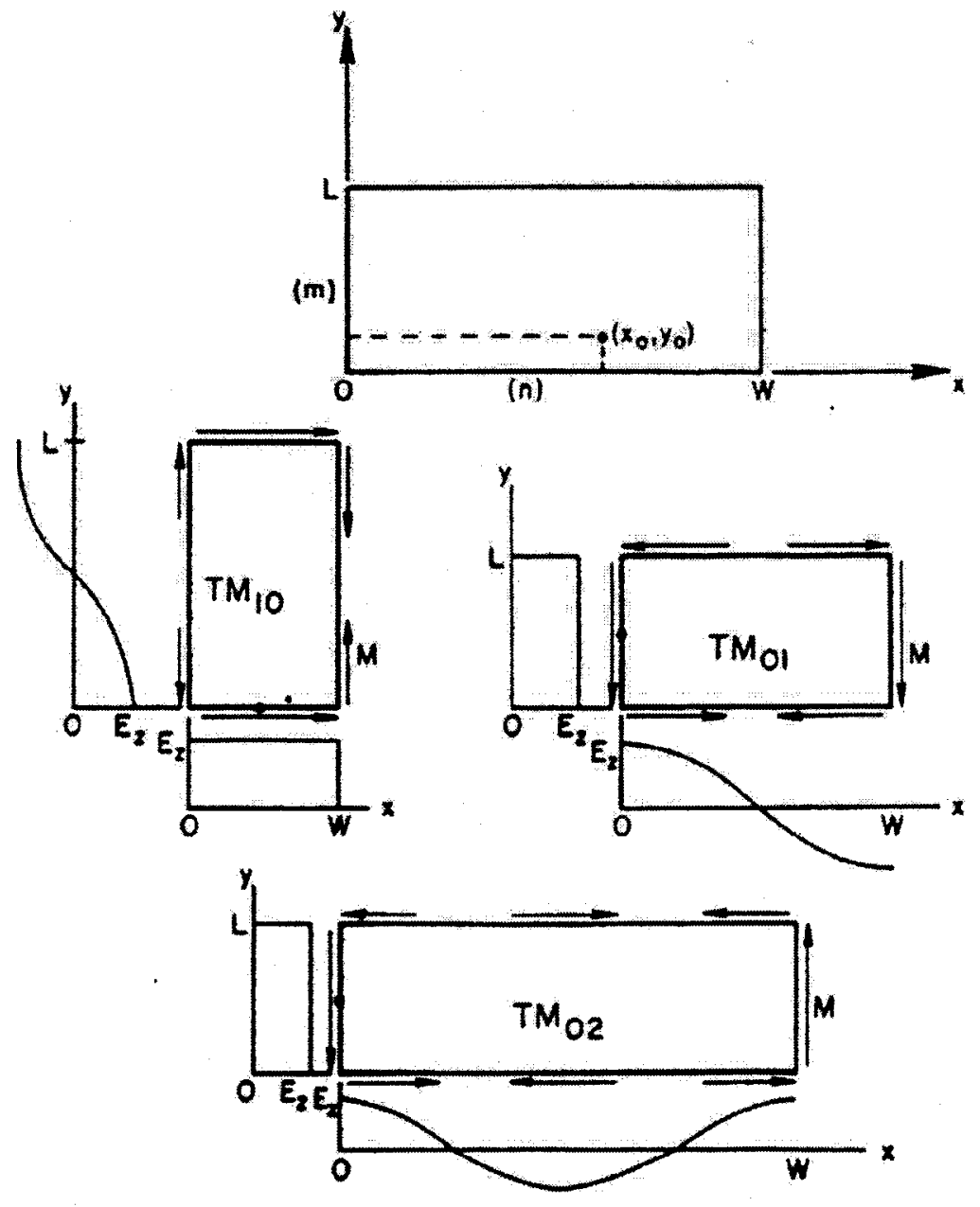

Figure 3.6: Electric field and magnetic current distributions in walls of a microstrip patch for different modes 
It can be seen that for the dominant mode $\mathrm{TM}_{10}$ the electric filed varies only along the length of the patch so that $k_{y}=0$. Therefore in order to estimate the resonant frequency the complex wave number $k_{10}$ must be calculated. The following transcendental equation is obtained [13]:

$$
\tan \left(k_{10} L\right)=\frac{2 k_{10} \alpha_{10}}{k_{10}^{2}-\alpha_{10}^{2}}
$$

where

$$
\begin{gathered}
\alpha_{10}=j \frac{2 \pi Z_{0}}{\lambda_{0}} \frac{h}{W} Y_{w} F_{y}(W / L) \\
F_{y}(W / L)=0.7747+0.5977(W / L-1)-0.1638(W / L-1)^{2}
\end{gathered}
$$

The wall admittance $Y_{w}=G_{w}+j B_{w}$ is multiplied by a factor $F_{y}$ to take into consideration the effect of the aspect ratio $W / L$ on the accuracy of the mode vectors used to represent the actual field distribution at the radiating edges. The wall conductance and susceptance are defined by:

$$
\begin{aligned}
& G_{w}=0.00836 \frac{W}{\lambda_{0}} \\
& B_{w}=0.01668 \Delta L \frac{W}{\lambda_{0}} \varepsilon_{\text {eff }}
\end{aligned}
$$

Where $\varepsilon_{e f f}$ and $\Delta L$ can be found from equations 3.7 and 3.9 respectively. 
An iterative algorithm is suggested to approximate the transcendental equation 3.20. By expanding the first two terms of this equation about $\pi$, such that [14]:

$$
k_{10}=(\pi / L)-\left(\Delta_{4} / L\right)
$$

where

$$
\Delta_{p+1}=\frac{2\left(\alpha_{10} L\right)\left(\pi-\Delta_{p}\right)}{\left(\alpha_{10} L\right)^{2}+2 \Delta_{p} \pi-\Delta_{p}^{2}-\pi^{2}}-\frac{\Delta_{p}^{3}}{3}
$$

and the initial condition $\Delta_{0}=0$. Once $k_{10}$ has been determined the complex radian resonant frequency can be found from the following relation:

$$
\omega=\omega_{\text {real }}+j \omega_{\text {imag }}=\frac{c}{\sqrt{\varepsilon_{r}(1-j \tan \delta)}} k_{10}
$$

where $\tan \delta$ is the loss tangent of the dielectric. The radiation resistance is given by:

$$
R_{10}=\frac{Q_{r}}{\omega_{r} C_{10}}=\frac{2 Q_{r}}{\omega_{r}\left(\varepsilon_{r} L W / h\right)}
$$

where $C_{10}$ is the capacitance referred to patch edge at resonance. The radiation quality factor is given by:

$$
Q_{r}=\frac{\operatorname{Re}\left(k_{10}\right)}{2 \operatorname{Im}\left(k_{10}\right)}
$$




\subsection{Microstrip Antenna Design Considerations}

In order to achieve a desired performance from a rectangular patch, there are some main factors that a designer should consider. This section provides a step-by-step summary for designing an element.

\subsubsection{Geometry}

Choosing a suitable geometry is the first step towards designing a rectangular patch. This is done by selecting a proper substrate and then calculating the element width and length. The substrate permittivity $\varepsilon_{r}$, thickness $h$, and loss tangent $\tan \delta$ selection requires an understanding of the effects they each have on a radiating patch. A thicker substrate provides more strength, increased radiation power, and higher impedance bandwidth. But it is also heavier, and suffers from higher dielectric loss and surface wave loss. The low substrate permittivity $\mathcal{E}_{r}$ has similar effects as a thick substrate; low $\mathcal{E}_{r}$ increases the radiating power by increasing the fringing fields around the edges of the patch. A high loss tangent $\tan \delta$ reduces antenna efficiency by increasing the dielectric loss.

In order to design the antenna element, patch width and length are to be determined. Using equations in section 3.3.1 provides a good approximation of these dimensions. A larger patch width increases the bandwidth and radiation efficiency but has minor effect on the resonant frequency. It is suggested to keep the patch width in the $1<W / L<2$ range. The patch length $\mathrm{L}$ is a more important parameter to find since the resonant frequency is directly dependant on it. 


\subsubsection{Directivity, Gain, and Radiation Efficiency}

Directivity $D$ of an antenna is the measure of energy concentrated in the main beam, which is equal to the ratio of radiation intensity in a given direction compared to an isotropic antenna. A simple estimate for the directivity of a rectangular patch is given by [15]:

$$
D \approx \frac{4\left(k_{0} W\right)^{2}}{\pi \eta_{0} Y_{\text {in }}}
$$

Where $Y_{\text {in }}$ can be calculated from equations (3.2) and (3.4) and $\eta_{0}=120 \pi \Omega$. The directivity is always greater than one since an isotropic antenna is non-directional. The gain $G$ is slightly less than directivity and takes into consideration any losses associated with the antenna. Gain $G$ is related to directivity $D$ by radiation efficiency:

$$
G=e_{r} D
$$

The radiation efficiency is the ratio of the radiated power to the input power and accounts for conduction, dielectric, and surface wave losses associated with the structure. Radiation efficiency can be expressed as [1]:

$$
e_{r}=\frac{P_{r}}{P_{r}+P_{c}+P_{d}+P_{s u r}}
$$


For a low loss substrate powers associated to conduction loss $P_{c}$ and dielectric loss $P_{d}$ are negligible. Radiated and surface wave powers can be obtained from the following equations respectively [16]:

$$
\begin{gathered}
P_{r}=40 k_{0}^{2}\left(k_{0} h\right)^{2}\left(1-\frac{1}{\varepsilon_{r}}+\frac{2}{5 \varepsilon_{r}^{2}}\right) \\
P_{\text {sur }}=30 \pi k_{0}^{2} \frac{\varepsilon_{r}\left(x_{0}^{2}-1\right)}{\varepsilon_{r}\left(\frac{1}{\sqrt{x_{0}^{2}-1}}+\frac{\sqrt{x_{0}^{2}-1}}{\varepsilon_{r}-x_{0}^{2}}\right)+k_{0} h\left(1+\frac{\varepsilon_{r}\left(x_{0}^{2}-1\right)}{\varepsilon_{r}-x_{0}^{2}}\right)}
\end{gathered}
$$

where $x_{0}=\beta / k_{0}$ is the normalized wave number.

\subsubsection{Polarization}

The polarization of an antenna is the same as the polarization of the wave transmitted from it. The electromagnetic wave polarization is the time varying direction and magnitude of the electric-field and generally has an elliptical shape. The ratio of the major axis to the minor axis of the ellipse is known as the axial ratio $(A R)$. A rectangular microstrip patch can be designed to have linear or circular polarization.

In a linear polarized wave the magnitude is the function of time but the direction is constant and therefore $A R=0$ or $\infty$. In this case the $x$ and $y$ components of the electric field have the same phase $\left(\phi_{x}=\phi_{y}\right)$. In the case of a circular polarized wave the $x$ and $y$ components of the electric field have the same magnitude $\left(E_{x}=E_{y}\right)$ and their phases are in quadrature $\left(\phi_{x}-\phi_{y}=\pi / 2\right)$. For the case of clockwise rotation the wave is called 
Right Hand Circular Polarized (RHCP) and for counter-clockwise rotation it is called Left Hand Circular Polarized (LHCP). The GPS signal is RHCP; therefore a RHCP antenna is required to receive the signal properly.

\subsubsection{Radiation Pattern Characteristics}

The radiation pattern in two principal $\mathrm{E}$ and $\mathrm{H}$ planes $\left(\phi=0^{\circ}, \phi=90^{\circ}\right)$ gives enough information about the radiation performance of an antenna. These planes are always orthogonal and contain the field vector and the direction of maximum radiation. Generally the radiation pattern of an antenna contains several lobes. The lobe with maximum directivity is known as the main lobe and the rest are considered side lobes or minor lobes. An antenna radiation pattern contains information such as directivity, beam width, side lobe, and back lobe levels. Beam width can be defined in a number of ways such as half-power beam width (HPBW) and beam width between first nulls (FNBW). Maximum Side Lobe Level is also a ratio that should be kept as high as possible. It is also desirable in most cases to minimize the back lobe radiation, so the Front to Back Ratio (FBR) should be also as high as possible. Figure 3.7 shows these relations:

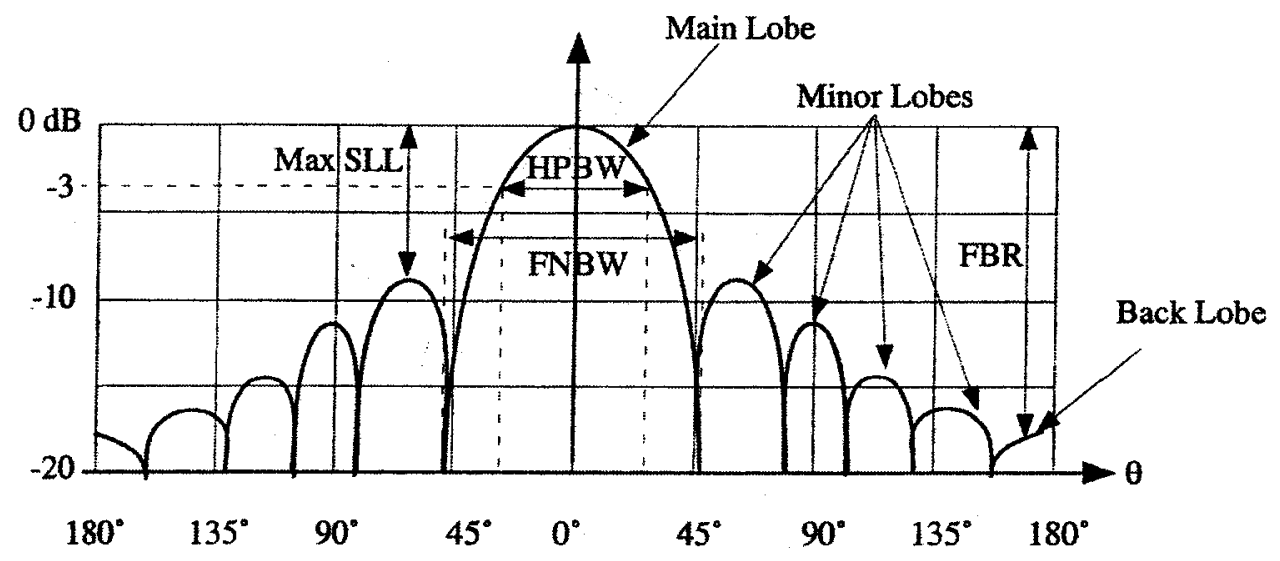

Figure 3.7: Antenna Pattern Characteristics [12] 


\subsubsection{Bandwidth}

The bandwidth of an antenna is the range of frequencies in which the antenna has satisfactory operation. Antenna bandwidth can be defined in different ways depending on the characteristic selected. Since different characteristics such as input impedance, gain, polarization (AR) and beamwidth all change differently with frequency there is no unique way to define an antenna bandwidth. The impedance bandwidth is the range of frequencies in which a suitable impedance match is achieved. For dielectrics with low permittivity the following equations provide a good approximation for impedance bandwidth [17]:

$$
B W=\frac{16}{3 \sqrt{2}} \frac{p}{e_{r}} \frac{1}{\varepsilon_{r}} \frac{h}{\lambda_{0}} \frac{W}{L} q
$$

where

$$
\begin{gathered}
p=1-\frac{0.16605}{20}\left(k_{o} W\right)^{2}+\frac{0.02283}{560}\left(k_{o} W\right)^{4}-0.009142\left(k_{o} L\right)^{2} \\
q=1-\frac{1}{\varepsilon_{r}}+\frac{2}{5 \varepsilon_{r}^{2}}
\end{gathered}
$$

A microstrip patch can be modeled as a high quality $(Q)$ factor RLC circuit near its resonant frequency. This $Q$ factor can be used to determine the bandwidth of the antenna using:

$$
B W=\frac{S-1}{Q_{r} \sqrt{S}}
$$


where $\mathrm{S}$ is the maximum desired VSWR $(\mathrm{VSWR}<\mathrm{S})$ and radiation quality factor is:

$$
Q_{r}=\frac{2 \omega W_{T}}{P_{r}}
$$

where radiated power $P_{r}$ is given by equation $3.33 . W_{T}$ is the energy stored at resonance and is given by:

$$
W_{T}=\frac{1}{4} \varepsilon_{0} \varepsilon_{r} h L W
$$

The bandwidth of a microstrip patch is usually 1-5\% depending on the quality of the matching network. There are several techniques for increasing a microstrip antenna's bandwidth. Increasing the substrate thickness $h$ results in a lower $Q$ factor, therefore larger bandwidth. But there are limitations for this thickness due to excitation of undesirable surface waves. The surface wave loss can be neglected for a value of $h$ satisfying the following equation [18]:

$$
h \leq \frac{0.3 \lambda_{0}}{2 \pi \sqrt{\varepsilon_{r}}}
$$

Larger bandwidth can also be achieved by choosing a dielectric with lower permittivity $\left(Q \propto \sqrt{\varepsilon_{r}}\right)$. This lower permittivity results in a larger patch since $L \propto 1 / \sqrt{\varepsilon_{r}}$, which is unwanted where there is a size limitation. 


\subsubsection{Effect of Finite Size Ground Plane:}

The analysis and design of a microstrip antenna assumes an infinite ground plane. It is not feasible to achieve such a thing, also due to limitations in size of an antenna it is necessary to have a finite ground plane. The finite size ground plane changes the radiation pattern, resonant frequency, radiation conductance, and gain. These changes can be explained to be caused by the diffraction of radiation from the edges of the ground plane. Results of experimental investigations show that ground plane size is inversely proportional to the resonant frequency. The following graph shows this change in resonant frequency [19]:

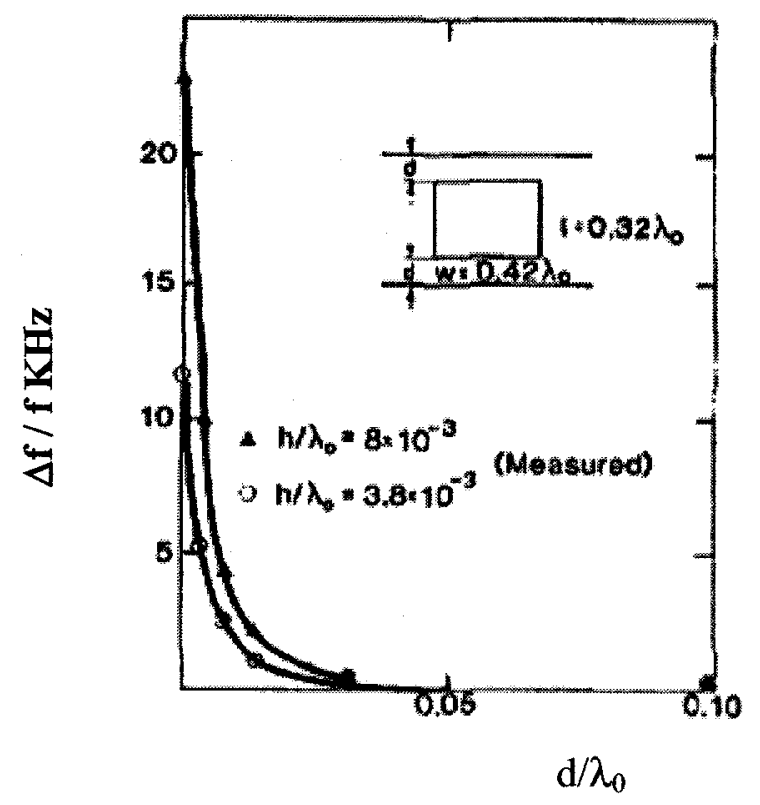

Figure 3.8: Change in resonant frequency vs. ground plane size [19]

As mentioned before, the finite ground plane creates changes in the radiation pattern. This change is more visible in the E-field plane than in the H-field plane. As the size of the ground plane is increased, ripples in the main beam appear along with scattered radiation in the back lobes. This can be seen in the following figure [20]: 


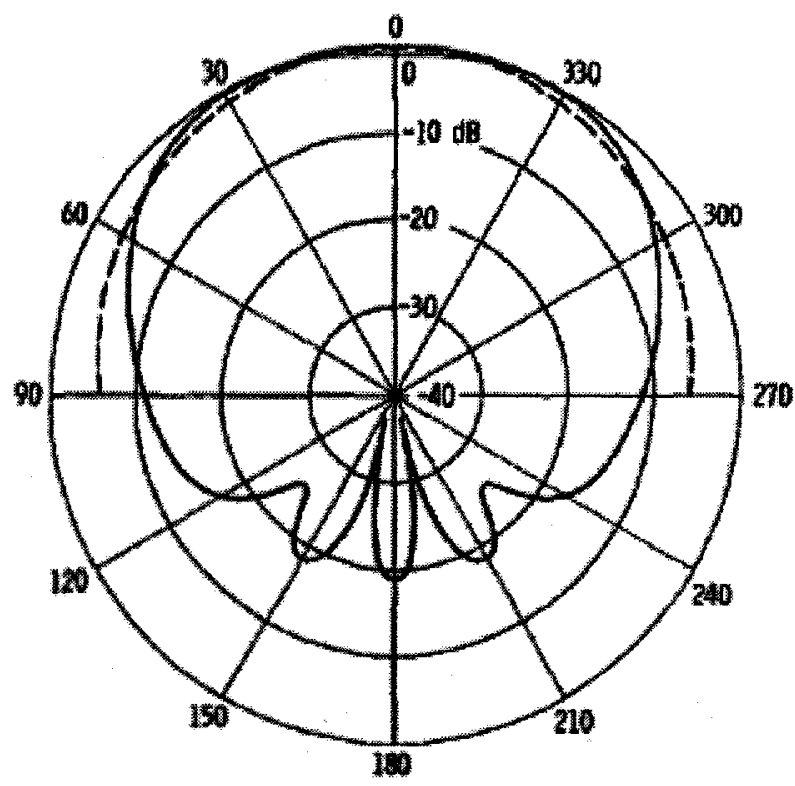

Figure 3.9: E-plane pattern for infinite vs. finite ground plane [20] $2 \lambda \mathrm{GP}$ Infinite GP

\subsection{Microstrip Antenna Feeding Techniques:}

In this section some of the most common feeding techniques are presented. These techniques are discussed in details using the mechanism of coupling energy and equivalent circuits. Impedance matching is the most important aspect in feeding an antenna no matter what technique is being used. This matching assures that most of the power is transferred from the feed to the radiating patch. But also associated with impedance matching are spurious radiation and surface wave losses, which are caused by discontinuities such as bends, junctions, stubs, and stepped impedance transformers. It is also important to consider the suitability of a feed for antenna arrays. In this section the coaxial probe feed, the coplanar microstrip line feed, and the aperture-coupled feed are discussed. Aperture-coupled feeding is discussed in more detail since it is the technique used in this design. 


\subsubsection{Coaxial Probe Feeding}

One of the basic mechanisms of transferring power to a microstrip patch is by using a probe passed through the substrate and soldered to the patch. This probe can be the inner conductor of the coaxial line or a strip line through a slot in the ground plane. The location of the slot should be at the point where the best impedance match is achieved. The coupling of the feed current $J_{z}$ to the $E_{z}$ field results in excitation in the patch. The coupling can be obtained by the following equation [21]:

$$
\text { Coupling }=\iiint_{V} E_{z} J_{z} d \nu \cong \cos \left(\pi x_{0} / L\right)
$$

where $x_{0}$ is the slot offset from the edge of the patch. It can be seen that the coupling is maximum at the edge of the patch. The following figure shows the top and side views of an N-type coaxial connector:
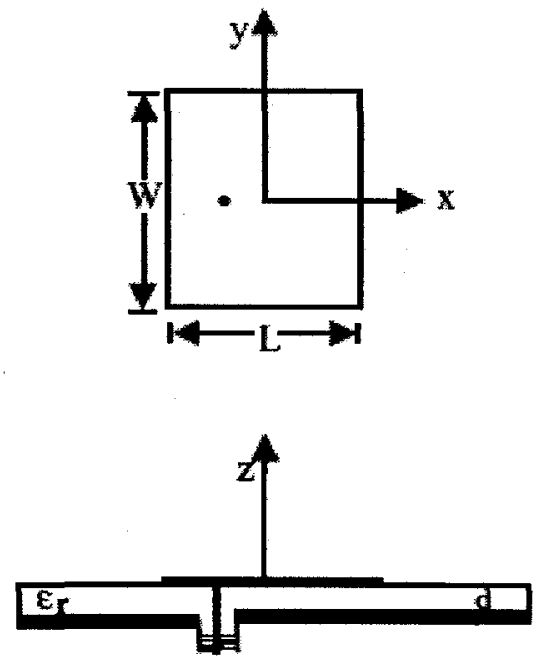

Figure 3.10: Coaxial probe fed microstrip patch antenna [20] 
The main advantage of the probe feeding technique is that the input impedance level can be easily adjusted and positioning of the feed is quite simple. There are limitations associated with this kind of feeding. First, probe feeding is not suitable for arrays since a large amount of soldering will be required, which is inefficient. Also for thick substrates a long probe is required, which causes additional spurious radiation, feed inductance and surface wave.

\subsubsection{Coplanar Microstrip Line Feeding}

Feeding a microstrip patch with a microstrip transmission line on the same layer is the most common choice for antenna designers. The microstrip patch can be considered to be the extension of the microstrip line and can be fabricated at the same time as the patch. Similar to the probe feed, the excitation is a result of the coupling between the feed current $J_{x}$ to the $E_{z}$ field and can be found using equation 3.42. Microstrip feeding can be used in various ways that are shown in Figure 3.11.

This feeding technique is easy to design and fabricate but also suffers from spurious radiation. There are also limitations with each configuration. The edge-coupled feed required additional matching network since the impedance at the patch edge is higher than $50 \Omega$. This matching circuitry increases the spurious radiation and also makes

the design more difficult, especially for arrays with limited space. The gap-coupled feed requires a very precise gap width for efficient coupling. Also the open end of the microstrip line increases the spurious radiation. The inset feeding overcomes some of these issues such as $50 \Omega$ matching network but suffers from a decrease in patch radiation. 


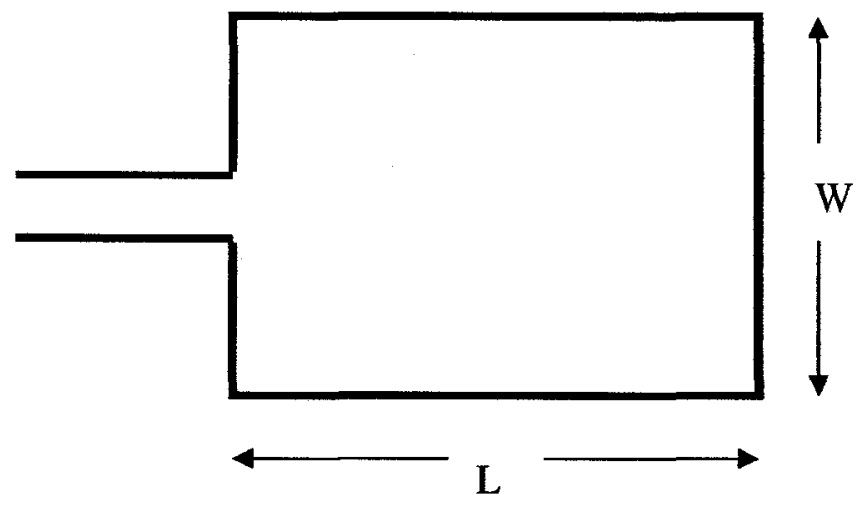

(a)

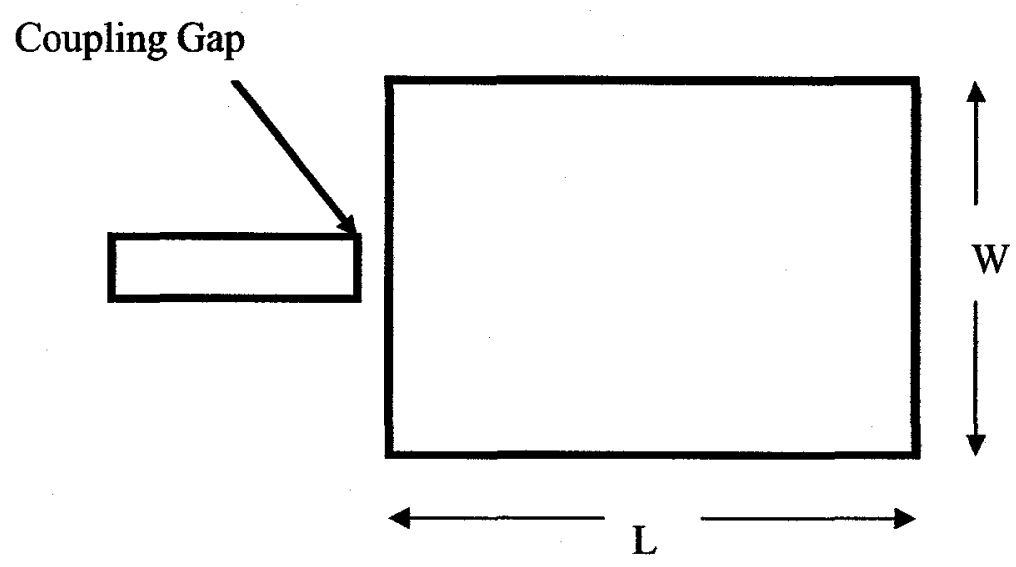

(b)

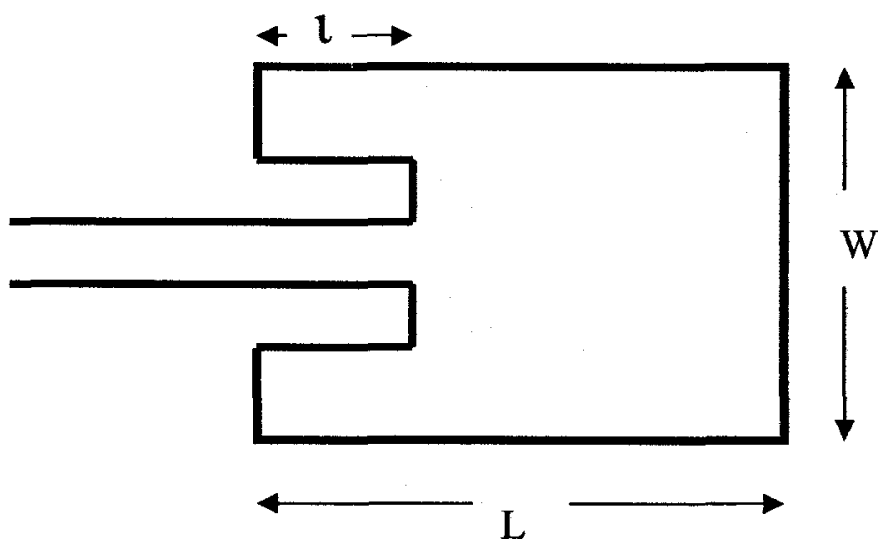

(c)

Figure 3.11: Coplanar microstrip feeding (a) feed at edge

(b) gap-coupled feed (c) inset feed at edge 


\subsubsection{Aperture-coupled Feeding}

In this technique the feed network is separated from the radiating patch by a common ground plane. Energy is electromagnetically coupled through an aperture in the ground plane. This aperture is usually centered with respect to the patch where the patch has its maximum magnetic field. For maximum coupling it has been suggested that a rectangular slot parallel to the two radiating edges should be used. Two very similar coupling mechanisms take place, one between the feed line and the slot and another between the slot and the patch. The coupling amplitude can be obtained by the following equation [21]:

$$
\text { Coupling }=\iiint_{V} \bar{M} \cdot \bar{H} d v \cong \sin \left(\pi x_{0} / L\right)
$$

In this equation $x_{0}$ is the offset of the slot from the patch edge. This technique has several advantages, which makes it suitable for widespread applications in communication systems. Also, the isolation of the feed network from the patch, reduces the spurious radiations and provides more space for the feed network and is suitable for phased arrays. Wider bandwidth can be achieved by adjusting the width and length of the coupling slot and using a thicker patch substrate. The feed substrate is usually thin with high permittivity, whereas the patch substrate can be thick with low permittivity. The geometry of a basic aperture-coupled microstrip antenna is shown in Figure 3.12 [22]: 


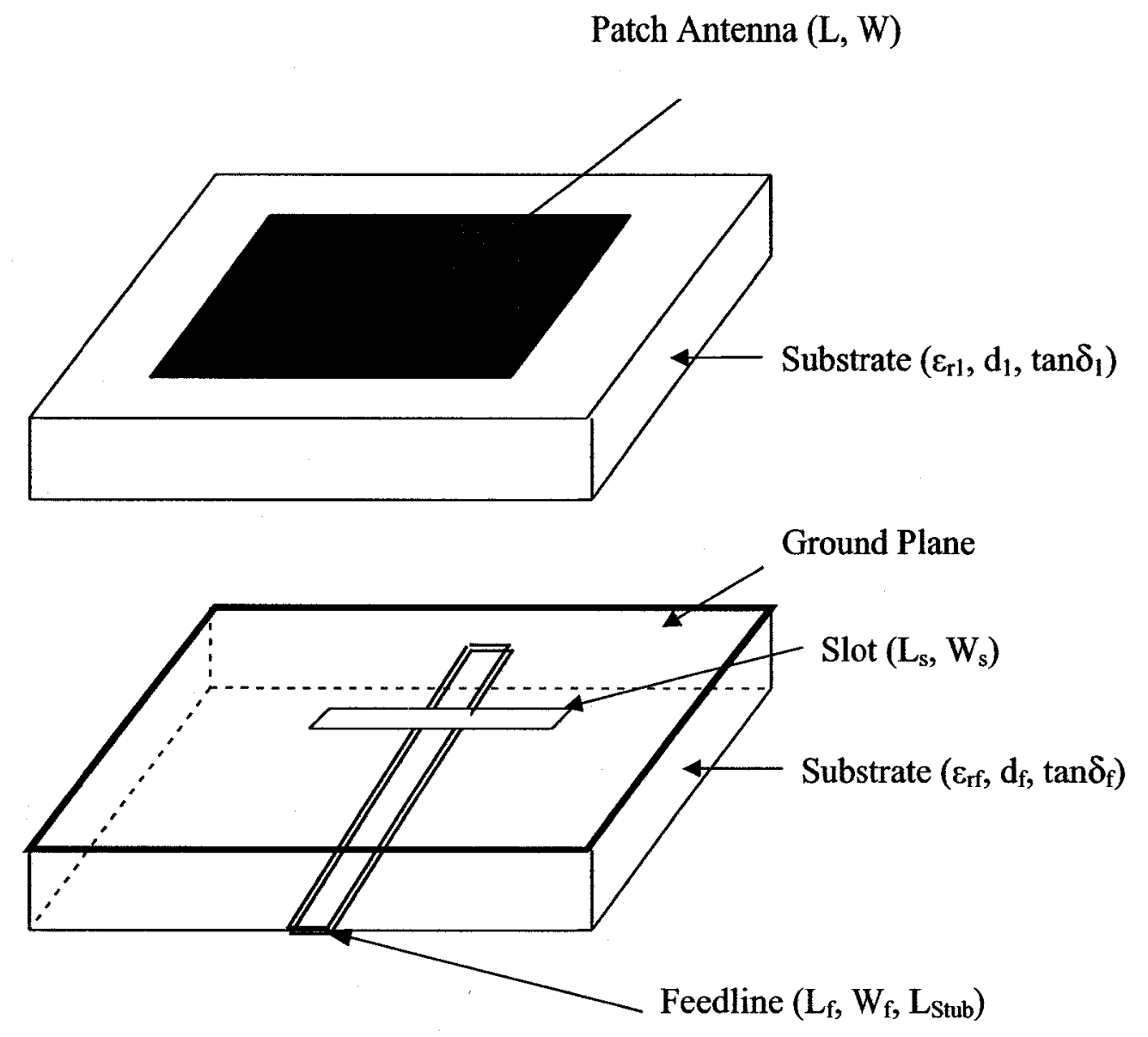

Figure 3.12: Aperture-coupled feeding technique

There are several methods to analyze aperture-coupled microstrip antennas such as the integral equation approach, cavity model, transmission line model, model expansion method, and a hybrid approach. Here, we discuss the transmission line model because of its ability to arrive at a simpler design. 
A simplified equivalent circuit of a slot coupled microstrip patch antenna is shown below [23]. The microstrip patch is characterized by admittance $Y_{\text {patch }}$ and the aperture by admittance $\mathrm{Y}_{\mathrm{ap}}$ :

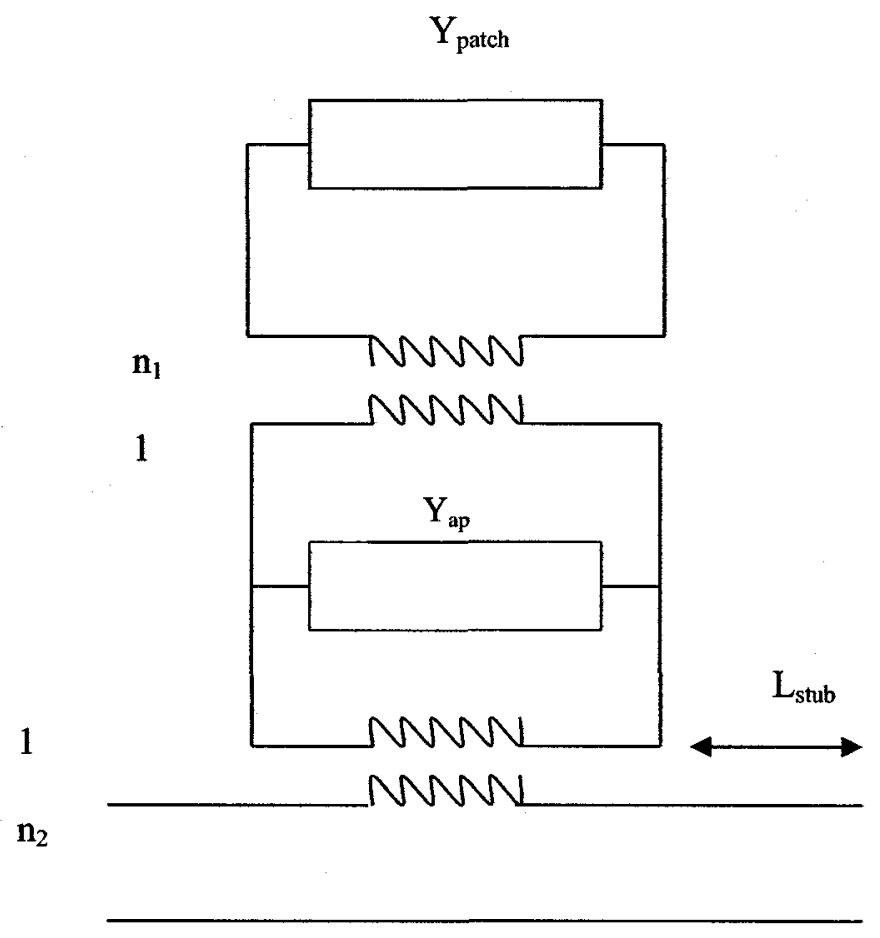

Figure 3.13: Simplified circuit of aperture-coupled patch

The slot interrupts the longitudinal current flow in the feed line and the patch, resulting in a coupling between them. The coupling between the patch, aperture, and the feed line can be described by two impedance transformers. The $1: n_{1}$ transformer has a turns ratio approximately equal to the fraction of patch current intercepted by the aperture to the total patch current, so that [24]:

$$
n_{1}=L_{s} / W
$$


and,

$$
\begin{aligned}
n_{2}= & \frac{J_{0}\left(k_{e s} W_{f} / 2\right) J_{0}\left(k_{e m} W_{s} / 2\right)}{k_{e s}{ }^{2}+k_{e m}{ }^{2}} \\
& {\left[\frac{k_{e m}{ }^{2} k_{2} \varepsilon_{r f}}{k_{2} \varepsilon_{r f} \cos k_{1} d_{p}-k_{1} \sin k_{1} d_{p}}+\frac{k_{e s}{ }^{2} k_{1}}{k_{2} \cos k_{1} d_{p}+k_{1} \sin k_{1} d_{p}}\right] }
\end{aligned}
$$

where $J_{0}(\bullet)$ is the zeroth-order Bessel function and

$$
\begin{aligned}
& k_{1}=k_{0} \sqrt{\left|\varepsilon_{r f}-\varepsilon_{e f f s}-\varepsilon_{e f f m}\right|} \\
& k_{1}=k_{0} \sqrt{\left|\varepsilon_{e f f s}+\varepsilon_{e f f m}-1\right|} \\
& k_{e s}=k_{0} \sqrt{\varepsilon_{e f f s}} \quad, k_{e m}=k_{0} \sqrt{\varepsilon_{e f f m}}
\end{aligned}
$$

The transmission line equivalent circuit of microstrip patch is shown below [23]:

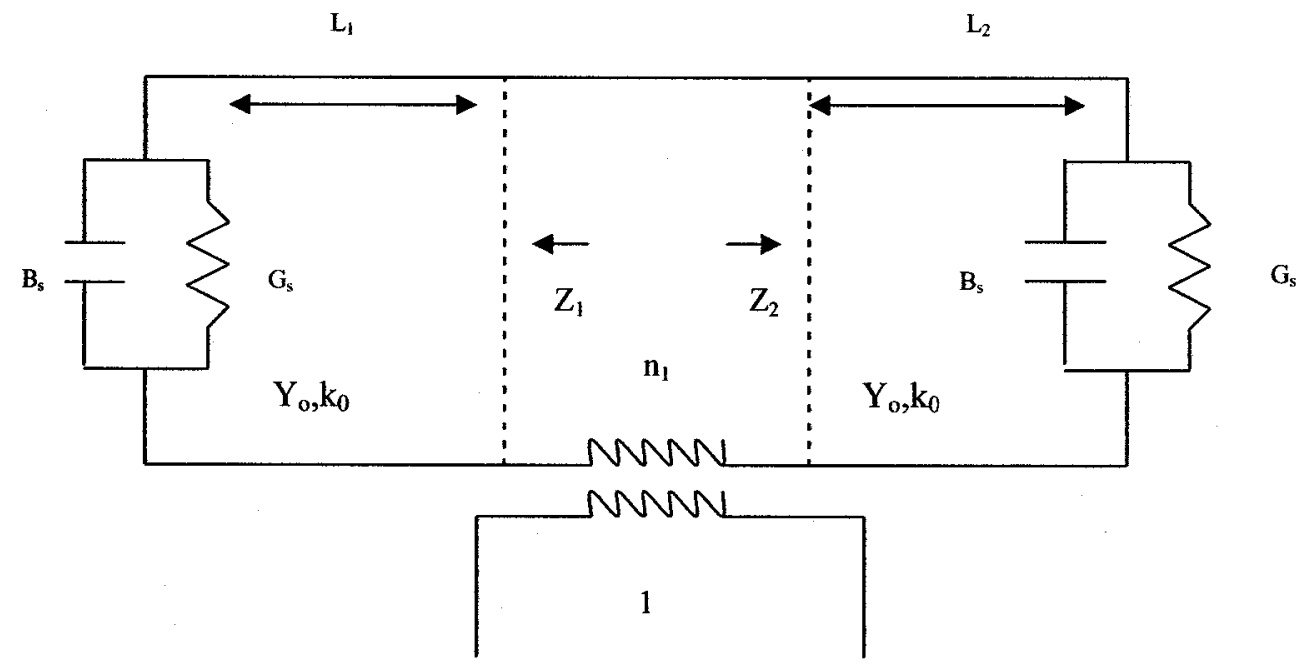

Figure 3.14: Transmission line equivalent circuit of microstrip patch 
In this circuit $Z_{1}$ and $Z_{2}$ are impedances looking to the left and right of the aperture. The microstrip line has an impedance of $Z_{0 \mathrm{~m}}$ and the slot has an impedance of $Z_{0 s}$. The patch impedance is given by:

$$
Z_{\text {parch }}=Z_{1}+Z_{2}=1 / Y_{1}+1 / Y_{2}
$$

where

$$
\begin{array}{ll}
Y_{1}=Y_{0} \frac{\left(G_{r}+j B_{\text {open }}\right)+j Y_{0} \tan \left(k_{0} L_{1}\right)}{Y_{0}+j\left(G_{r}+j B_{\text {open }}\right) \tan \left(k_{0} L_{1}\right)} & L_{1}=x_{0} \\
Y_{2}=Y_{0} \frac{\left(G_{r}+j B_{\text {open }}\right)+j Y_{0} \tan \left(k_{0} L_{2}\right)}{Y_{0}+j\left(G_{r}+j B_{\text {open }}\right) \tan \left(k_{0} L_{2}\right)} & L_{2}=L_{\text {pach }}-L_{1}
\end{array}
$$

In these equations $\left(G_{r}+j B_{\text {open }}\right)$ is the edge admittance of the patch and $\left(Y_{0}, k_{0}\right)$ characterize the patch as a transmission line of width $W$. Since the slot is electrically small the aperture admittance $Y_{a p}$ is inductive. $Y_{a p}$ explains the energy stored near the slot and is given by:

$$
Y_{a p}=-2 j Y_{0 s} \cot \left(k_{0} L_{s} / 2\right)
$$

The input impedance of the antenna at the center of the aperture is given by:

$$
Z_{\text {in }}=\frac{n_{2}^{2}}{n_{1}^{2} Y_{p a t c h}+Y_{a p}}-j Z_{0 m} \cot \left(k_{e m} L_{S u b}\right)
$$


and the condition for resonance is given by:

$$
B_{\text {patch }} \approx \frac{4 W^{2}}{Z_{0 s} k_{e s} L_{s}{ }^{3}}
$$

This shows that by increasing $L_{s}$ the resonant frequency decreases. The transmission line model can be extended to include different aperture shapes such as cross, $\mathrm{H}$, etc. It has been recommended to follow the following rules of thumb in choosing the slot width and length [22]:

$$
\begin{gathered}
L_{s}>W_{f}+n d_{f} \quad \text { with } n>6 \\
W_{s} / L_{s}=1 / 10
\end{gathered}
$$

\subsection{Conclusion:}

This chapter has presented an overview of rectangular microstrip antennas. It was shown that the fringing fields caused by a charge distribution on the upper and lower surface of the patch are responsible for radiation. In order to achieve better radiation efficiency, thick substrates with lower permittivity are better choice for these types of antennas. In this section the transmission line and the cavity models were presented, which are the two simplest modeling techniques for microstrip antennas. For more accurate models such as the full-wave analysis model, one can refer to many published 
resources [1]. Various design considerations such as geometry, gain, polarization, bandwidth, efficiency, and ground plane effect were presented. Different feeding techniques were also presented in this section and aperture-coupled feed was discussed in details. It is important for an antenna designer to reach to an optimum configuration to satisfy the design requirements. It is not always possible to achieve the perfect design since there is a trade off between antenna characteristics. 


\section{Chapter 4}

\section{Stacked Microstrip Antenna Design}

\subsection{Introduction}

Stacked microstrip patches can be used to achieve dual or multiple frequency operation. Also it has been shown that stacking microstrip patches result in an increased impedance bandwidth over a single layer radiator. In this chapter a dual-patch configuration will be studied. In this configuration two microstrip patches with slightly different sizes are stacked on top of each other on a common ground plane. These two patches can be fed separately in a piggyback configuration, or together by the means of an aperture-coupled feeding, probe feeding, or microstrip line feeding. The upper patch is coupled to the excited lower patch and may radiate at a second resonant frequency. In this configuration each patch can use a thin substrate layer to maintain a low profile design and to minimize the excitation of surface waves. Also the designer can take advantage of several extra degrees of freedom such as element offset, size, and substrate thicknesses. The aperture-coupled configuration has an additional advantage; it isolates spurious feed radiation by the use of the common ground plane. 
This chapter describes a summary of relevant studies on aperture-coupled stacked patch antennas along with the radiation mechanism and characteristics of this configuration. Also the design procedure of a dual-band aperture-coupled stacked patch antenna in GPS frequencies L1 and L2/L5 is included. Two similar antenna structures were designed using different substrate material. Since the GPS signal is right hand circularly polarized it is required for the designed antennas to be also right hand circular polarized. An exploded view of a multilayered linear polarized aperture-coupled stacked microstrip antenna is shown in Figure 4.1:

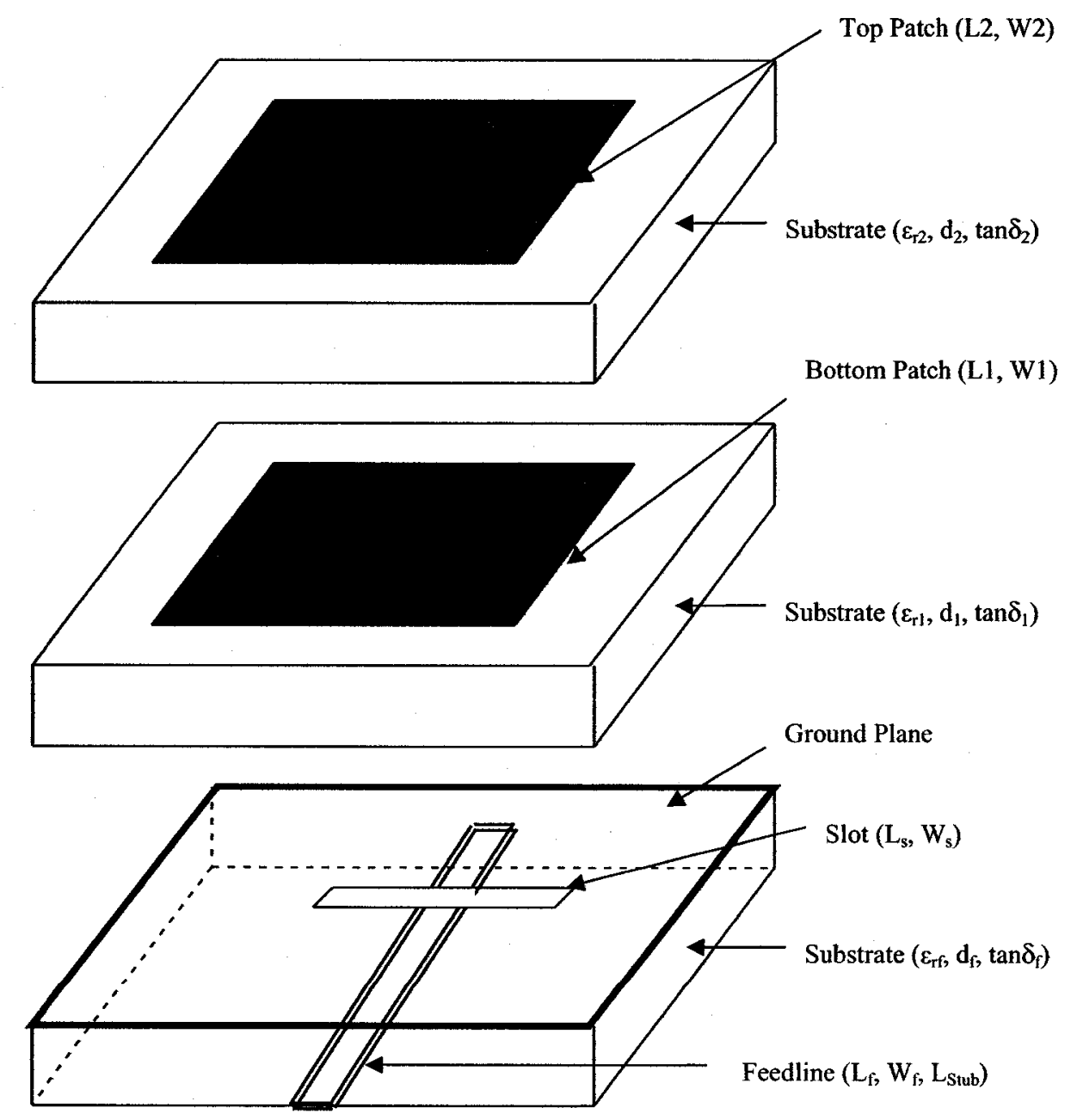

Figure 4.1: Aperture-Coupled Stacked Microstrip Antenna Exploded Geometry 


\subsection{Coupling Analysis}

In order to analyze the coupling between elements, the electric current distribution on the patches or the field distribution between them can be studied. Two modes of operation are possible depending on the currents phase. The in-phase currents, known as the even mode, happen at a lower frequency and are suitable for radiation; while the outof-phase currents, known as the odd mode, happen at a higher frequency and are not suitable for radiation. The electric and magnetic field distribution for the even mode of operation is shown in the following figure [1]. The patches are coupled through the magnetic field.

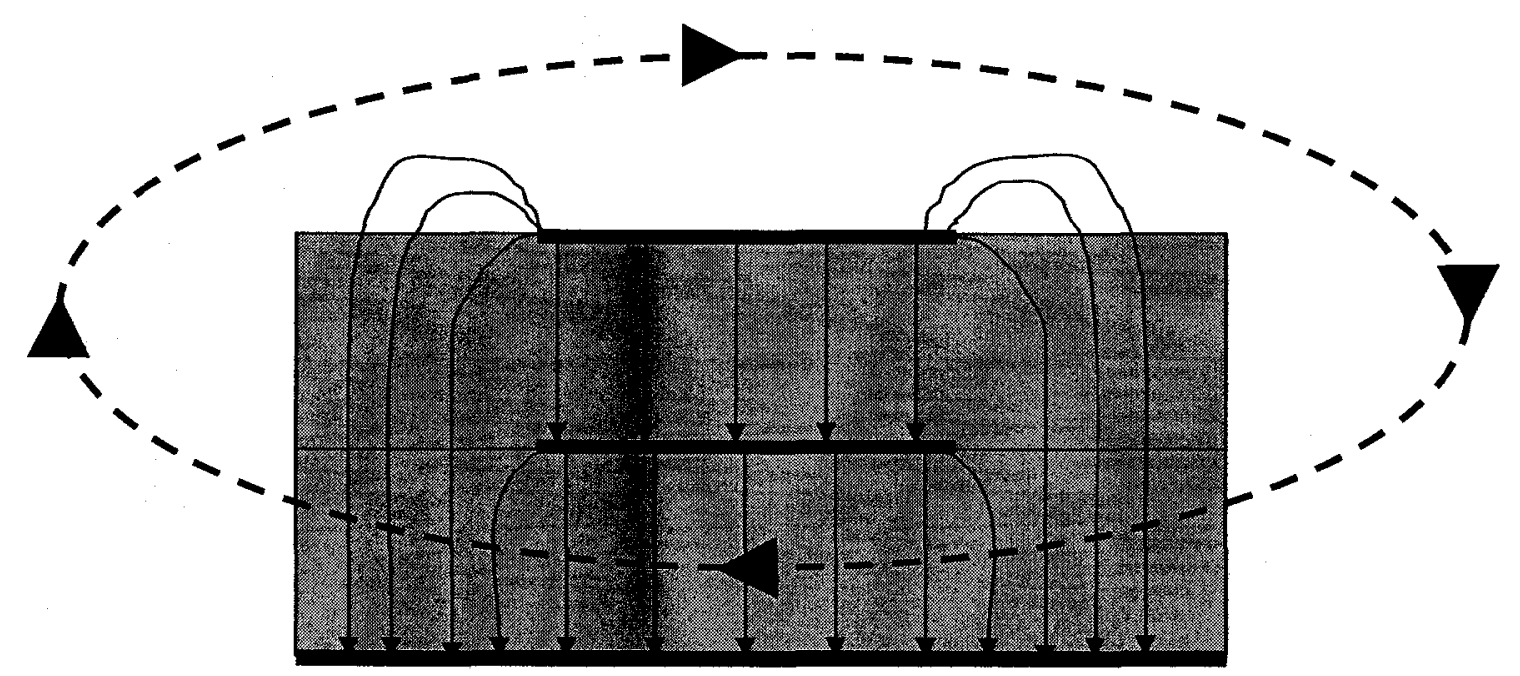

Figure 4.2: Electric and magnetic field distribution for the even mode

--- Magnetic Field _ Electric Field 


\subsection{Studies on Aperture-Coupled Stacked Patch Antennas}

Targonski, Waterhouse and Pozar [7] studied two different impedance matching configurations on a linear polarized stacked patch. In this design an aperture larger and a substrate thicker than similar configurations were used. The two feedline configurations are shown in Figure 4.3:

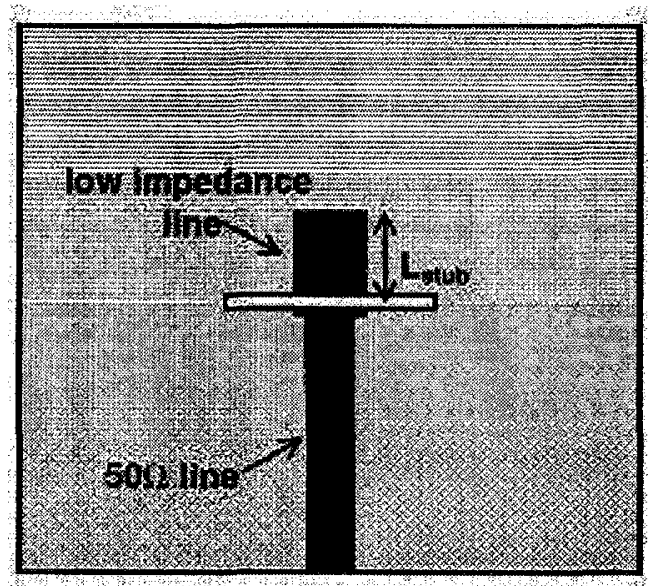

(a)

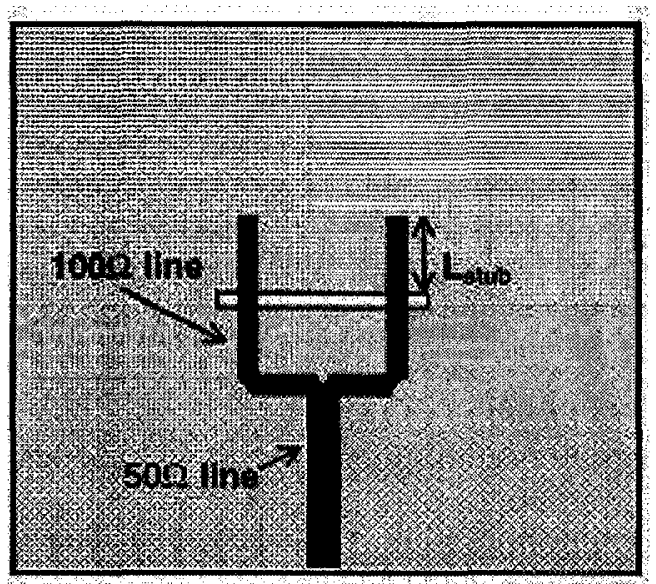

(b)

Figure 4.3: (a) Wide-centered feedline. (b) Dual-offset feedline [7]

There is a high level of coupling between the feedline and the aperture, which must be reduced to achieve proper matching. The wide-centered feedline achieves this goal, since by thickening the feedline the coupling decreases. The dual-offset feedline uses two $100 \Omega$ lines, which are joined by a reactive power combiner. This technique takes advantage of a lower cross-polarization level compare to the wide-centered feedline. An impedance bandwidth of $25 \%$ was achieved by these techniques. 
Another work by Targonski and Pozar [6] describes the design of a wideband circular polarized aperture-coupled microstrip antenna. In this study, parallel and series feeding techniques were described and compared. In order to achieve circular polarization a cross aperture was used, by which two orthogonal linear polarized modes were independently excited with equal amplitudes and a $90^{\circ}$ phase shift. These two feeding configurations are shown in Figure 4.4:

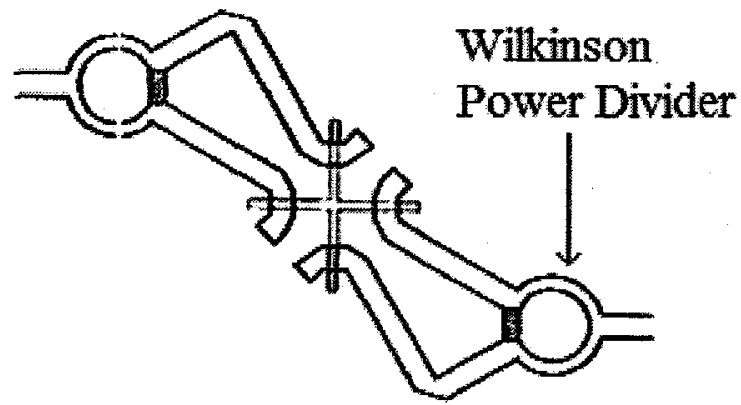

(a)

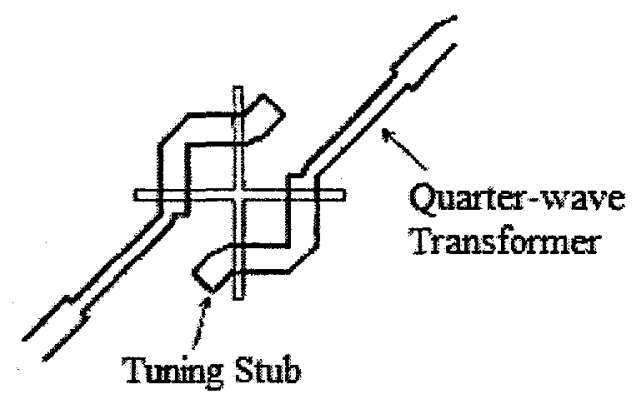

(b)

Figure 4.4: (a) Parallel feed configuration. (b)Series feed configuration.[6]

Each port is fed $180^{\circ}$ out of phase. In the parallel feed configuration the $90^{\circ}$ phase shift was produced by extending one arm of the output feed line by a quarter of a wavelength. In the series feed configuration a quarter wavelength section of transmission line is placed between each aperture. It was shown that the parallel feed configuration provides better bandwidth and axial ratio; but is also more complicated to design because of the Wilkinson power dividers. In this design an impedance bandwidth of $22 \%$ was achieved. A similar work by Pozar and Duffy [5] addresses the design of an aperturecoupled stacked patch antenna using the parallel feed configuration. 


\subsection{Antenna Design}

This section addresses the design procedure of a dual-band aperture-coupled stacked patch antenna in GPS frequencies L1 and L2/L5. Two similar antenna structures were designed using different substrate material. The first antenna was designed so that the fabrication and material cost was minimal. The reason for designing a second antenna was to reduce the dimensions of the first antenna at the cost of more expensive material with higher permittivity.

These antennas were designed so that the first band located at $\mathrm{L} 1=1575.42 \mathrm{MHz}$ has an impedance bandwidth of $20 \mathrm{MHz}$ to $50 \mathrm{MHz}(2-4 \%)$; and the second band located at $\mathrm{L} 2=1227.60 \mathrm{MHz}$ and $\mathrm{L} 5=1176.45 \mathrm{MHz}$ is wide enough to cover both frequencies and has an impedance bandwidth of $70 \mathrm{MHz}$ to $150 \mathrm{MHz}(6-12.5 \%)$. It is also desirable to achieve a high rejection level at other frequency bands. These antennas are also circularly polarized (RHCP) so the axial ratio bandwidth $(\mathrm{AR}<3 \mathrm{~dB})$ should be enough to cover all desired frequency bands. There is no step-by-step design procedure for this kind of structure due to the number of interacting design parameters. The following design is a combination of derivations, empirical tuning and computer modeling to arrive at a functional design that meets the above requirements.

\subsubsection{Selected Geometry:}

At first it is necessary to distinguish the design parameters and the effects they have on resonant frequency, bandwidth, and coupling. These design parameters and their effects are listed [5]: 
1. Bottom patch substrate thickness and permittivity: Coupling to the aperture decreases with substrate thickness; therefore the aperture size should be increased, which causes back lobe radiation. A thick substrate with low permittivity results in larger bandwidth.

2. Bottom patch size: For circular polarization, two orthogonal linear polarizations with equal amplitude and a $90^{\circ}$ phase shift must be generated; therefore the patches must be square. Because of a strong coupling between patches, patch sizes cannot be determined independently and it is the relative patch size that matters.

3. Top patch substrate thickness and permittivity: Substrate thickness and permittivity of the top patch control the coupling to the bottom patch along with the bandwidth of the associated band.

4. Top patch size: The top patch is also square and is excited by coupling to the bottom patch. This coupling is dependant on the relative size of the patches and also whether the top patch is smaller or larger than the bottom patch. The relative patch sizes must be adjusted for proper impedance match. Since the lower frequency band L2/L5 requires larger bandwidth than the L1 band the top patch is chosen to be larger. As mentioned in Chapter 3, patch size is inversely proportional to frequency so the larger patch operates at the lower frequency. The bandwidth of resonance associated with the top patch is larger since this patch takes advantage of a thicker substrate, compared to the lower patch. 
5. Aperture shape size: The aperture length controls the coupling to the feedline and to the bottom patch. A longer aperture increases the coupling but also increases the back lobe radiation. Aperture width also controls the coupling, but not as much as the length. In order to achieve circular polarization and excite two orthogonal linear polarizations with a $90^{\circ}$ phase shift, two off-centered slots [25] or a cross slot should be used. For this design a cross slot was chosen [6].

6. Feed substrate thickness and permittivity: These factors control the coupling of the feedline to the aperture. For microstrip feeding these factors also control the guided wavelength and, therefore, the feedline dimensions.

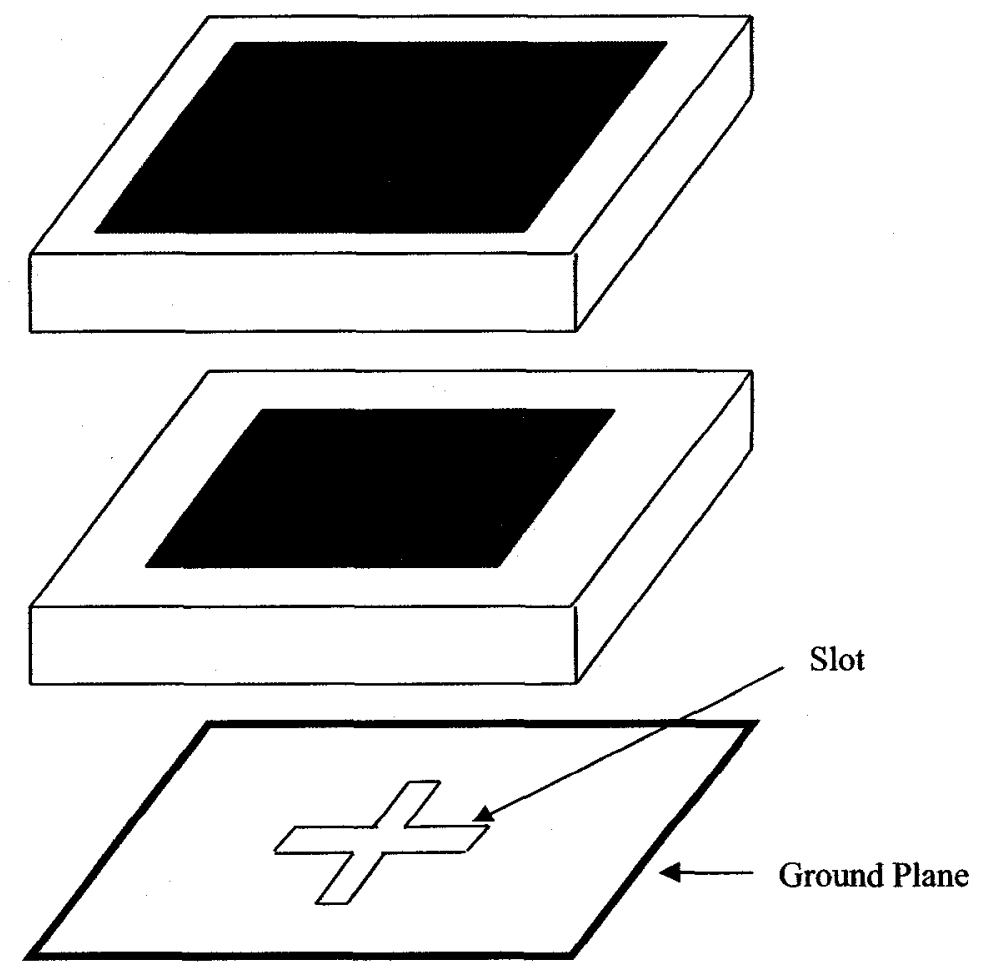

Figure 4.5: Selected geometry of the aperture-coupled stacked GPS antenna 


\subsubsection{Design Approach}

The design approach presented in this section is based on the results of a fullwave analysis presented by Targonski, Waterhouse, and Pozar [7] for a wide-band aperture-coupled stacked antenna. The configuration shown in Figure 4.1 consists of three resonators, two patches and the aperture. There is a mutual coupling between each resonator and by adjusting the resonator parameters, substrate thickness and permittivity the mutual coupling can be adjusted. Each of the three resonators has its own impedance loop and the goal is to optimize these loops so that the desired frequencies are close to the center of the Smith chart (VSWR <2). A typical Smith chart impedance locus of an aperture-coupled stacked patch antenna is shown in Figure 4.6 [7]:

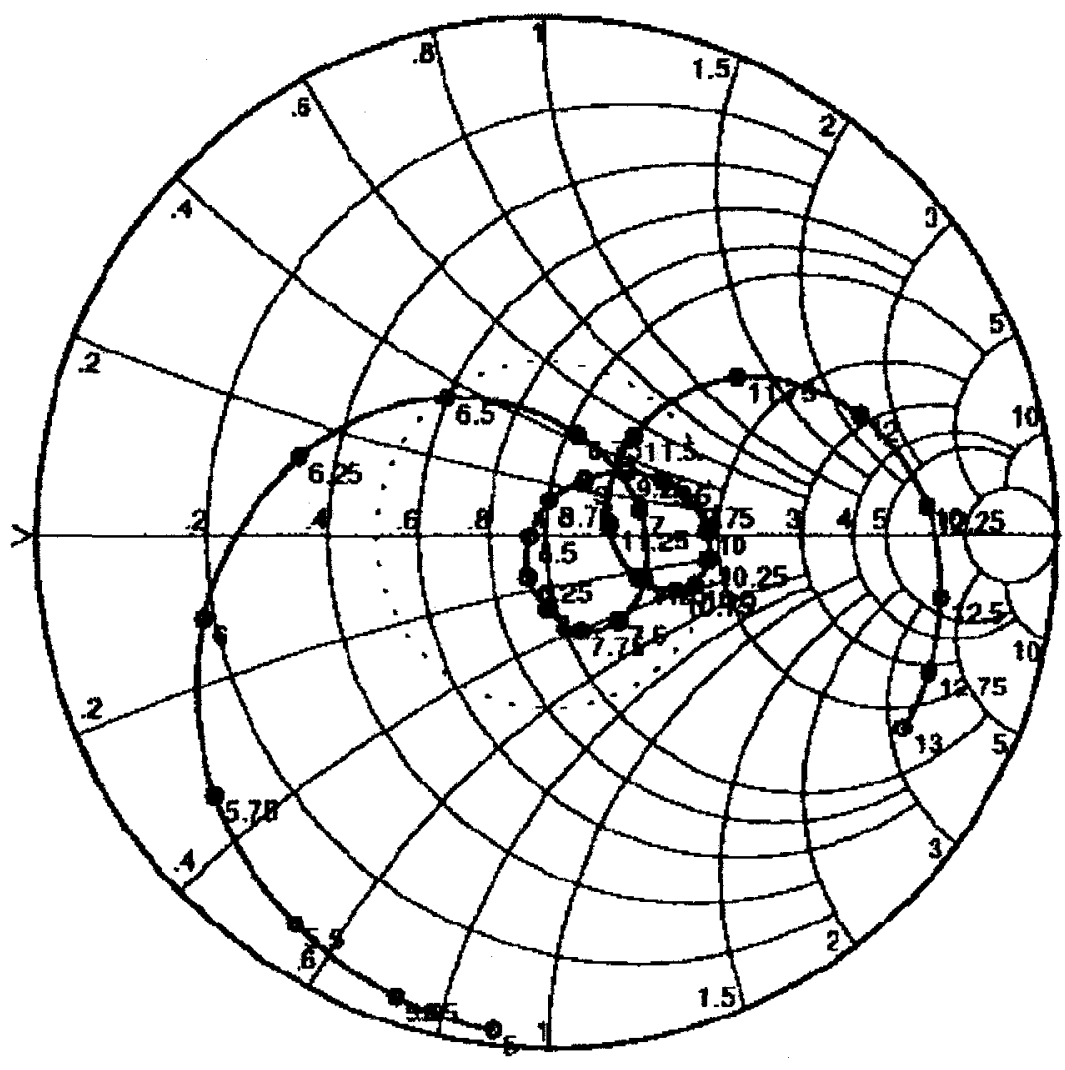

Figure 4.6: Typical impedance locus of an aperture-coupled stacked patch antenna [7] 
It is shown that this impedance locus has two closed narrow loops at the center of the Smith chart resulting in a wide bandwidth. In this discussion we consider that the lower patch is smaller than the upper patch; therefore the high frequency loop is due to the coupling of the lower patch and the slot, while the low frequency loop is due to the coupling between the upper and lower patch. These narrow loops are a result of weak coupling between the resonators and the wider loops are due to overcoupling. The design procedure for an aperture-coupled stacked patch antenna consists of changing parameters such as slot length, patch sizes, substrate materials, and thicknesses so that the impedance loops are close to each other and at the center of the Smith chart (VSWR <2).

At first we study the effect of slot length on the input impedance. It can be seen that slot length mostly affects the higher frequency loop, which represents the coupling between the lower patch and the aperture. Increasing the slot length increases the coupling producing a bigger high frequency loop. The lower patch size is an important design parameter since it contributes to coupling with both the aperture and the upper patch. Therefore both impedance loops are affected by changing the lower patch size. The upper patch size has similar affects, which shows that the relative patch sizes govern the impedance pattern rather than their individual size. The substrate thickness between the aperture and lower patch and also the lower patch and upper patch affects the mutual coupling between corresponding resonators. Thickening the substrate decreases the mutual coupling and could cause decoupling and also a very thin substrate produces overcoupling. It is important in the design to create a proper balance between the two mutual couplings and to avoid both undercoupling and overcoupling. 


\subsubsection{Feed Network Design}

The feed network must be designed so that right hand circular polarization (RHCP) and a matched input are obtained. The crossed slots are excited with equal amplitude and a $90^{\circ}$ phase difference. A parallel feeding configuration [6] was chosen for this purpose and is shown below:

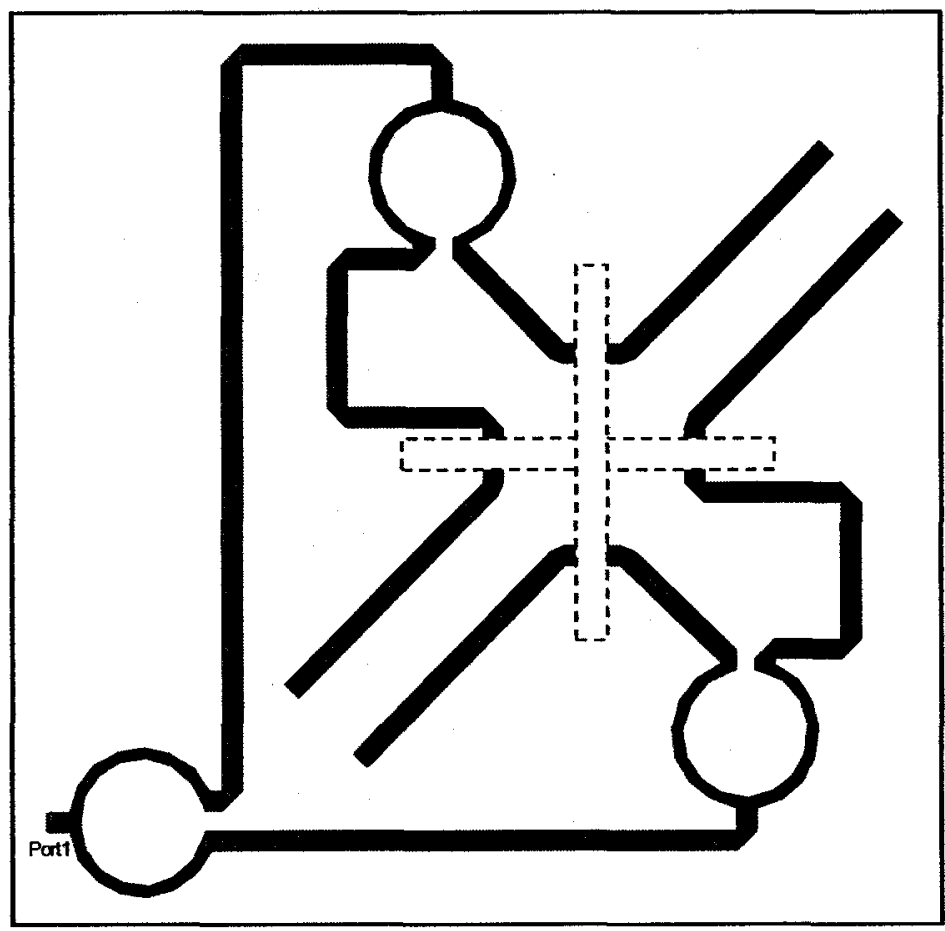

Figure 4.7: Microstrip feeding configuration for RHCP stacked-patch with cross aperture

This configuration provides accurate phase and amplitude for signal combining. As shown above each slot is fed by two feedlines at each end in opposite direction. There is a $180^{\circ}$ phase shift between lines at opposite ends of each arm. There is also a $90^{\circ}$ phase shift between each neighboring arm, which is produced by extending one arm of the 
output feedline by a quarter of a wavelength. This mechanism results in a sequential rotation in phase of $0^{\circ}, 90^{\circ}, 180^{\circ}$, and $270^{\circ}$ around the four feed points, producing circular polarization. Three Wilkinson power combiners are used to add the signals from the four feedlines. A Wilkinson combiner is a three-port device with the following scattering matrix [26]:

$$
[S]=\frac{-j}{\sqrt{2}}\left[\begin{array}{lll}
0 & 1 & 1 \\
1 & 0 & 0 \\
1 & 0 & 0
\end{array}\right]
$$

The two input ports are equally combined in amplitude and phase at the output port ( $3 \mathrm{~dB}$ up from each input). $Z_{0}$ is the nominal input $\&$ output impedance, in this case

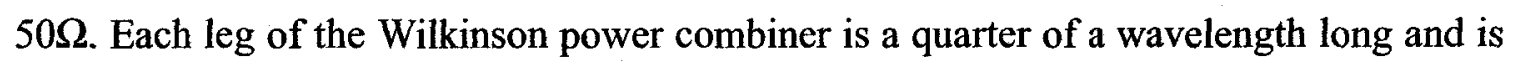
used to match $50 \Omega$ to $100 \Omega$; this quarter wave transformer has an impedance of $70.71 \Omega$, which is found by the following equation:

$$
Z=\sqrt{Z_{\text {Source }} Z_{\text {Load }}}=\sqrt{50 \times 100}=70.71 \Omega
$$

The $2 Z_{0}(100 \Omega)$ isolating chip resistor on the gap between the inputs is used to absorb reflected power caused by imbalances in the combiner. This resistor does not absorb any power if there is no imbalance. The schematic diagram of a Wilkinson combiner is shown in Figure 4.8 . 


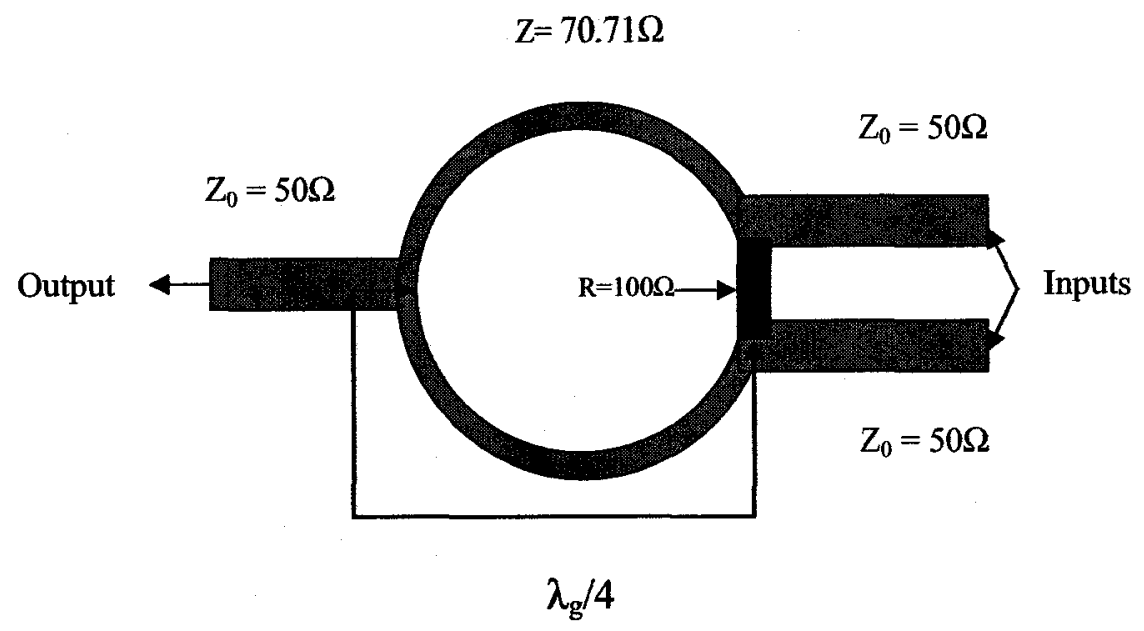

Figure 4.8: Wilkinson Power Combiner

The following figure shows the change in axial ratio with respect to the ratio of total power appearing at an input. It can be seen that axial ratio increases exponentially as power percentage decreases [27].

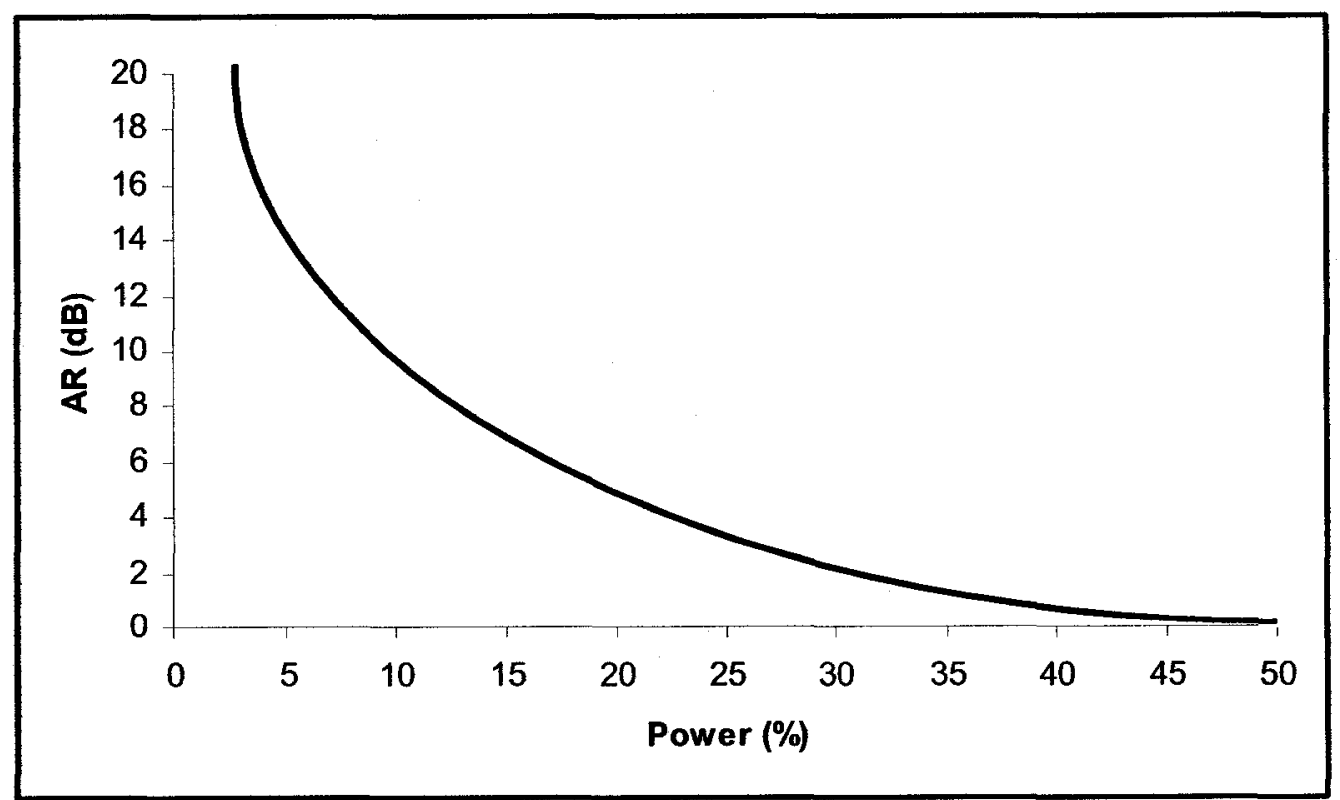

Figure 4.9: Axial Ratio vs. Power percentage at an input of a Wilkinson 
The two Wilkinson combiners closer to the aperture combine the power of two neighboring arms after introducing a $90^{\circ}$ phase shift, by having an input line a quarter of a wavelength longer than the other. The third combiner adds the outputs of the previous combiners after introducing a $180^{\circ}$ phase shift, by having an input line half a wavelength longer than the other. This configuration provides accurate phase and amplitude for signal combination but also requires enough space and precise design of Wilkinson combiners for acceptable circular polarization. The following figure shows the change in axial ratio with respect to the phase error. It can be seen that axial ratio increases exponentially as error increases [27].

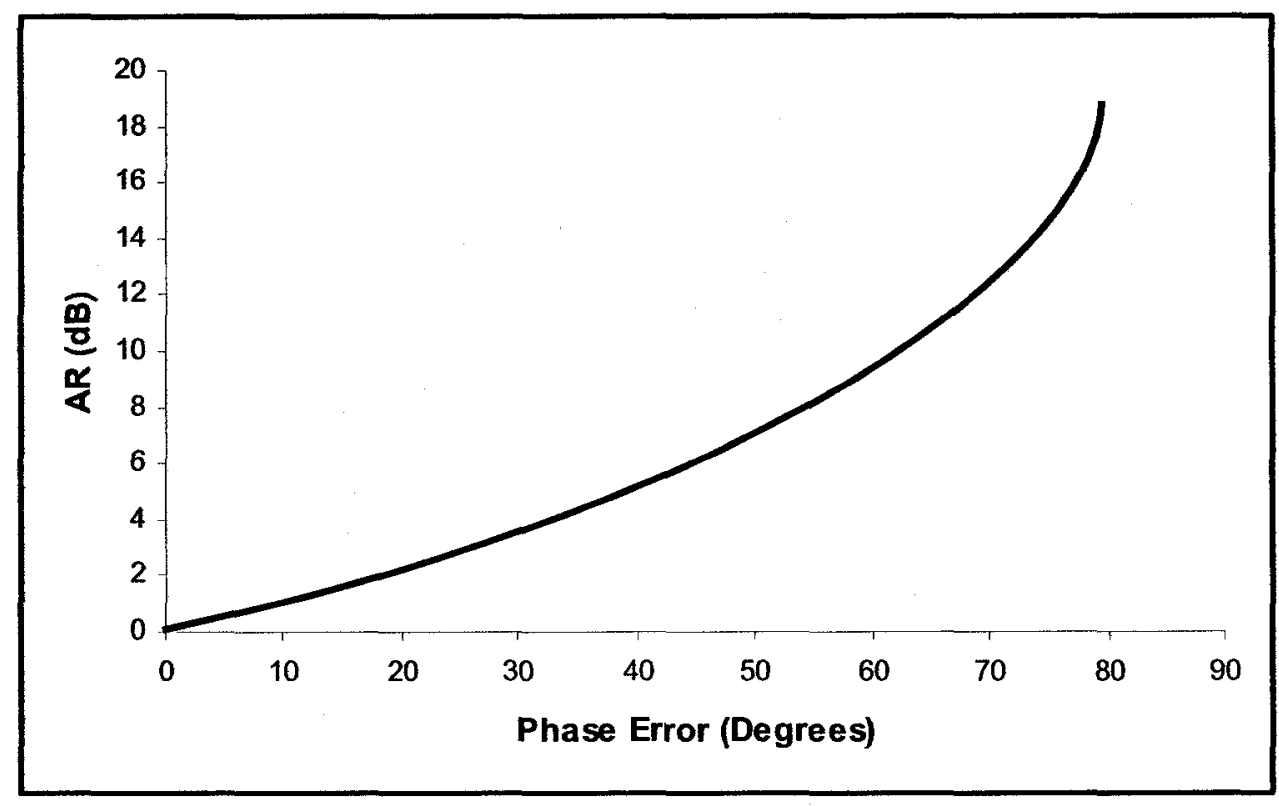

Figure 4.10: Axial Ratio vs. Phase Error 


\subsubsection{First Antenna Geometry:}

In order to determine the patch and substrate sizes it is best to start with a linearly polarized configuration with a single centered feedline. This configuration was shown in Figure 4.1. The dimensions determined from this starting point remain almost the same for the circular polarized design. The aperture size will require a slight increase after balanced feedlines are introduced.

The first antenna was designed so that the fabrication and material cost is minimal; therefore both patch substrates were made from a thick layer of Rohacell foam and a thin layer of FR4-epoxy for patch metallization. The feed substrate is also chosen to be a layer of FR4 with medium thickness. Rohacell is a Polymethacrylimide (PMI) hard foam, which is used as a core material for sandwich constructions. This material has permittivity close to air $(\sim 1)$. It also shows outstanding mechanical and thermal properties, it is inexpensive, light weight, and it has a high heat resistance. FR4, is an abbreviation for Flame Resistant 4, and type "4" indicates woven glass reinforced epoxy resin. This is a type of material mostly used for the making of printed circuit boards (PCBs). This material is inexpensive and has a permittivity of 4.7 at $1 \mathrm{MHz}$, and a loss tangent of 0.02 at $1 \mathrm{MHz}$. This combination of FR4 and Rohacell foam provides large bandwidth, a light weight structure, at minimum cost.

After a few empirical trials, the patch substrate thicknesses were fixed to $3.71 \mathrm{~mm}$, with $0.71 \mathrm{~mm}$ FR4 and $3 \mathrm{~mm}$ Rohacell foam. The feed substrate was set to $1.5 \mathrm{~mm}$ of FR4 material. Patch size determination was a tiresome process since the two patches interact 
with each other and with the aperture. The first set of measurements was done having one patch size fixed while varying the other. The main objective at this point of the design was to obtain a proper impedance match at the desired frequency bands. The two square patch dimensions were optimized and were set to be $73.2 \mathrm{~mm}$ and $89.6 \mathrm{~mm}$, keeping in mind that the larger patch was on top. The aperture length should provide a $50 \Omega$ input impedance and was found to be $4.9 \mathrm{~mm}$. The length of the tuning stub on the feedline was set to $41 \mathrm{~mm}$, which is a quarter of guided wavelength at the frequency of $1.4 \mathrm{GHz}$. The aperture length was increased to $52 \mathrm{~mm}$ with $6 \mathrm{~mm}$ width after the design was carried out towards circular polarization. These dimensions are summarized in the following table:

\begin{tabular}{|c|c|c|}
\hline Layer & Material & Dimension \\
\hline Top Patch & Copper & $89.6 \mathrm{~mm} \times 89.6 \mathrm{~mm}$ \\
\hline Top Substrate & FR4-epoxy/Rohacell & $0.71 \mathrm{~mm} / 3 \mathrm{~mm}$ \\
\hline Bottom Patch & Copper & $73.2 \mathrm{~mm} \times 73.2 \mathrm{~mm}$ \\
\hline Bottom Substrate & FR4-epoxy/Rohacell & $0.71 \mathrm{~mm} / 3 \mathrm{~mm}$ \\
\hline GroundPlane/ & Copper/ & $152.3 \mathrm{~mm} \times 152.3 \mathrm{~mm} /$ \\
Aperture & FR4-epoxy & $1.5 \mathrm{~mm}$ \\
\hline Feed Substrate & Copper & Parrallel Feed $/ 41 \mathrm{~mm}$ \\
\hline Feedline/Tuning Stub & & \\
\hline
\end{tabular}

Table 4.1: First Antenna Dimensions 
This antenna was simulated using Ansoft Designer 2.0, all the emperial tuning was done using this design tool. Ansoft Designer integrates simulation technologies of the Nexxim circuit simulator and of HFSS 3D electromagnetic field simulator. The following figures show a 2D and 3D view of this antenna in Ansoft Designer:
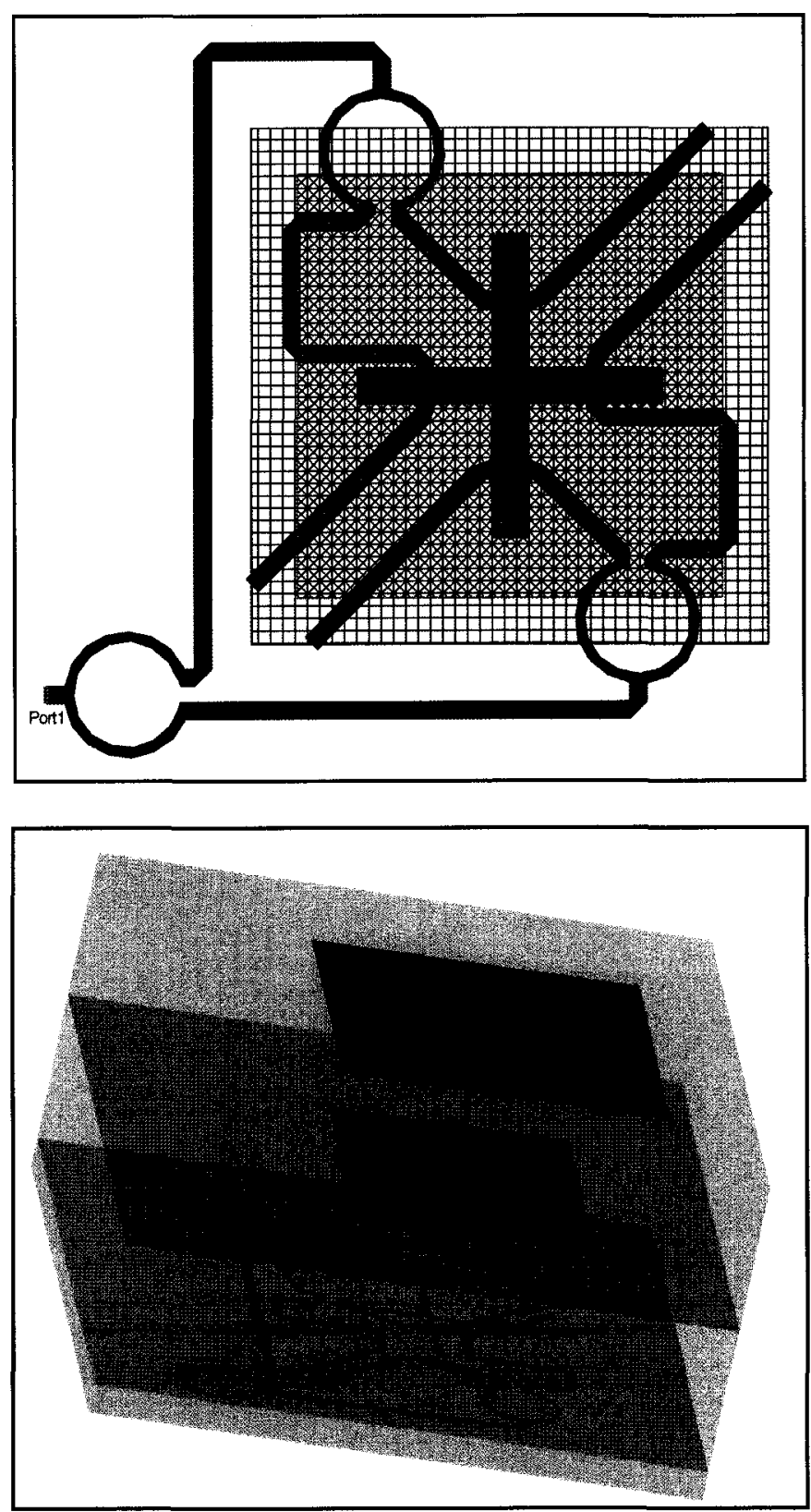

Figure 4.11: 2D and 3D view of the designed apreture-coupled stacked patch antenna in Ansoft Designer 
Figure 4.12 shows the simulated return loss of the antenna. It can be seen that a good impedance match at frequencies near L1, L2, and L5, and a mismatch at other neighboring frequencies is achieved. The lower frequency band associated with L2 and L5 has an impedance bandwidth of $120 \mathrm{MHz}(10 \%)$. The $\mathrm{Ll}$ frequency band has an impedance bandwidth of $35 \mathrm{MHz}(2.3 \%)$. The aperture is resonant at $1.5 \mathrm{GHz}$, which is not a suitable frequency for radiation. This frequency is also far enough from the two desired frequency bands.

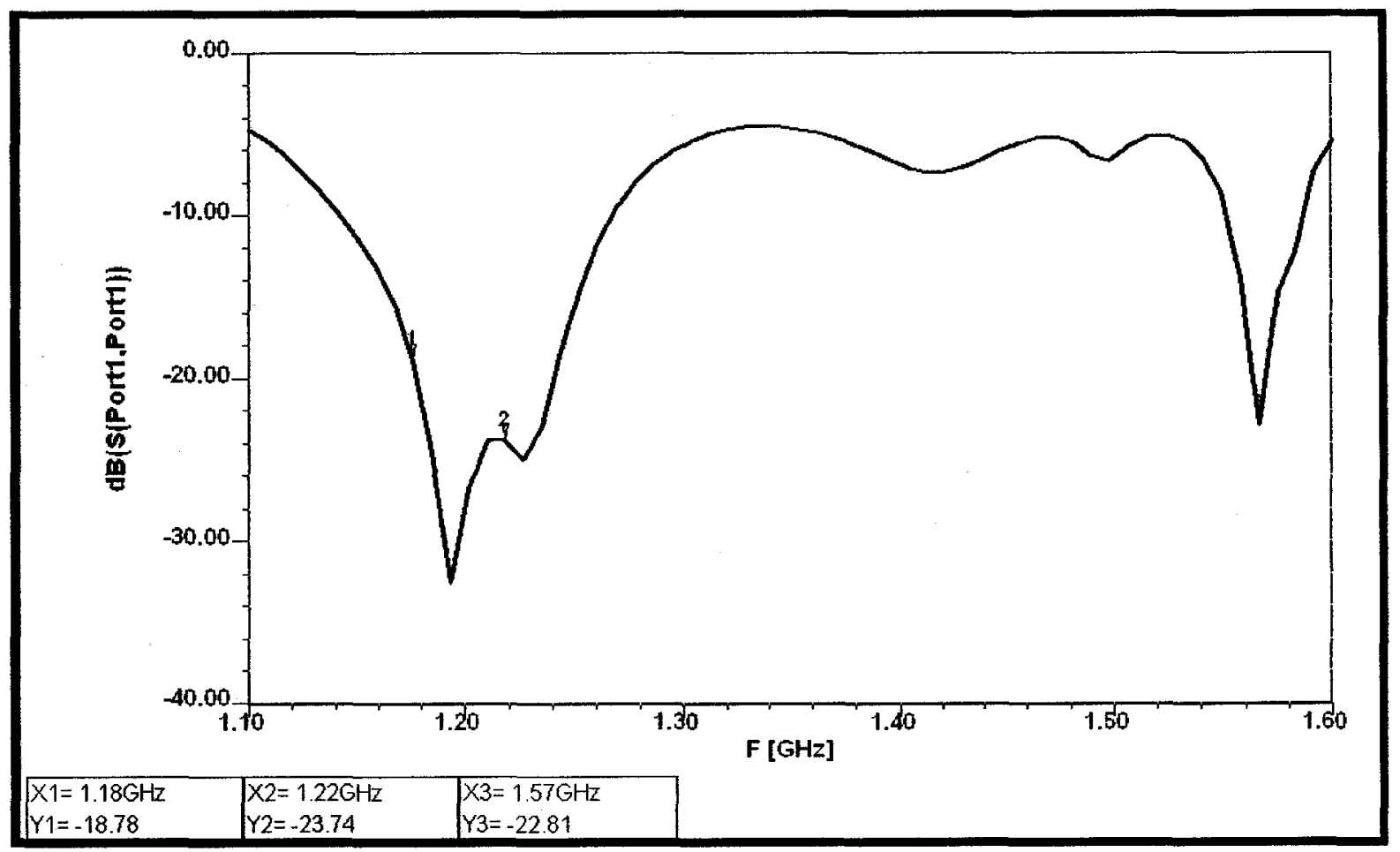

Figure 4.12: Simulated return loss of the first antenna measured at the input port. [Ansoft Designer] 
Figure 4.13 shows the impedance locus of the antenna. As described in section 4.4.2 the higher frequency loops is due to the coupling of the lower patch and the slot, while the lower frequency loop is due to the coupling between the upper and lower patch. Markers 1,2, and 3, show the location of the three desired frequencies. It can be seen that the higher frequency loop associated with $\mathrm{L} 1$ is larger and further from the center of the Smith chart, which is to be expected due to smaller bandwidth of this band. Also the lower frequency loop associated with L2 and L5 is smaller and more concentrated at the center of the Smith chart, producing a larger bandwidth.

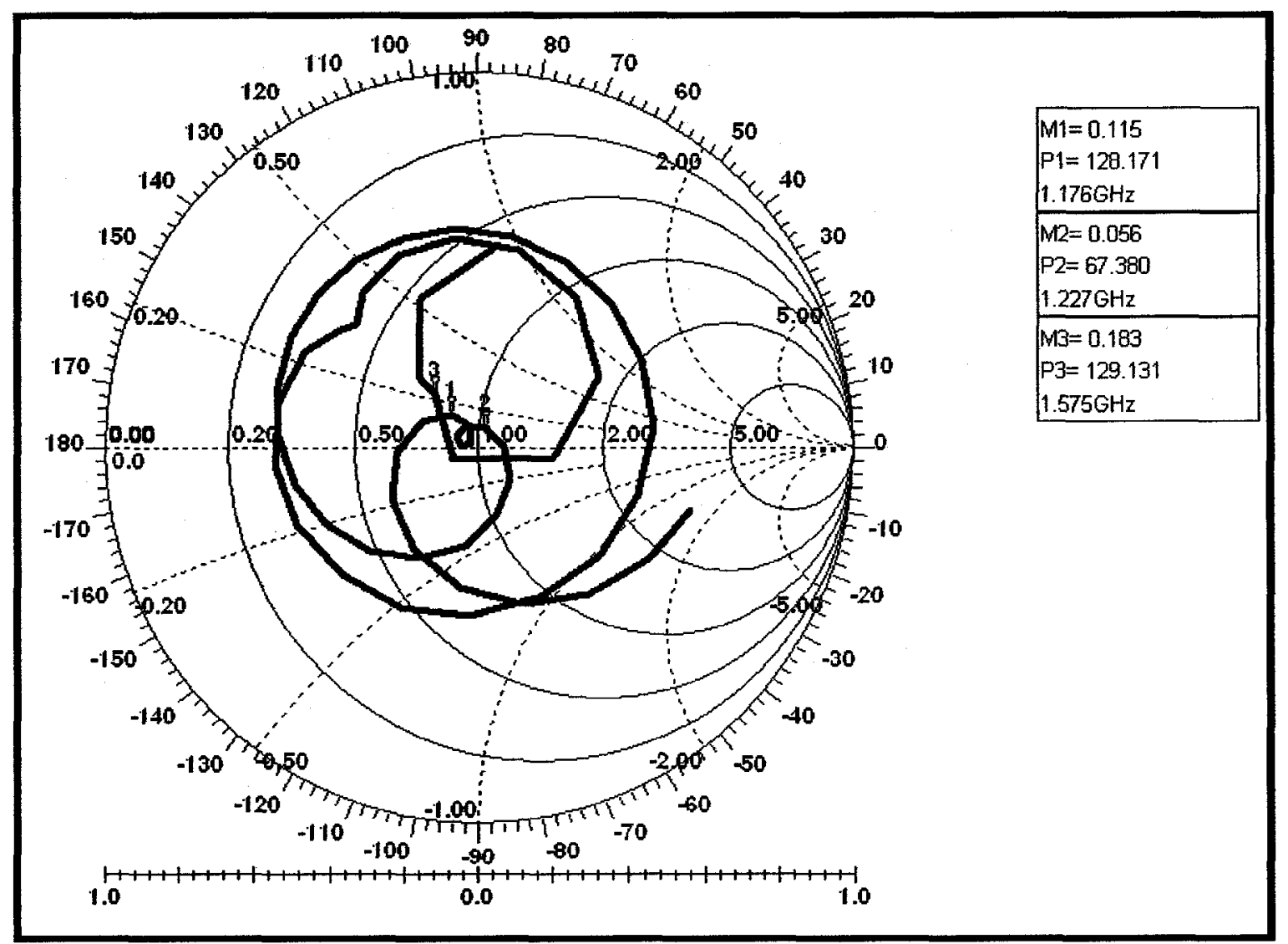

Figure 4.13: Simulated impedance locus of the first antenna [Ansoft Designer] 
Figure 4.14 shows the simulated gain of the antenna. It can be seen that a good impedance match at frequencies near L1, L2, and L5, and a mismatch at other neighboring frequencies result in a high gain for desired frequency bands. It can be seen that a gain of $5.33 \mathrm{~dB}$ at $\mathrm{L} 1,7.19 \mathrm{~dB}$ at $\mathrm{L} 2$, and $6.87 \mathrm{~dB}$ at $\mathrm{L} 5$ is achieved. These results confirm a proper patch radiation since the expected gain of a single patch antenna is about $5-7 \mathrm{~dB}$. Also, as expected, at the aperture resonance frequency of $1.5 \mathrm{GHz}$, the lowest gain is achieved.

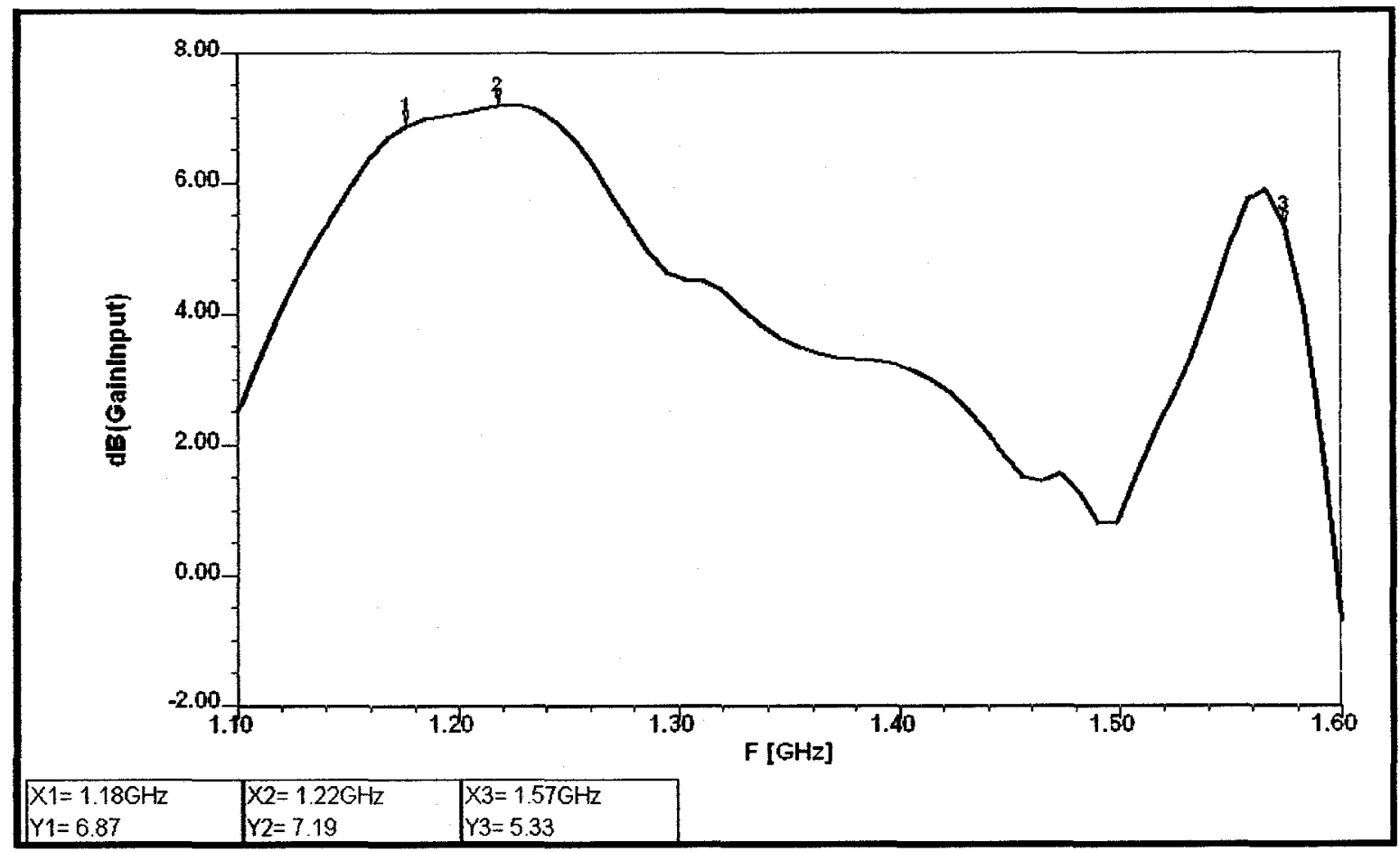

Figure 4.14: Simulated gain of the first antenna measured at the input port. [Ansoft Designer] 
Figure 4.15 shows the simulated axial ratio vs. frequency at broadside. It can be seen that an axial ratio of $1.05 \mathrm{~dB}$ at $\mathrm{L} 1,2.23 \mathrm{~dB}$ at $\mathrm{L} 2$, and $2.13 \mathrm{~dB}$ at $\mathrm{L} 5$ is achieved. These simulated axial ratios do not take into account the three $100 \Omega$ resistors, which will reduce these values significantly. Also it can be seen that at the aperture resonant frequency of $1.5 \mathrm{GHz}$, the highest axial ratio is achieved. Figure 4.16 (a) through (c) shows the axial ratio vs. different elevation angles (theta) in $\phi=0^{\circ}$ plane for the three desired frequencies. It can be seen that the axial ratio provides good CP over the beamwidth of $110^{\circ}$ for $\mathrm{L} 1,140^{\circ}$ for $\mathrm{L} 2$, and $150^{\circ}$ for $\mathrm{L} 5$.

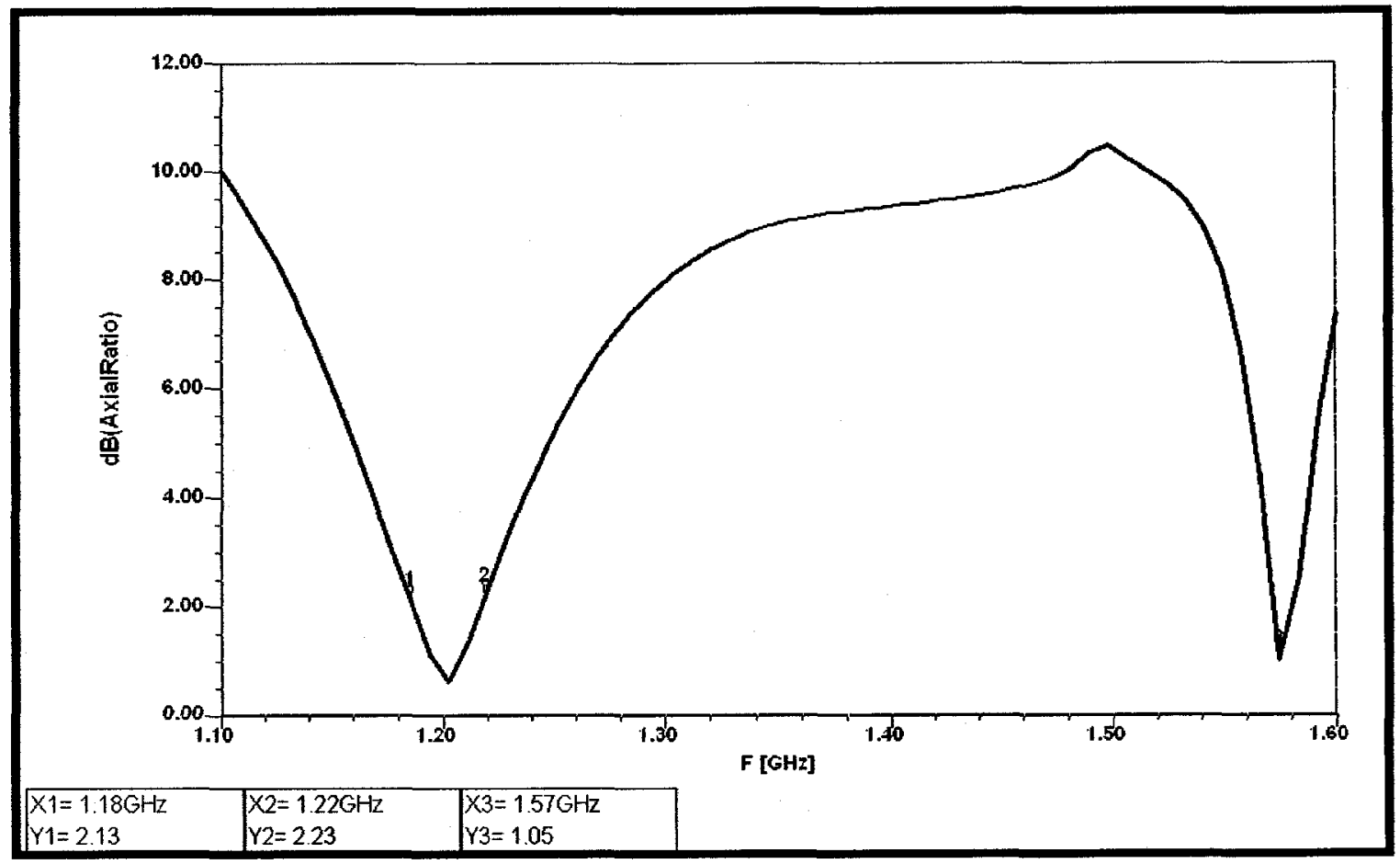

Figure 4.15: Simulated axial ratio vs. frequency of the first antenna At broadside. [Ansoft Designer] 


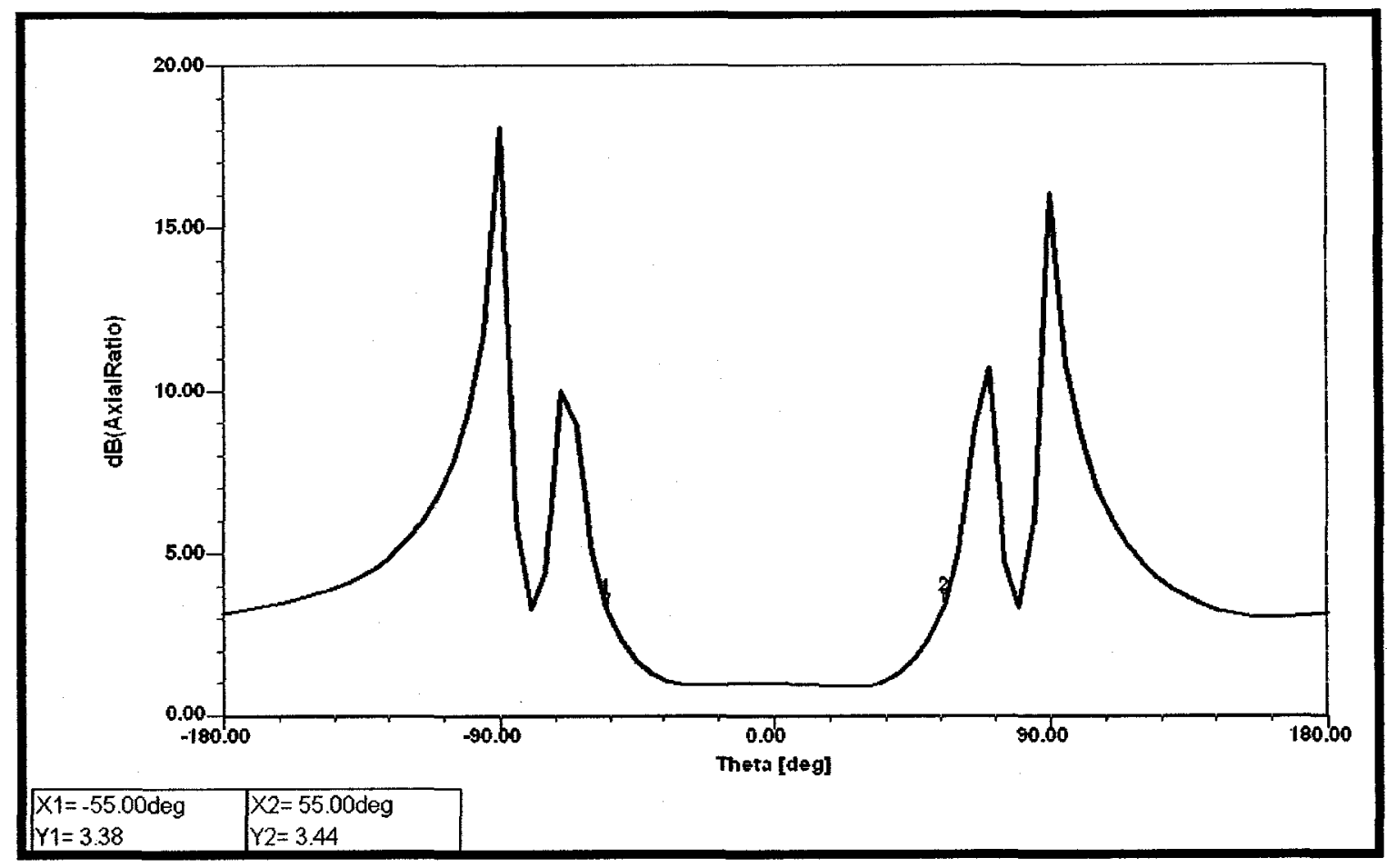

(a)

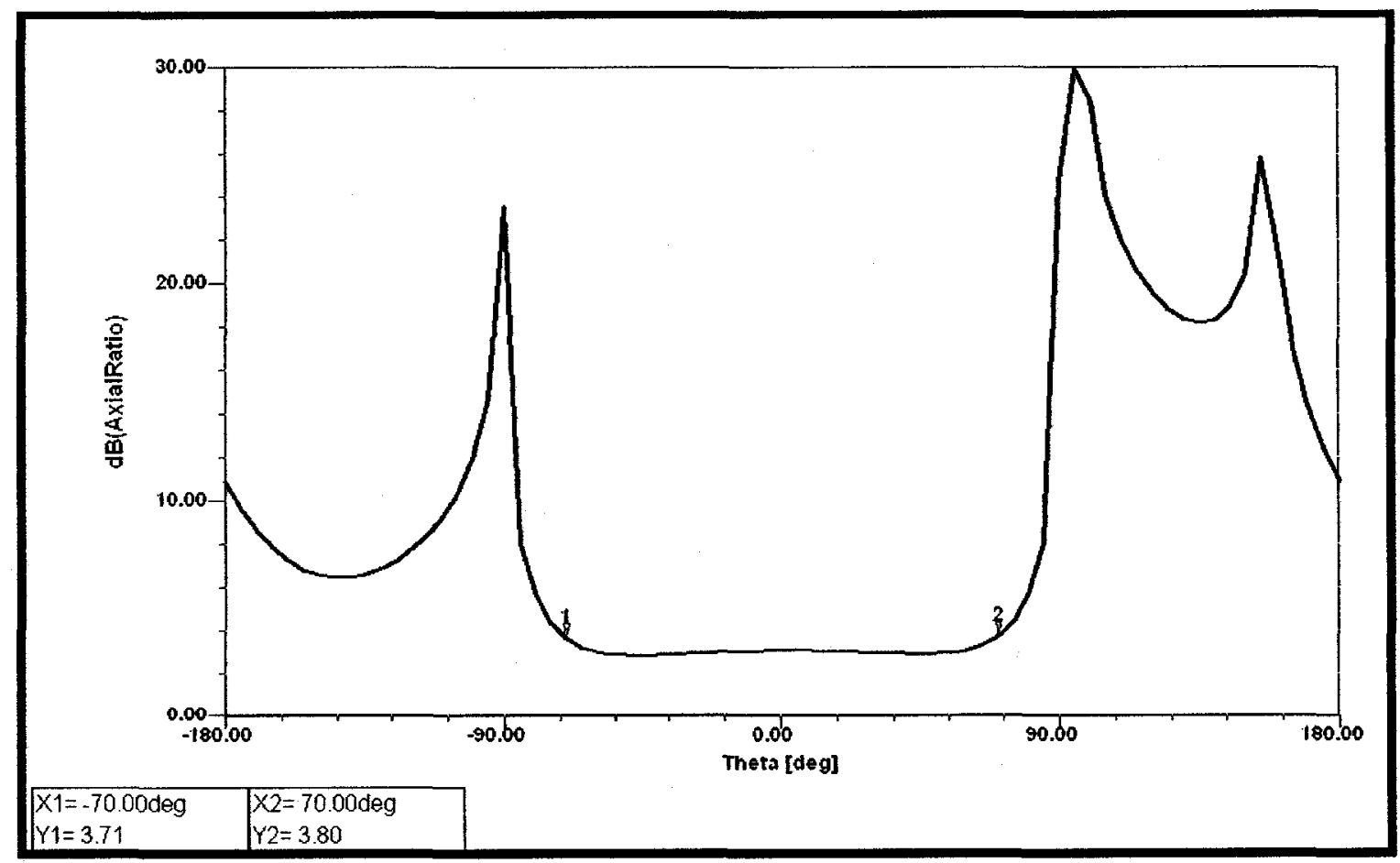

(b) 


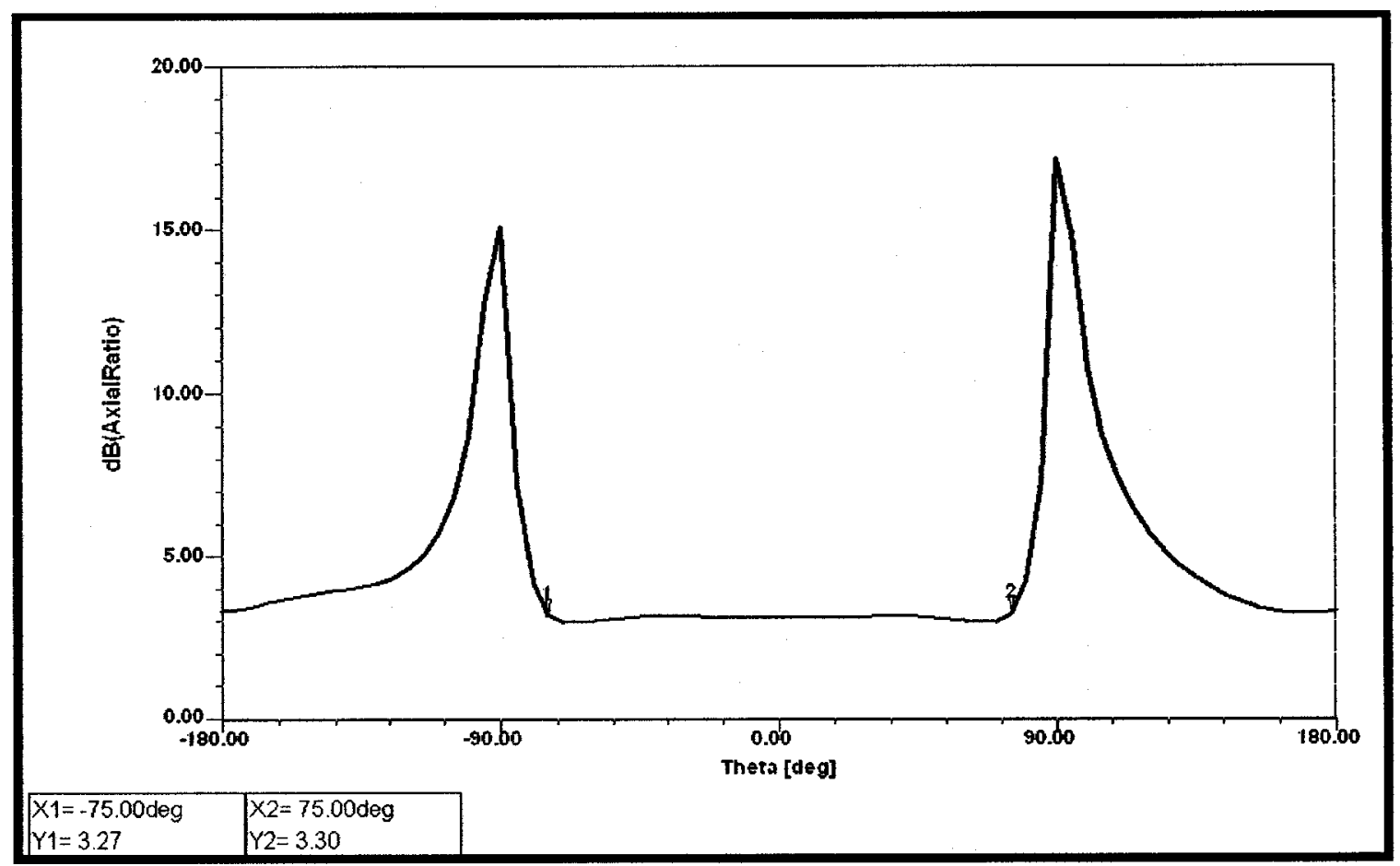

(c)

Figure 4.16: Simulated axial ratio vs. theta in $\phi=0^{\circ}$ plane.

(a) L1, (b) L2, (c) L5. [Ansoft Designer] 
Figure 4.17 (a) and (b) show the radiation patterns of the designed antenna at L1, in the $\phi=0^{\circ}$ and $\phi=90^{\circ}$ planes respectively. It can be seen that a gain of $5.33 \mathrm{~dB}$ at broadside is achieved. Other characteristics of these patterns are: a half power beamwidth (HPBW) of about $70^{\circ}$, null to null beamwidth (FNBW) of $180^{\circ}$, and a front to back ratio (FBR) of $8 \mathrm{~dB}$. Since this frequency band is to some extent close to the aperture resonance frequency of $1.5 \mathrm{GHz}$, it has suffered from back lobe radiation of maximum $-2 \mathrm{~dB}$. This back lobe radiation can be improved by shielding the back of the antenna by another ground plane. Figure 4.18 shows different views of the 3D radiation pattern at $\mathrm{L} 1$.

Figure 4.19 (a) and (b) show the radiation patterns at L2, in the $\phi=0^{\circ}$ and $\phi=$ $90^{\circ}$ planes respectively. It can be seen that a gain of $7.2 \mathrm{~dB}$ at broadside is achieved. Other characteristics of these patterns are: HPBW of about $80^{\circ}$, FNBW of $180^{\circ}$, and a FBR of 21dB. The FBR has improved significantly at this frequency.

Figure 4.20 (a) and (b) show the radiation patterns at L5, in the $\phi=0^{\circ}$ and $\phi=$ $90^{\circ}$ planes respectively. It can be seen that a gain of $6.87 \mathrm{~dB}$ at broadside is achieved. Other characteristics of these patterns are: HPBW of about $90^{\circ}$, FNBW of $180^{\circ}$, and a FBR of $18 \mathrm{~dB}$. Figure 4.21 shows different views of the $3 \mathrm{D}$ radiation pattern at $\mathrm{L} 2$, which is very similar to the ones for L5. These 3D patterns show that there is not a significant amount of energy concentrated in the back lobe. All the simulated radiation patterns provide sharp nulls at low elevation angles, which makes this antenna suitable for FRPA anti-jamming technique. The null positions are expected to shift from $\theta=90^{\circ}$, since the simulation method considers infinite substrate and ground plane. 


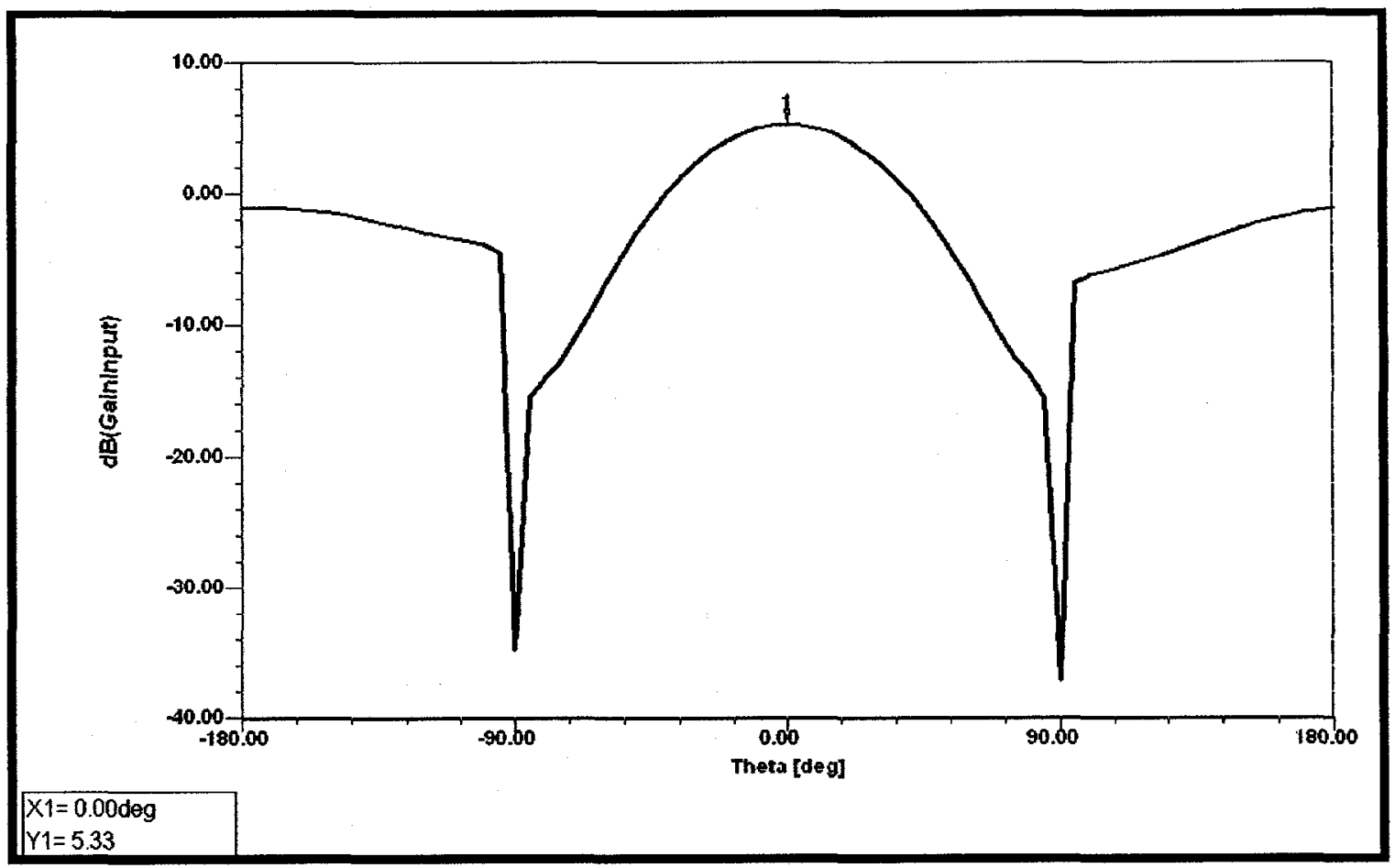

(a)

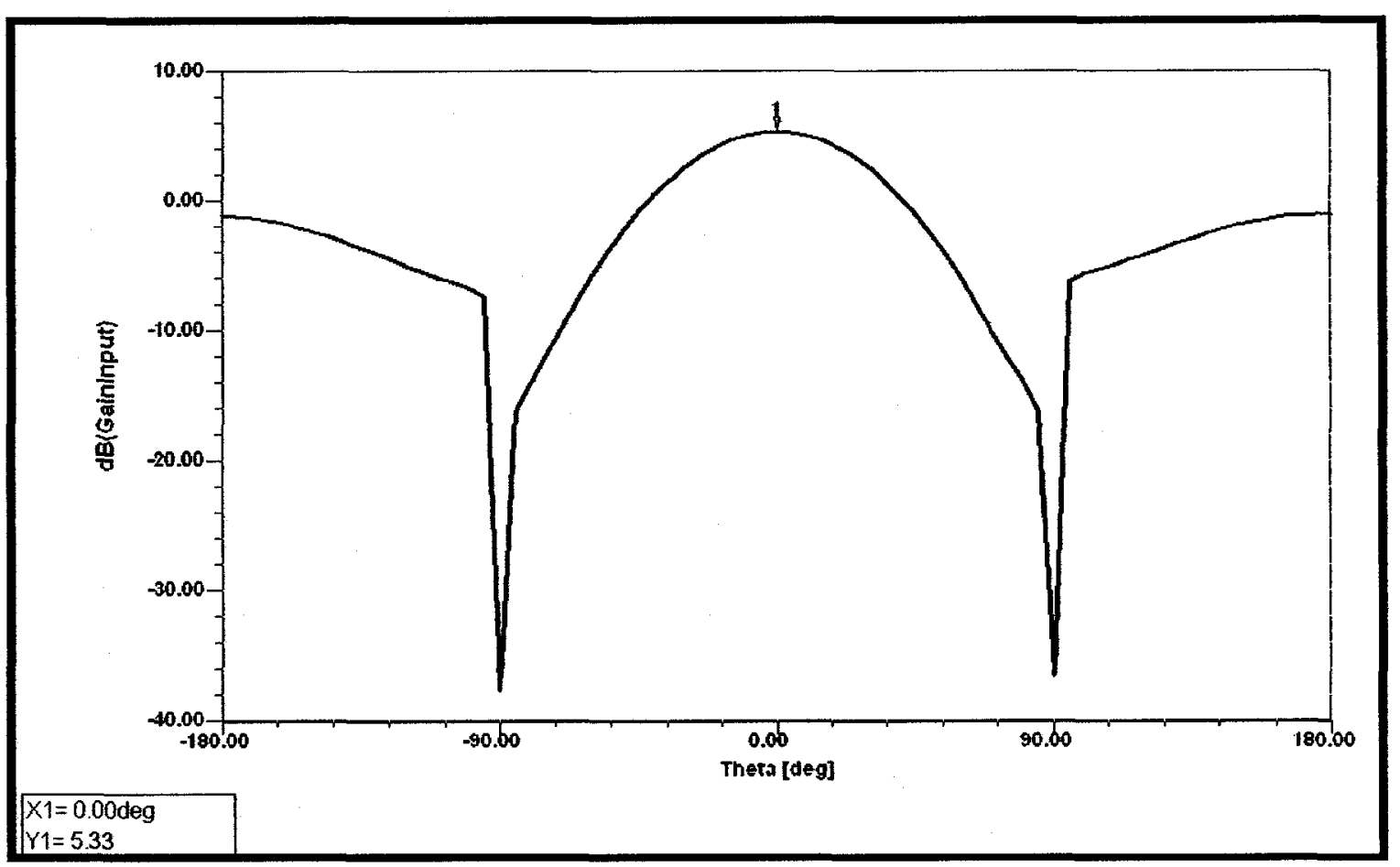

(b)

Figure 4.17: Simulated radiation pattern for $L 1$;

(a) $\phi=0^{\circ}$, (b) $\phi=90^{\circ}$. [Ansoft Designer] 

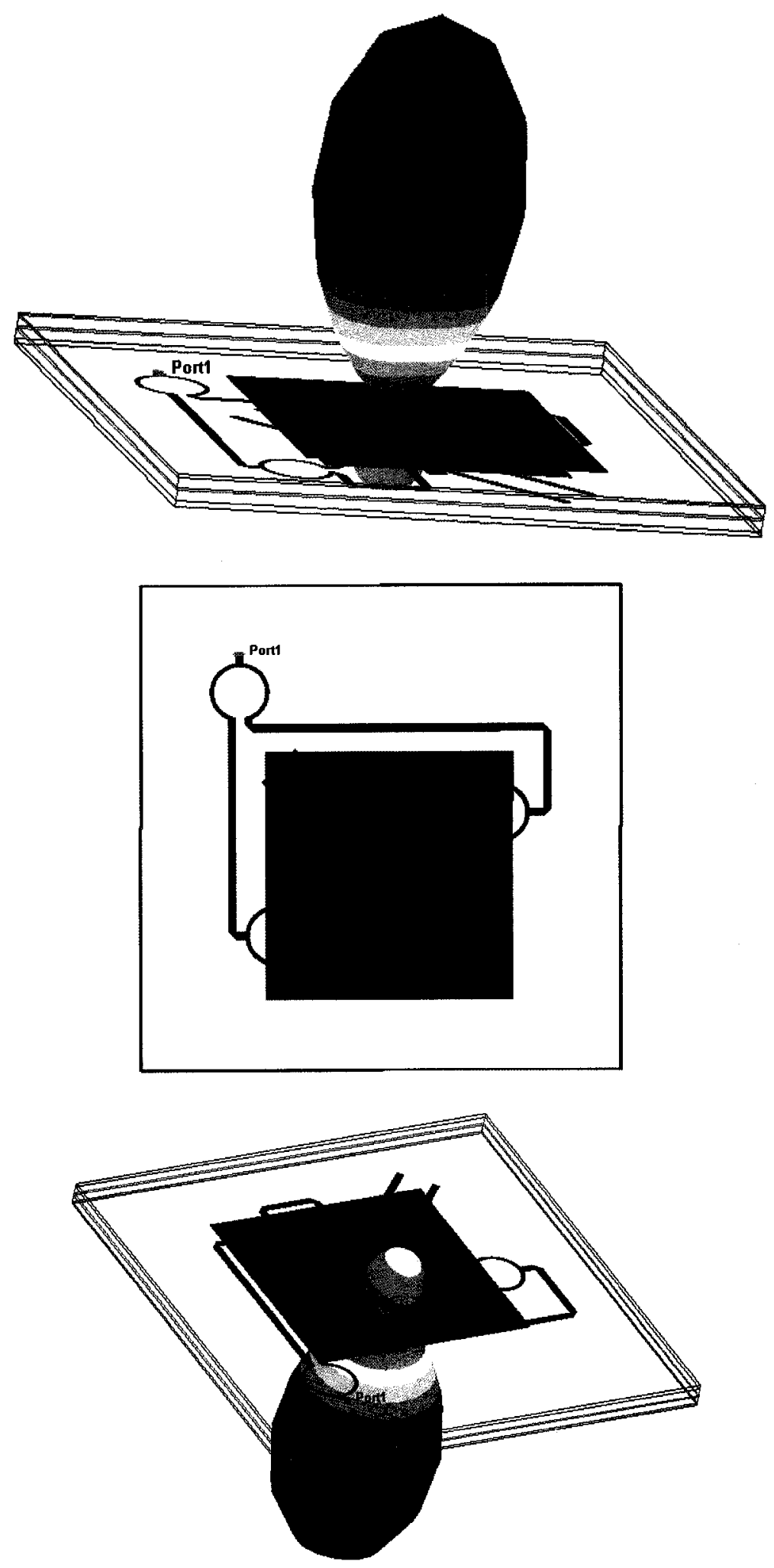

Figure 4.18: Side, Top, and Bottom, 3D radiation pattern at $\mathrm{L} 1$. [Ansoft designer] 


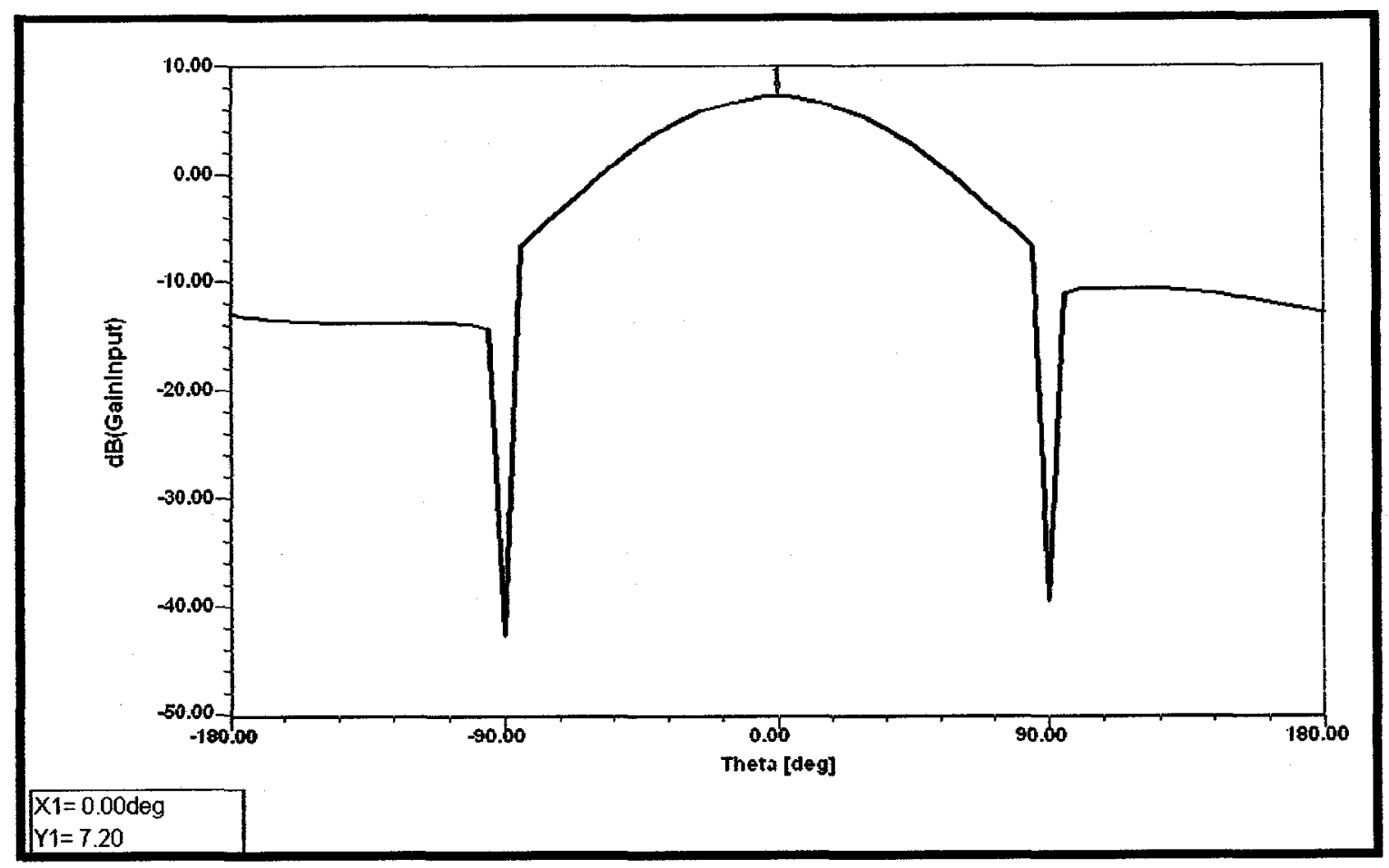

(a)

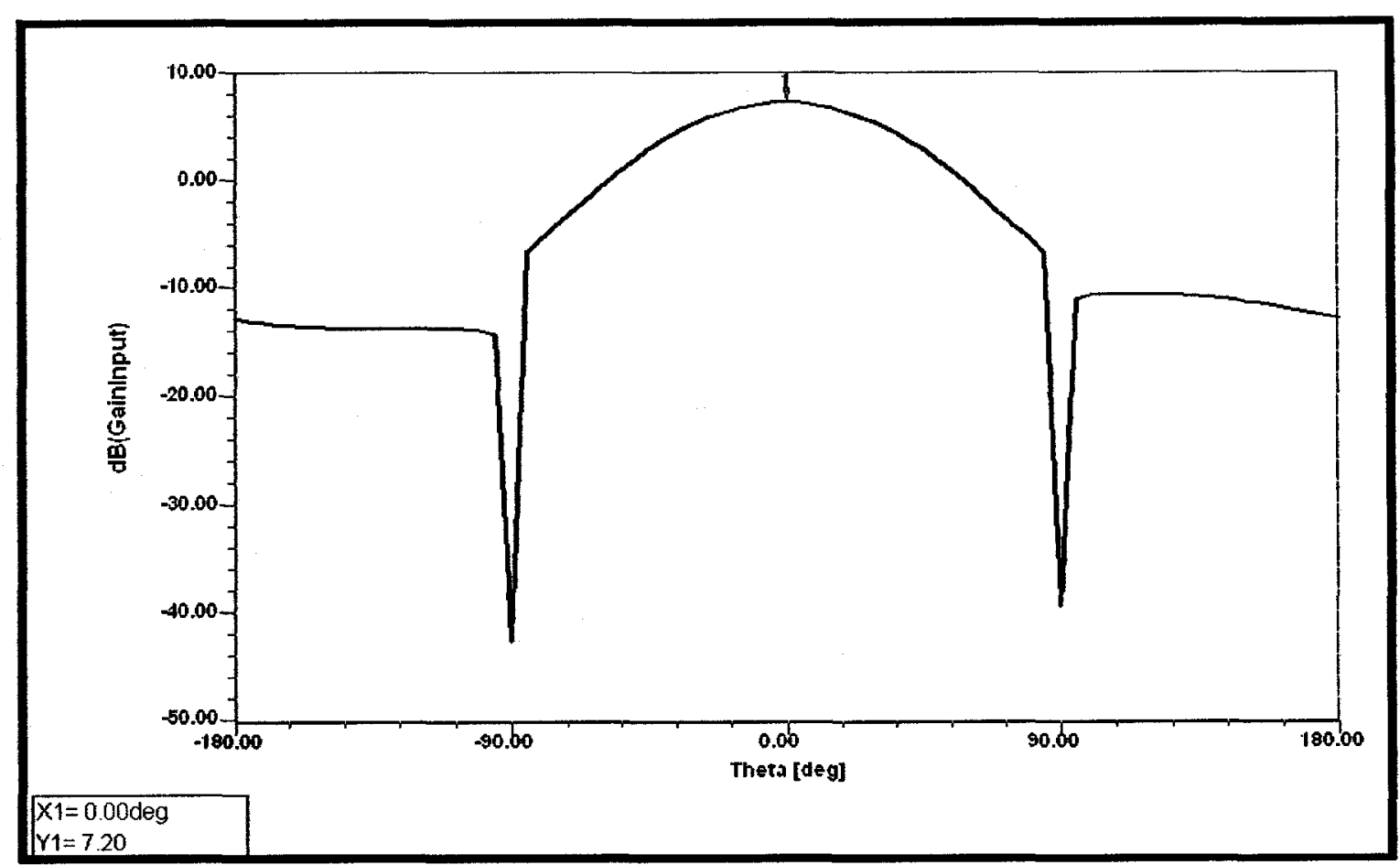

(b)

Figure 4.19: Simulated radiation pattern for $L 2$;

(a) $\phi=0^{\circ}$, (b) $\phi=90^{\circ}$. [Ansoft Designer] 


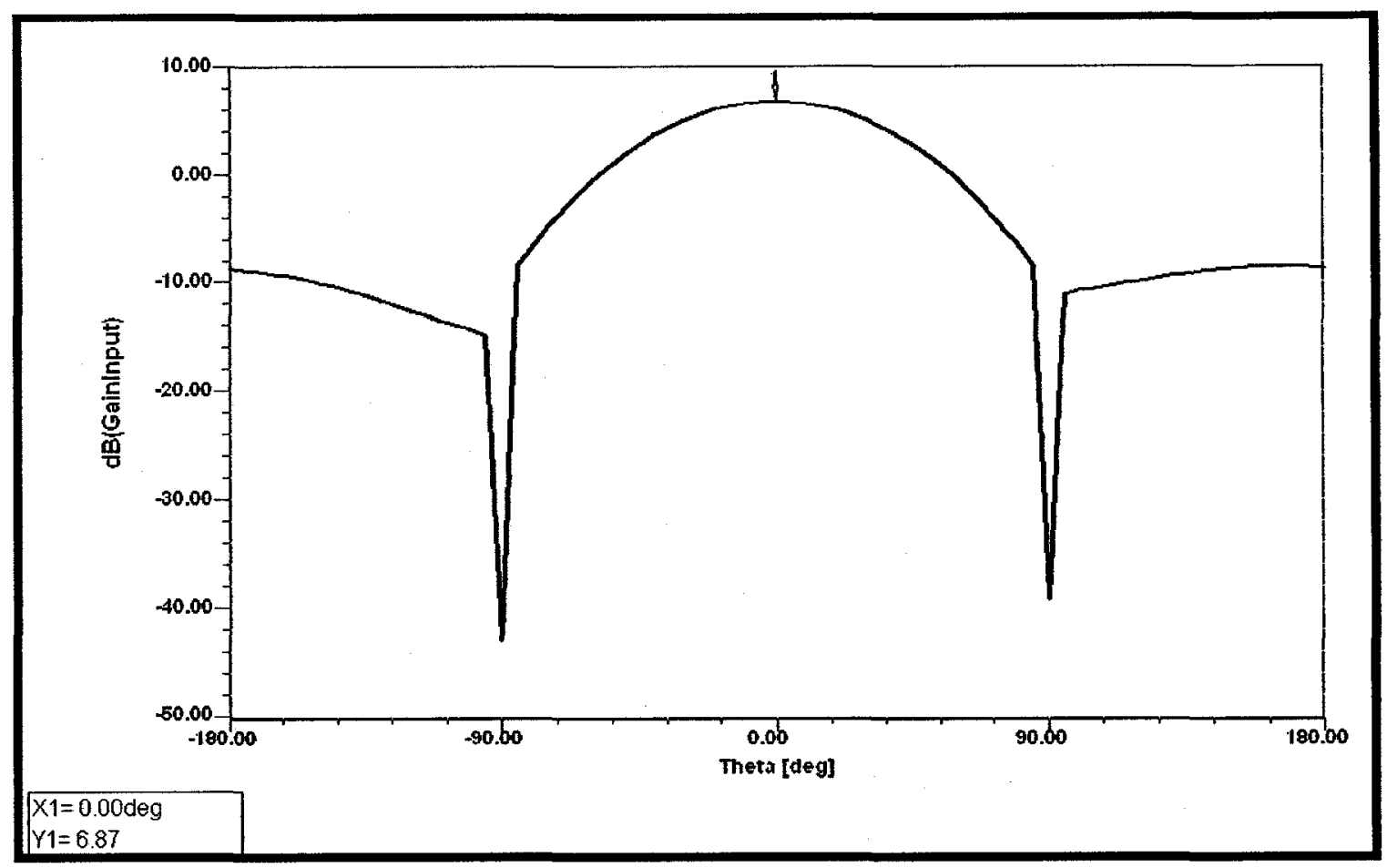

(a)

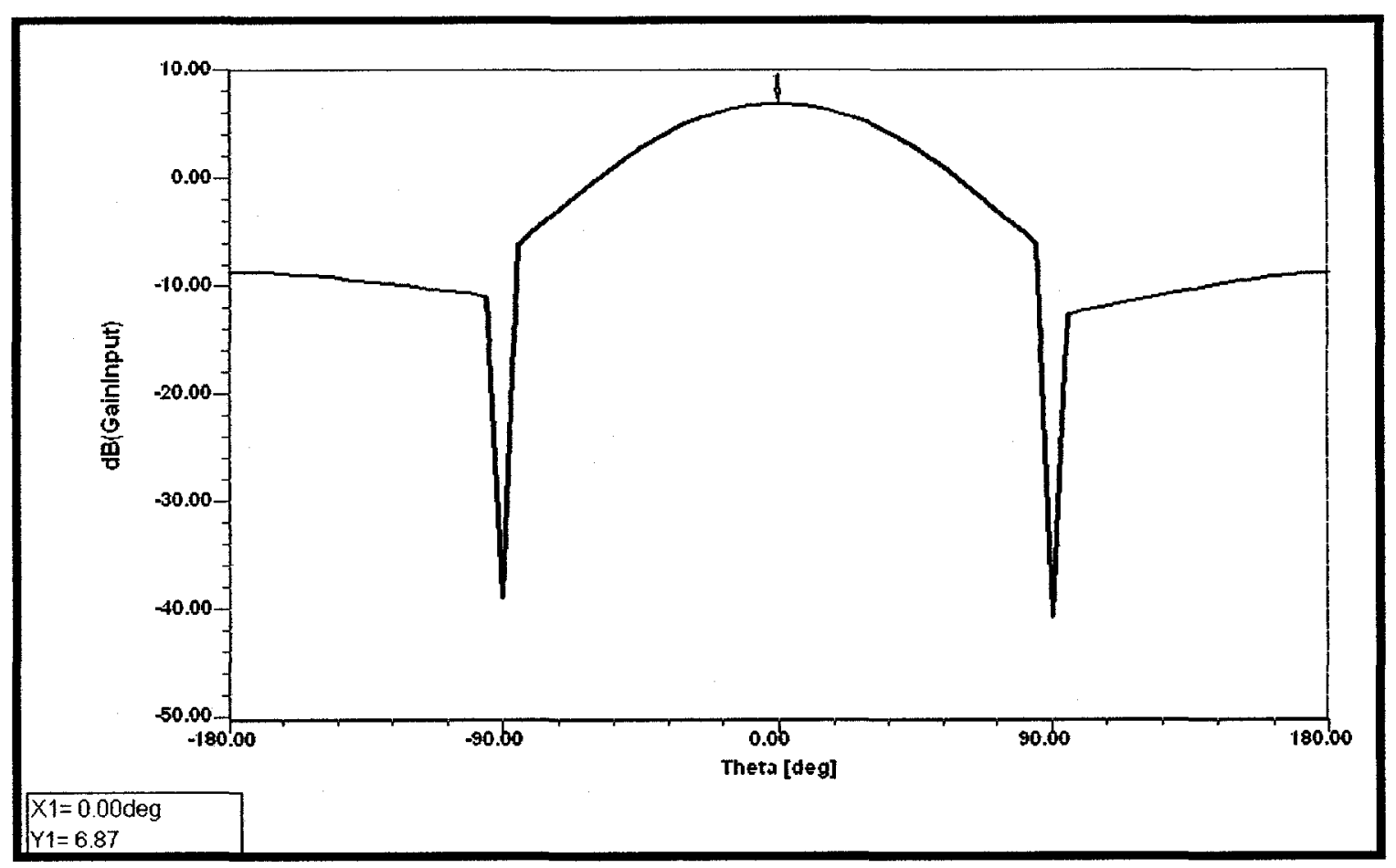

(b)

Figure 4.20: Simulated radiation pattern for $\mathrm{L} 5$;

(a) $\phi=0^{\circ}$, (b) $\phi=90^{\circ}$. [Ansoft Designer] 

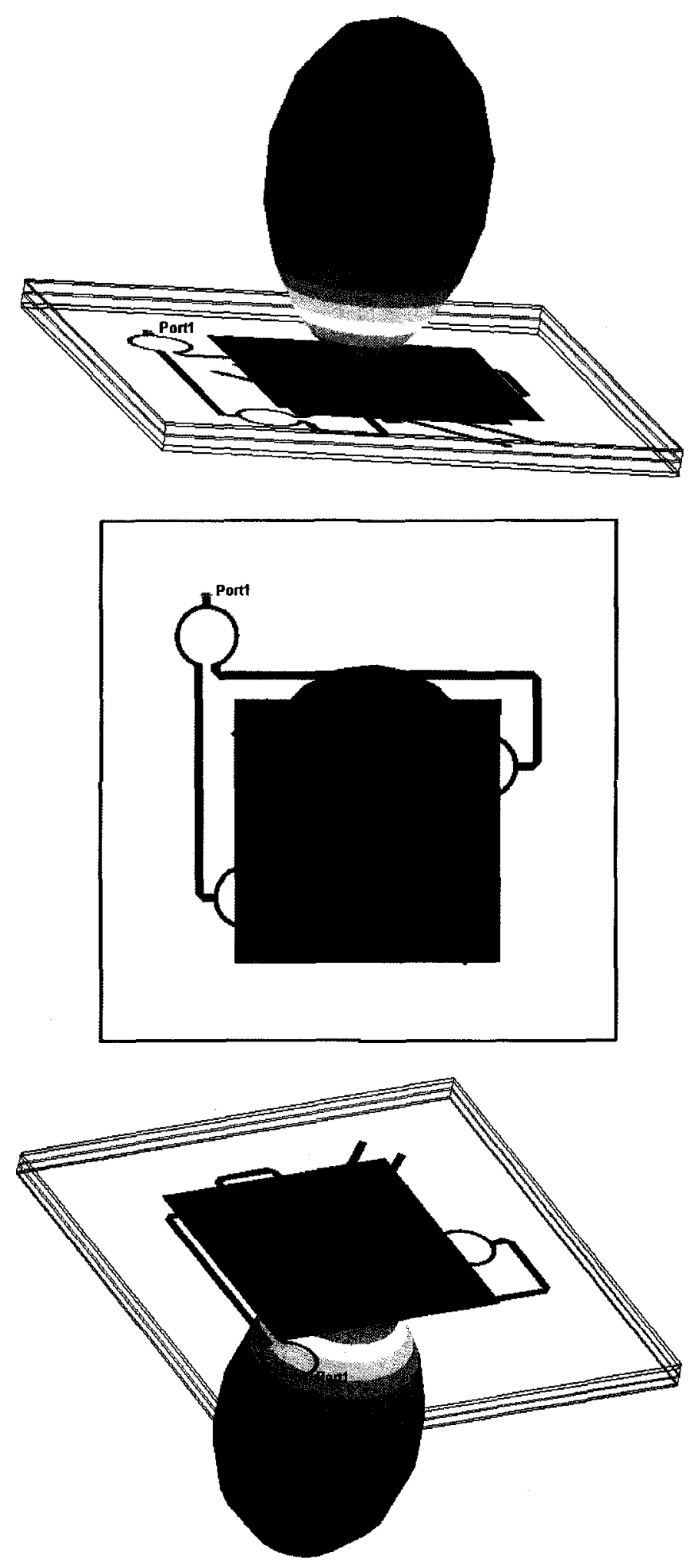

Figure 4.21: Side, Top, and Bottom, 3D radiation pattern

(Similar for both L2 and L5). [Ansoft designer] 


\subsubsection{Second Antenna Geometry:}

The reason for designing a second antenna was to decrease the dimensions of the first antenna. This size improvement requires substrate materials with higher permittivity since the microstrip line dimensions decrease as permittivity increases. This change in material results in a heavier, more expensive antenna, but also makes the antenna more attractive and suitable in an array configuration. Similar actions were taken to design this antenna, starting with a linear polarized design.

In order to minimize the size of the first antenna, both the patches and the feedline must shrink in size. To shrink the patch sizes Arlon CLTE was chosen as the patch substrate. CLTE is an abbreviation for Controlled Low Thermal Expansion. This material is a ceramic-filled, woven fiberglass reinforced PolyTetraFluoroEthylene (PTFE) composite; engineered to produce a dimensionally and electrically stable laminate material with a nominal dielectric constant of 2.96 and a loss tangent of 0.0023 at $10 \mathrm{GHz}$. Arlon CLTE has a very high temperature stability, which optimizes circuit performance in many microwave applications, such as phased array antennas.

Reducing the feed network is more critical and requires a substrate material with a very high permittivity. For this purpose Rogers TMM10i (Thermoset ceramic loaded plastic) was chosen. This substrate is a ceramic thermoset composite designed for high quality microstrip line fabrication with a nominal dielectric constant of 9.8 and a loss tangent of 0.002 at $10 \mathrm{GHz}$. This material's polymer thermal properties were well matched so as to have stable electrical and mechanical performance. 
The patch substrate thicknesses were fixed to $3.175 \mathrm{~mm}$ of CLTE, thick enough to provide enough bandwidth for desired frequency bands. The feed substrate was set to $1.9 \mathrm{~mm}$ of RogersTMM10i. The patch sizes were determined following the same method as for the first antenna, keeping in mind that the main objective was to obtain a proper impedance match at the desired frequency bands. The two square patch dimensions were optimized and were set to be $46.6 \mathrm{~mm}$ and $63.6 \mathrm{~mm}$, with the larger patch on top. The aperture length required to provide a $50 \Omega$ input impedance and was found to be $3.5 \mathrm{~mm}$ in length and $1 \mathrm{~mm}$ in width. The length of the tuning stub on the feedline was set to $26.5 \mathrm{~mm}$, which was a quarter of guided wavelength at the mid-frequency of $1.4 \mathrm{GHz}$. The aperture length was increased to $3.8 \mathrm{~mm}$ after the design was carried out toward circular polarization. This antenna design resulted in a $30 \%$ size reduction compared to the first antenna. These dimensions make this antenna suitable for linear or planar array configuration. These dimensions are summerized in the following table:

\begin{tabular}{|c|c|c|}
\hline Layer & Material & Dimension \\
\hline Top Patch & Copper & $63.6 \mathrm{~mm} \times 63.6 \mathrm{~mm}$ \\
\hline Top Substrate & Arlon CLTE & $3.175 \mathrm{~mm}$ \\
\hline Bottom Patch & Copper & $46.6 \mathrm{~mm} \times 46.6 \mathrm{~mm}$ \\
\hline Bottom Substrate & Arlon CLTE & $3.175 \mathrm{~mm}$ \\
\hline $\begin{array}{c}\text { GroundPlane/ } \\
\text { Aperture }\end{array}$ & Copper/ & $105.5 \mathrm{~mm} \times 105.5 \mathrm{~mm} /$ \\
Feed Substrate & RogersTMM10i & $1.9 \mathrm{~mm}$ \\
\hline Feedline/Tuning Stub & Copper & Parrallel Feed $/ 26.5 \mathrm{~mm}$ \\
\hline
\end{tabular}

Table 4.2: Second Antenna Dimensions 
Figure 4.22 shows the simulated return loss of the second antenna. It can be seen that a good impedance match at frequencies near L1, L2, and L5, and a mismatch at other neighboring frequencies is achieved. The lower frequency band associated with L2 and L5 has an impedance bandwidth of $70 \mathrm{MHz}(6 \%)$. The $\mathrm{L} 1$ frequency band has an impedance bandwidth of $20 \mathrm{MHz}(2 \%)$. The aperture is resonant at $1.53 \mathrm{GHz}$, which is not a suitable frequency for radiation. This frequency also is far enough from the two desired frequency bands.

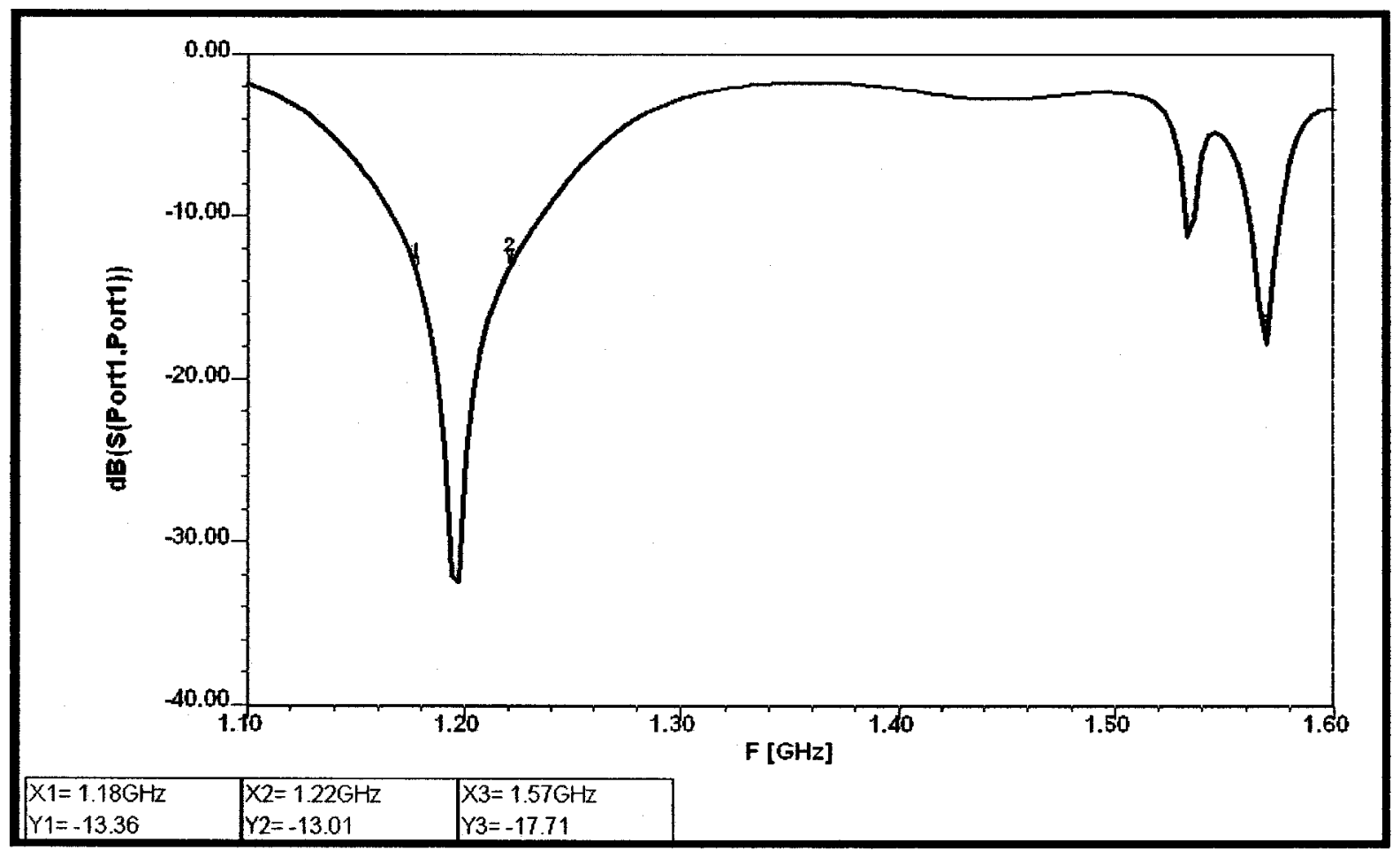

Figure 4.22: Simulated Return Loss of the second antenna measured at the input port. [Ansoft Designer] 
Figure 4.23 shows the impedance locus of the second antenna. The higher frequency loops is due to the coupling of the lower patch and the slot, while the lower frequency loop is due to the coupling between the upper and lower patch. Markers 1, 2, and 3, show the location of the three desired frequencies. It can be seen that the higher frequency loop associated with $\mathrm{Ll}$ is larger and further from the center of the Smith chart, which is to be expected due to narrower bandwidth of this band. Also the lower frequency loop associated with L2 and L5 is smaller and more concentrated at the center of the Smith chart, producing a larger bandwidth.

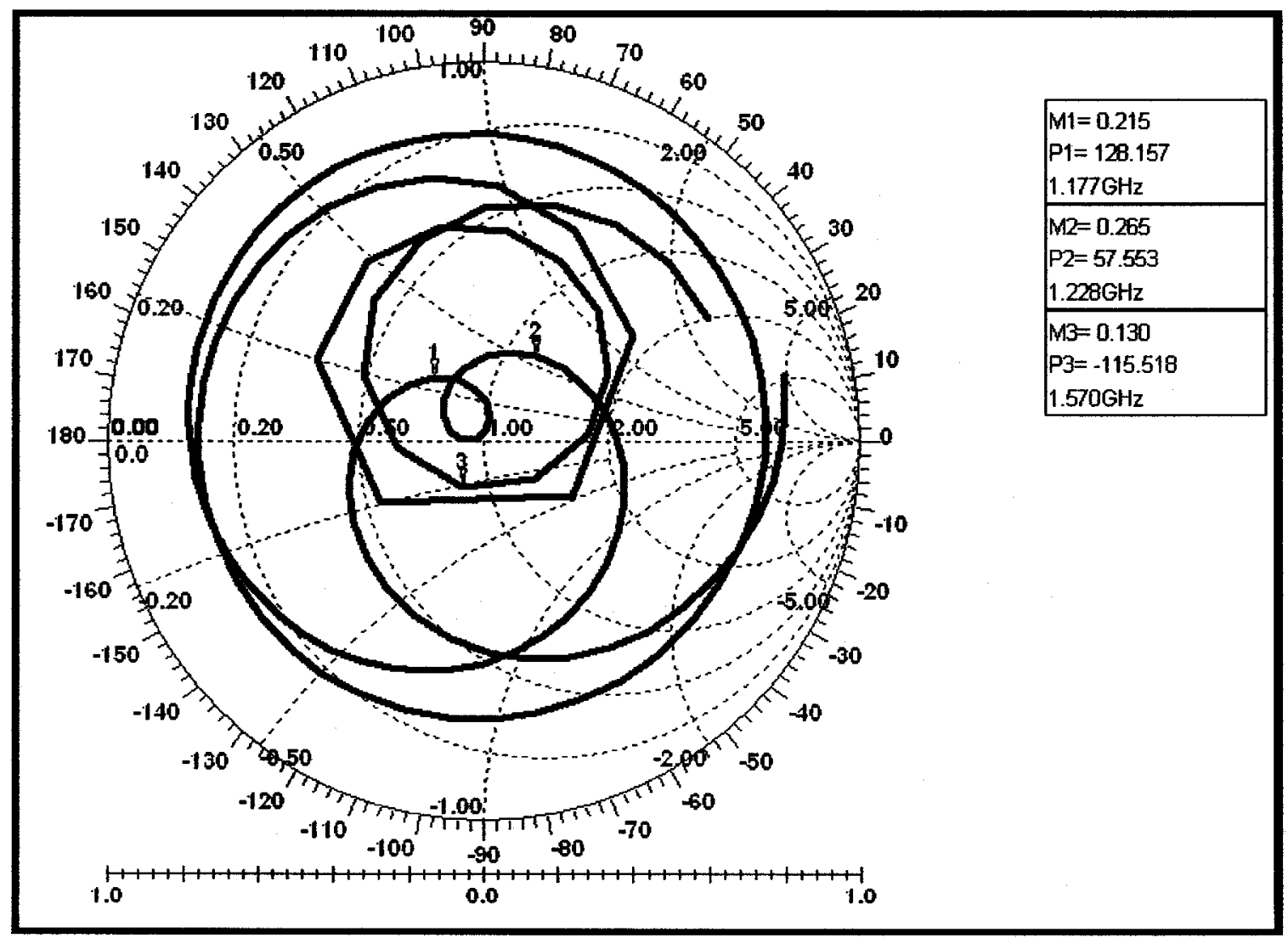

Figure 4.23: Simulated impedance locus of the second antenna [Ansoft Designer] 
Figure 4.24 shows the simulated gain of the second designed antenna. It can be seen that a good impedance match at frequencies near L1, L2, and L5, and mismatch at other neighboring frequencies results in a high gain for desired frequency bands. It can be seen that a gain of $5.33 \mathrm{~dB}$ at $\mathrm{L} 1,5.68 \mathrm{~dB}$ at $\mathrm{L} 2$, and $5.48 \mathrm{~dB}$ at $\mathrm{L} 5$ is achieved. These results confirm a successful design since the expected gain of a single patch antenna is about $5-7 \mathrm{~dB}$. Also as expected, at the aperture resonance frequency of $1.53 \mathrm{GHz}$ the lowest gain is achieved.

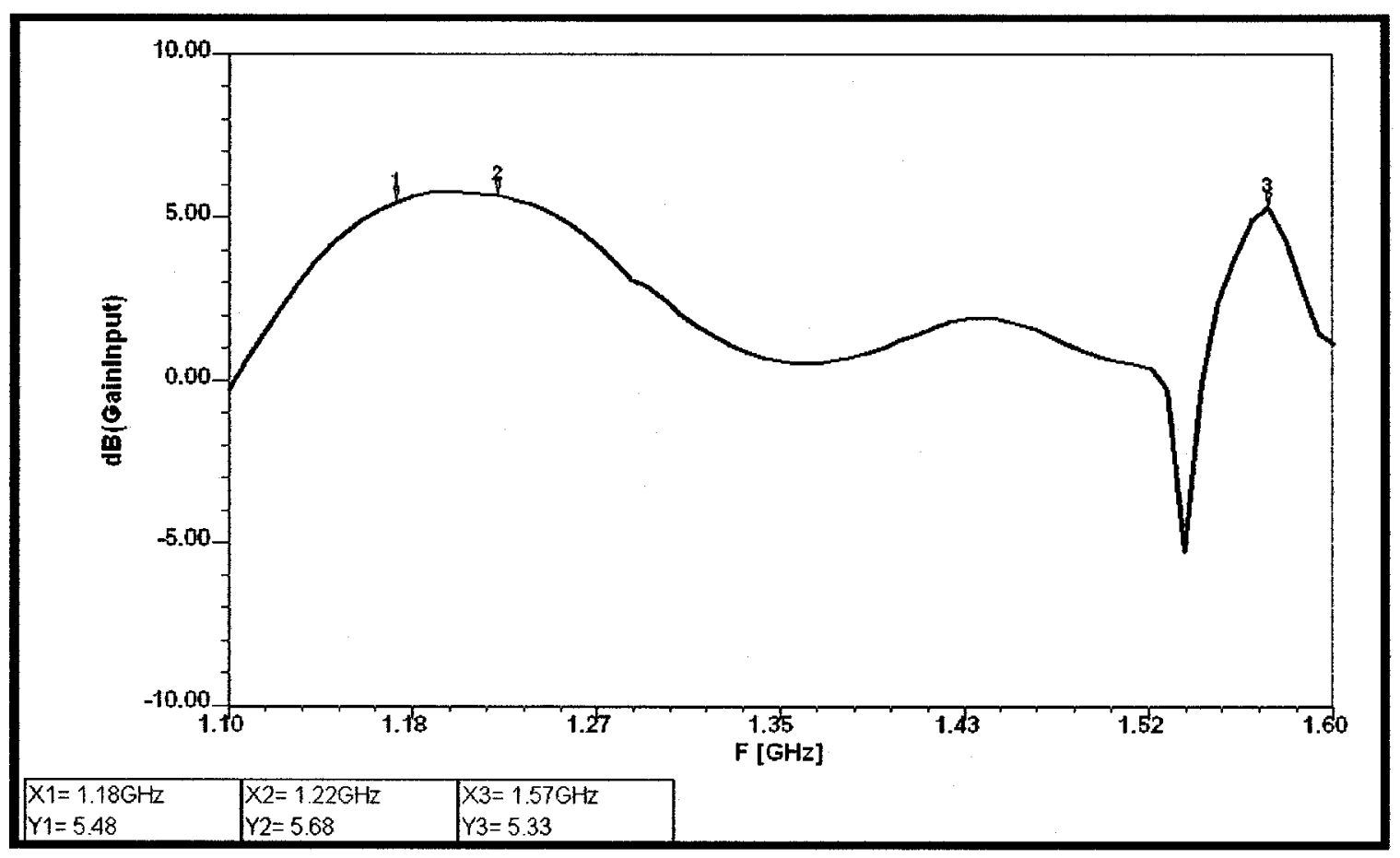

Figure 4.24: Simulated gain of the second antenna measured at the input port. [Ansoft Designer] 
Figure 4.25 shows the simulated axial ratio vs. frequency at broadside. It can be seen that an axial ratio of $1.60 \mathrm{~dB}$ at $\mathrm{L} 1,2.62 \mathrm{~dB}$ at $\mathrm{L} 2$, and $2.69 \mathrm{~dB}$ at $\mathrm{L} 5$ is achieved. Similar to the first design these simulated axial ratios do not take into account the three $100 \Omega$ resistors, which will reduce these values significantly. Also it can be seen that at the aperture resonant frequency of $1.53 \mathrm{GHz}$, the highest axial ratio is achieved. Figure 4.26 (a) through (c) shows the axial ratio vs. different theta angles in $\phi=0^{\circ}$ plane for the three desired frequencies. It can be seen that the axial ratio provides acceptable CP over the beamwidth of $90^{\circ}$ for $\mathrm{L} 1,130^{\circ}$ for $\mathrm{L} 2$, and $140^{\circ}$ for $\mathrm{L} 5$.

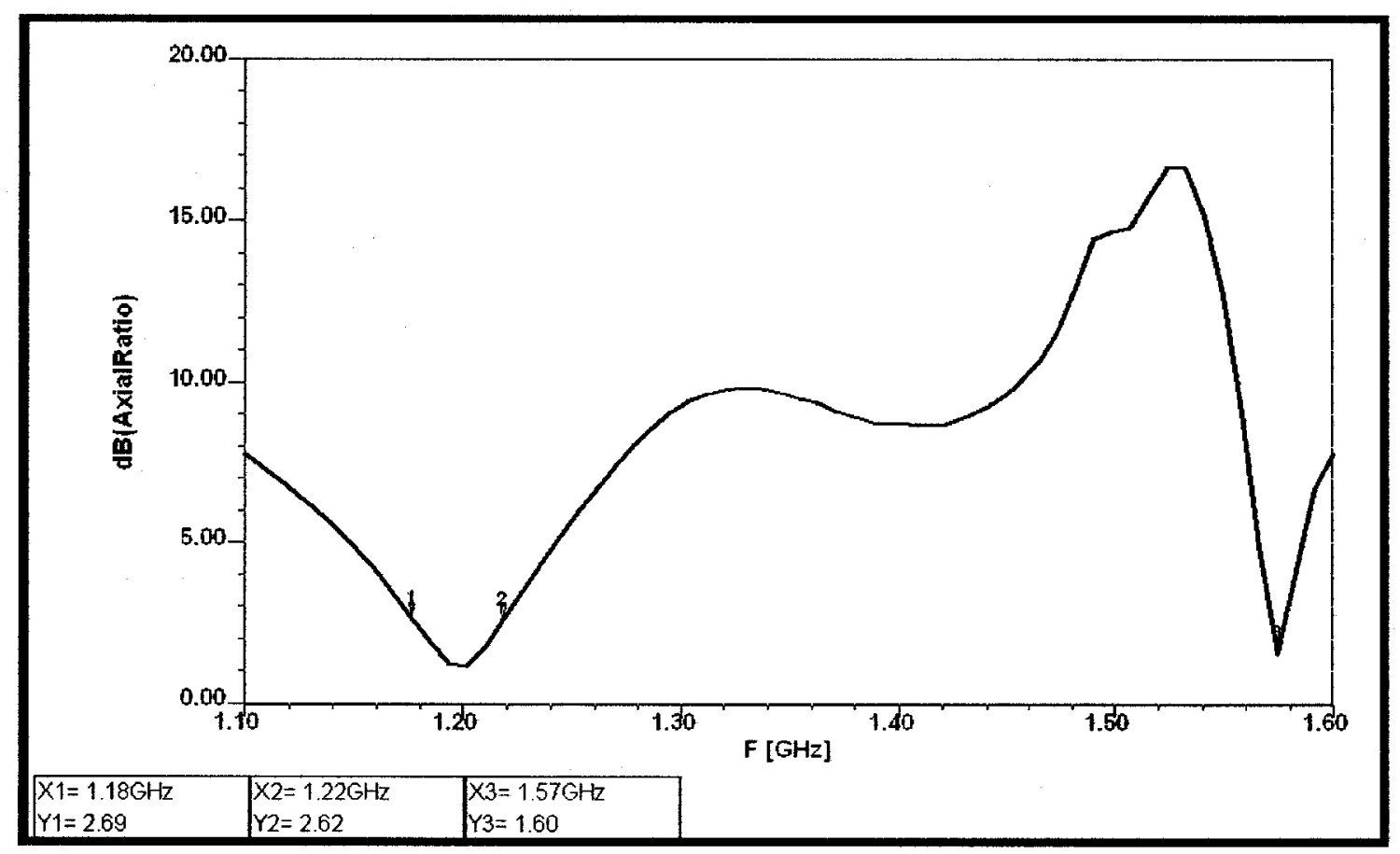

Figure 4.25: Simulated axial ratio vs. frequency of the second design at broadside. [Ansoft Designer] 


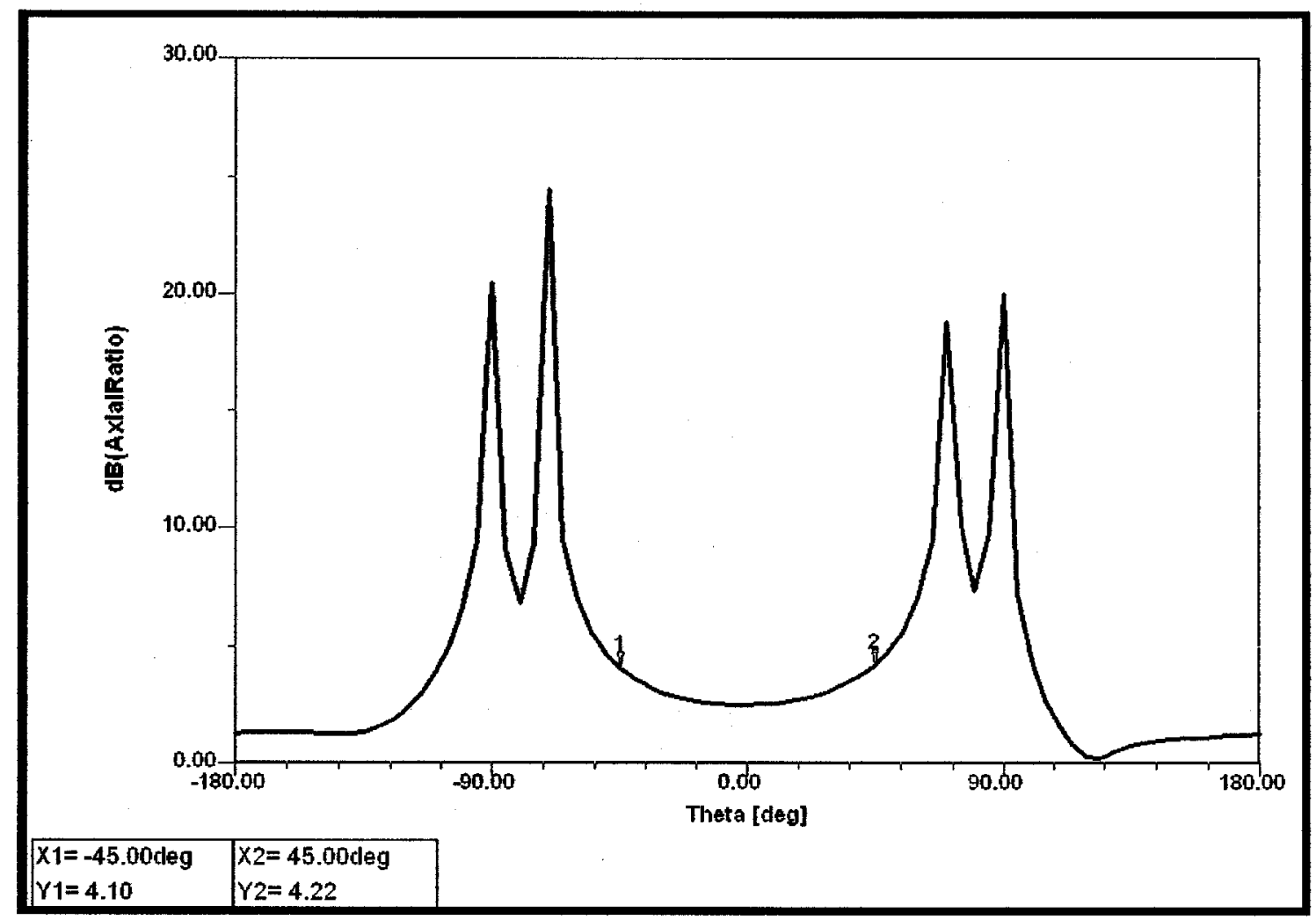

(a)

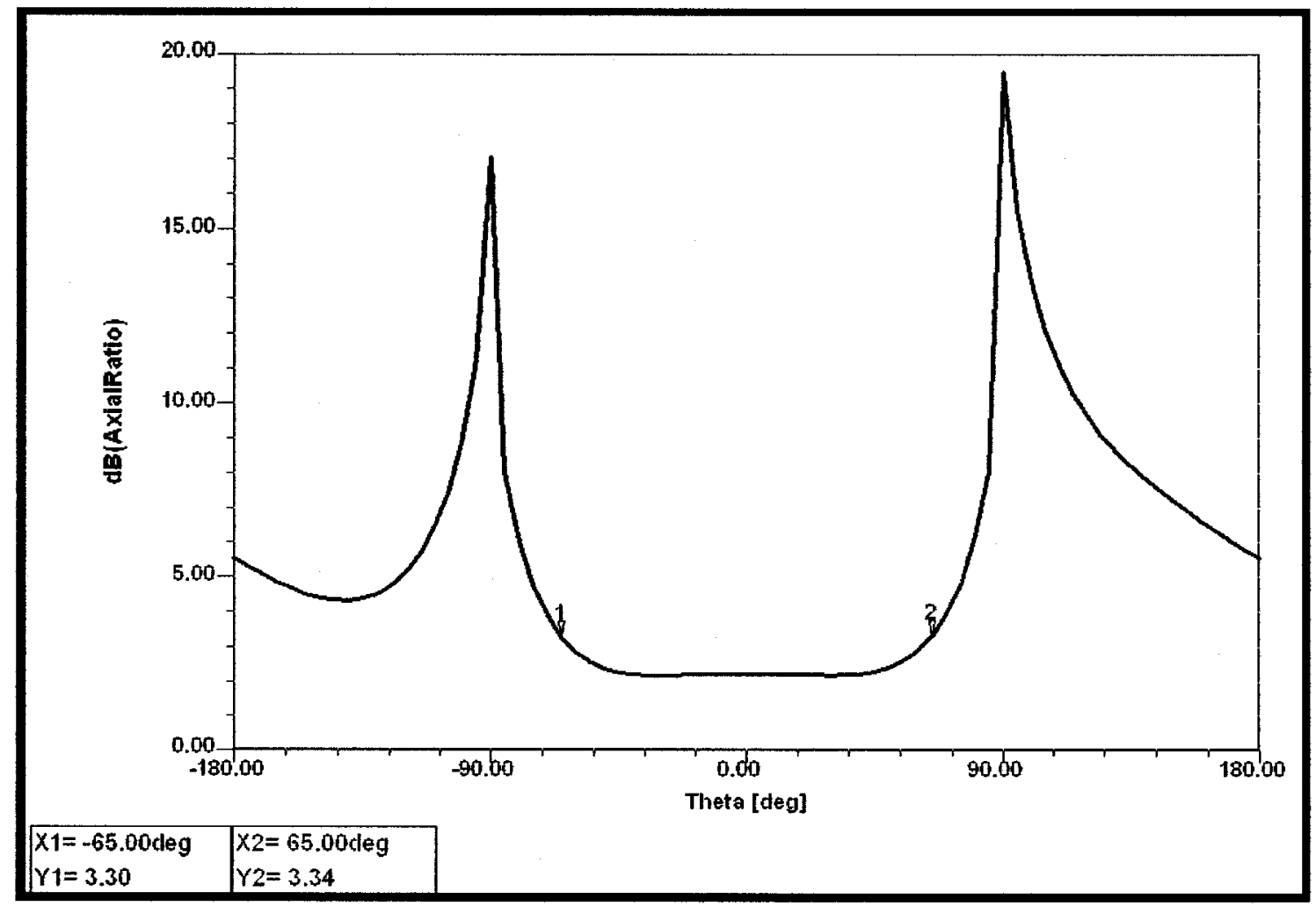

(b) 


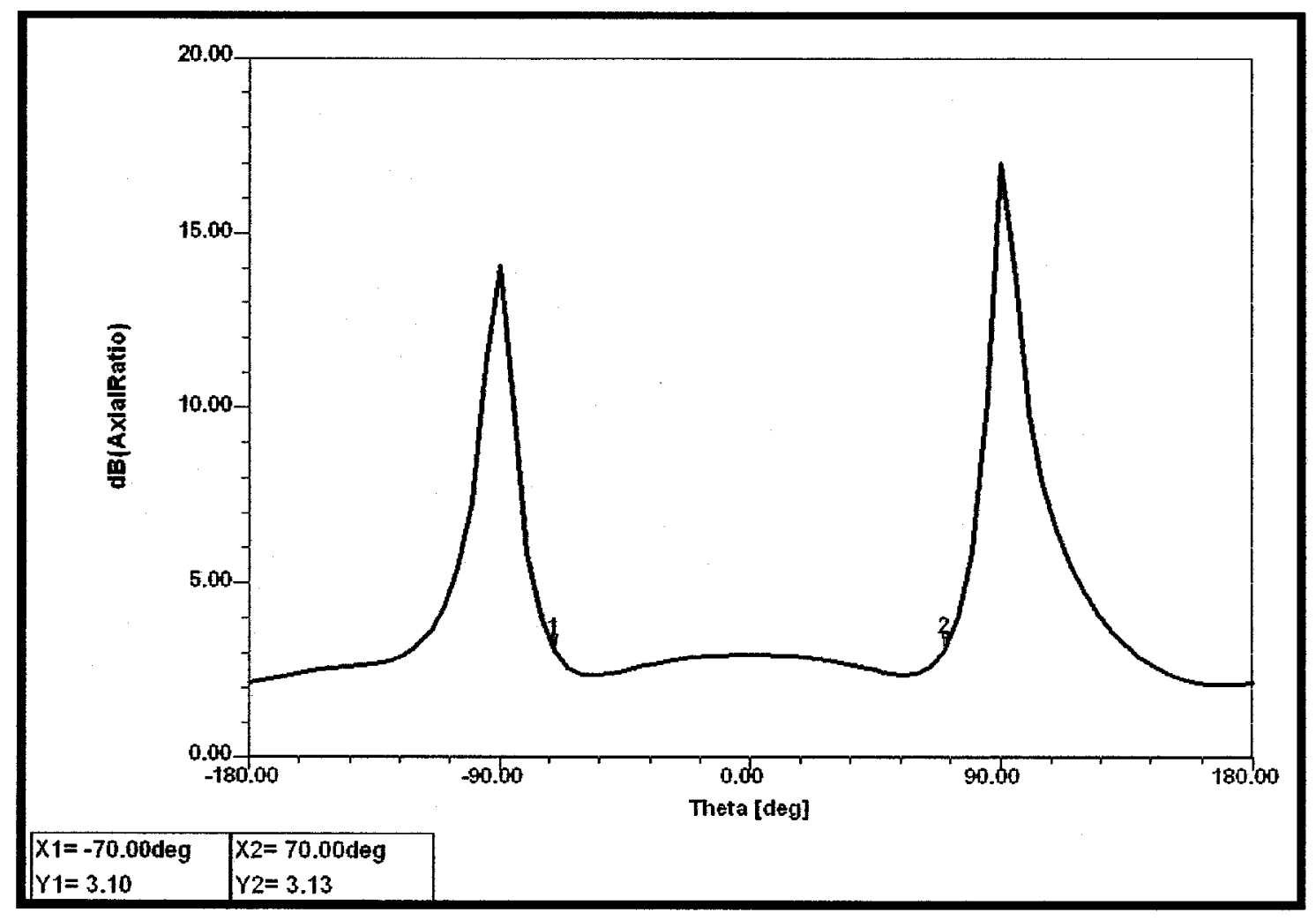

(c)

Figure 4.26: Simulated axial ratio vs. theta in $\phi=0^{\circ}$ plane.

(a) L1, (b) L2, (c) L5. [Ansoft Designer] 
Figure 4.27 (a) and (b) show the radiation patterns of the second designed antenna at $\mathrm{L} 1$, in the $\phi=0^{\circ}$ and $\phi=90^{\circ}$ planes respectively. It can be seen that at broadside a gain of $5.33 \mathrm{~dB}$ is achieved. Other characteristics of these patterns are: HPBW of about $72^{\circ}$, FNBW of $180^{\circ}$, and FBR of $6 \mathrm{~dB}$. Since this frequency band is somewhat close to the aperture resonance frequency of $1.53 \mathrm{GHz}$, the antenna performance has suffered from back lobe radiation of a maximum of $-1 \mathrm{~dB}$. This is slightly more than the first antenna since the aperture resonance is closer to the L1 band. This back lobe radiation can be improved by shielding the back of the antenna by another ground plane.

Figure 4.28 (a) and (b) show the radiation patterns at $\mathrm{L} 2$, in the $\phi=0^{\circ}$ and $\phi=$ $90^{\circ}$ planes respectively. It can be seen that a gain of $5.68 \mathrm{~dB}$ at broadside is achieved. Other characteristics of these patterns are: HPBW of about $125^{\circ}$, FNBW of $180^{\circ}$, and a FBR of $23 \mathrm{~dB}$. The FBR has improved significantly at this frequency.

Figure 4.29 (a) and (b) show the radiation patterns at L5, in the $\phi=0^{\circ}$ and $\phi=$ $90^{\circ}$ planes respectively. It can be seen that at broadside a gain of $5.58 \mathrm{~dB}$ is achieved. Other characteristics of these patterns are: HPBW of about $115^{\circ}, \mathrm{FNBW}$ of $180^{\circ}$, and a FBR of $16 \mathrm{~dB}$. The $3 \mathrm{D}$ radiation patterns of this antenna look very similar to the first antenna, therefore are not included to avoid repetition. All the radiation patterns at the three desired frequencies provide sharp nulls at low elevation angles, which make this antenna suitable for FRPA anti-jamming technique. The null positions are expected to shift from $\theta=90^{\circ}$, since the simulation method considers infinite substrate and ground plane. 


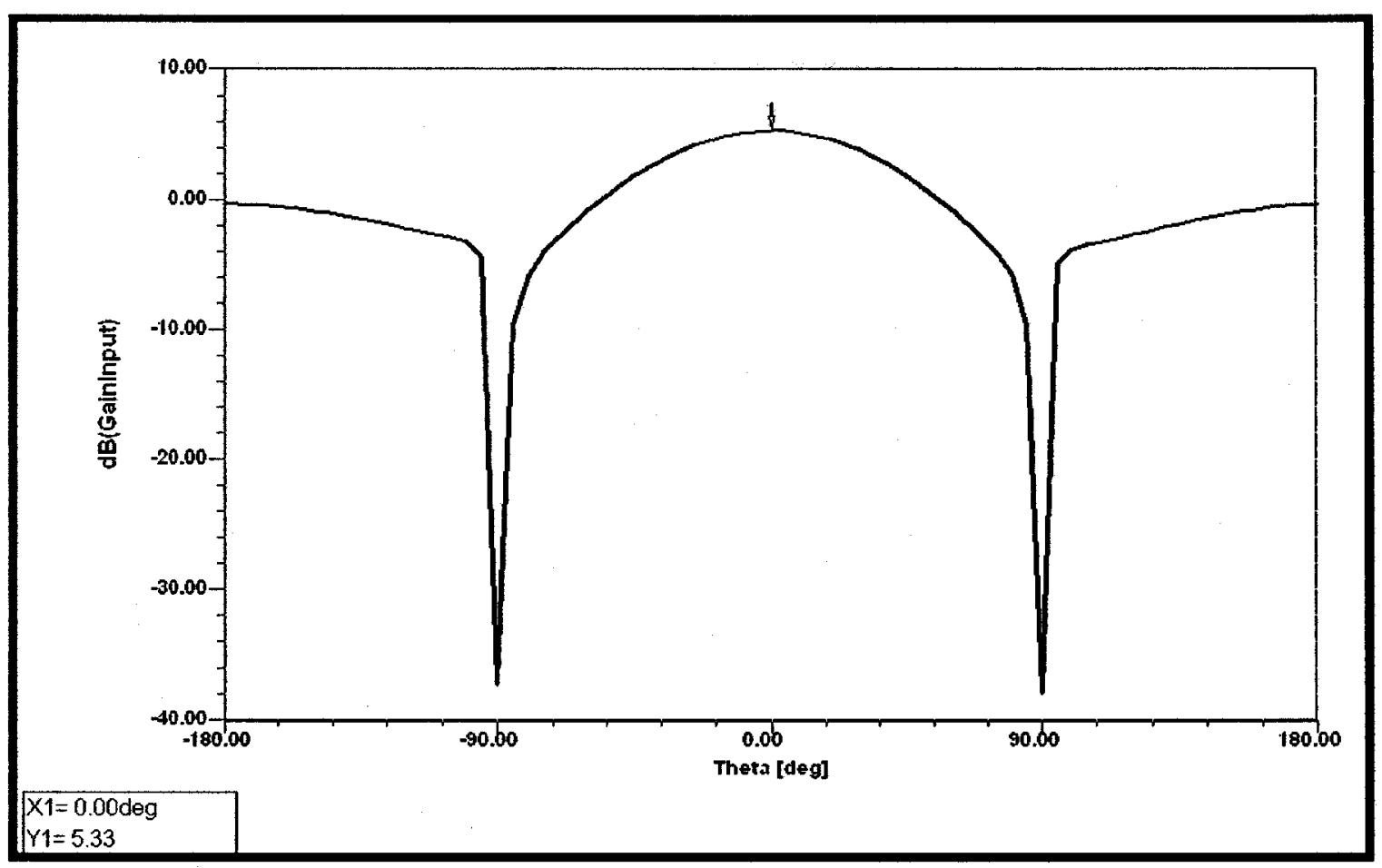

(a)

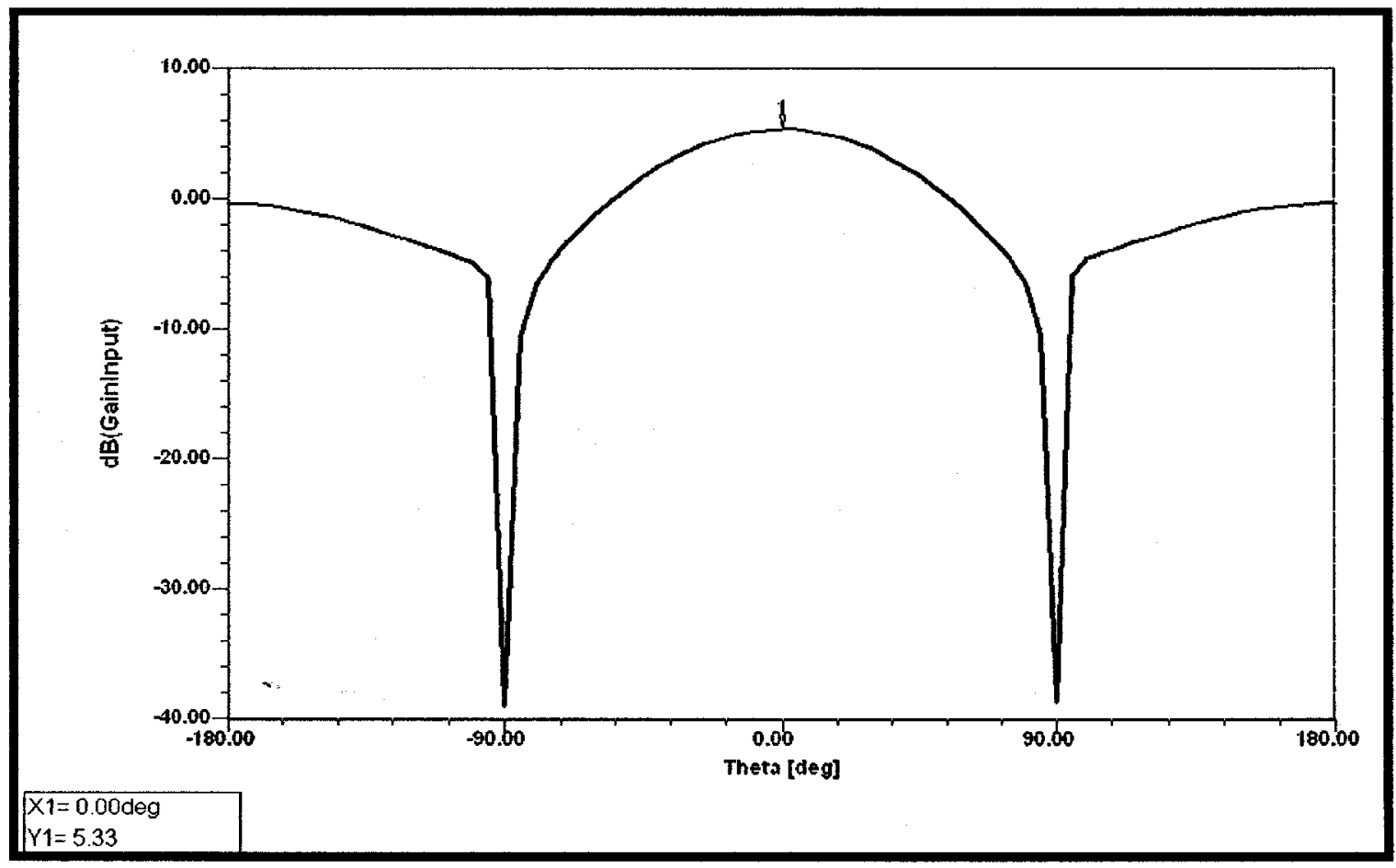

(b)

Figure 4.27: Simulated radiation pattern for $L 1$;

(a) $\phi=0^{\circ}$, (b) $\phi=90^{\circ}$. [Ansoft Designer] 


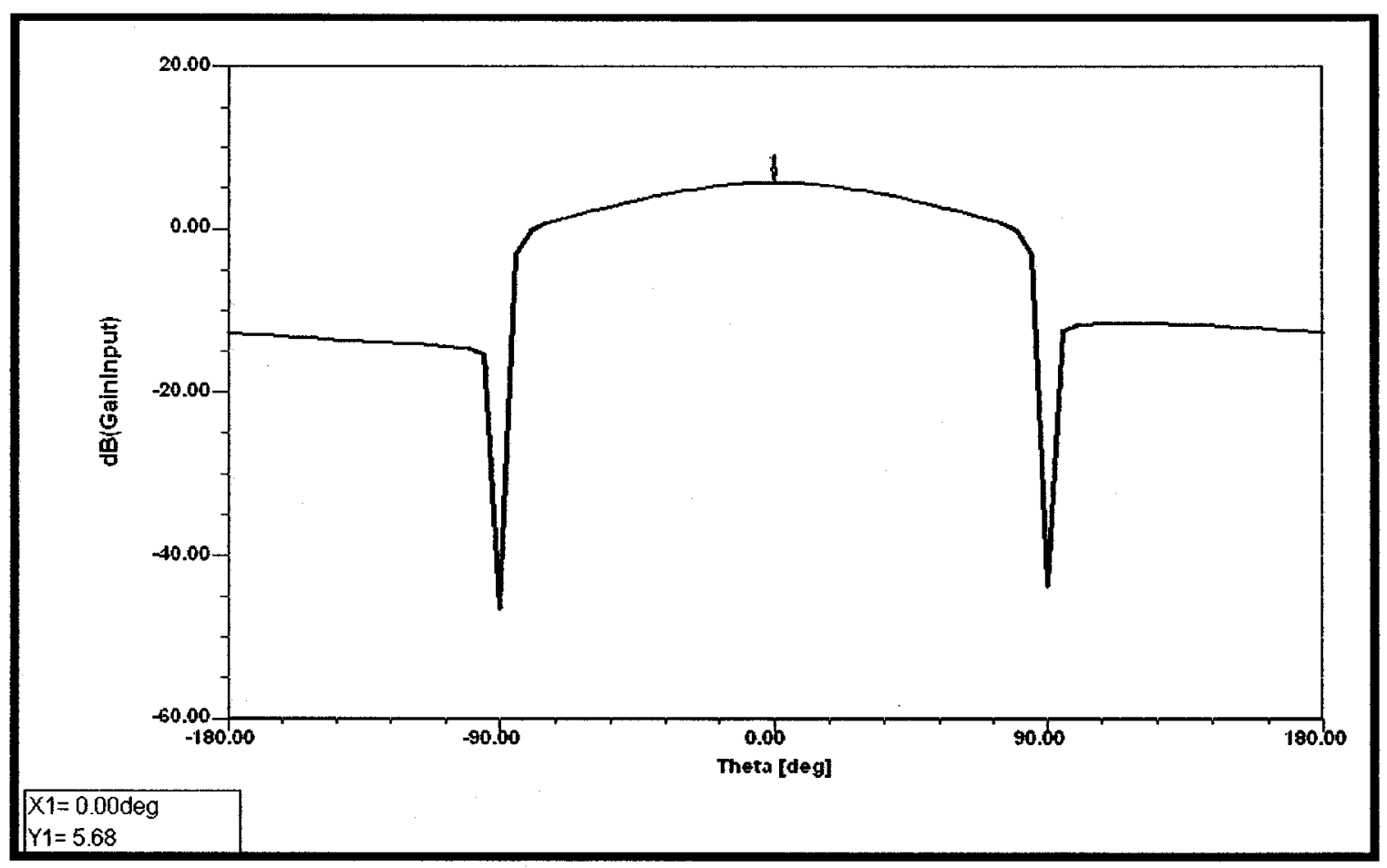

(a)

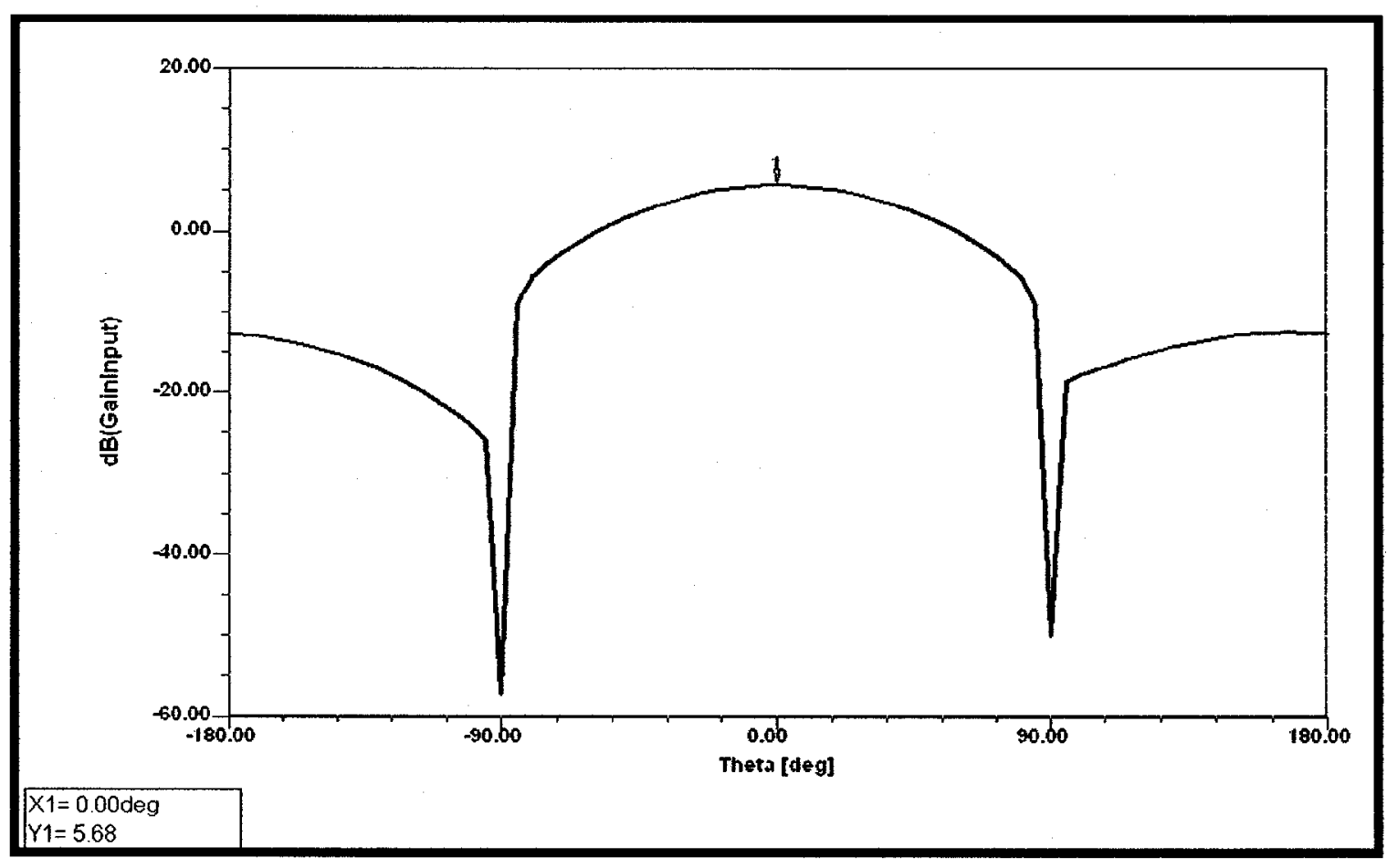

(b)

Figure 4.28: Simulated radiation pattern for $\mathrm{L} 2$;

(a) $\phi=0^{\circ}$, (b) $\phi=90^{\circ}$. [Ansoft Designer] 


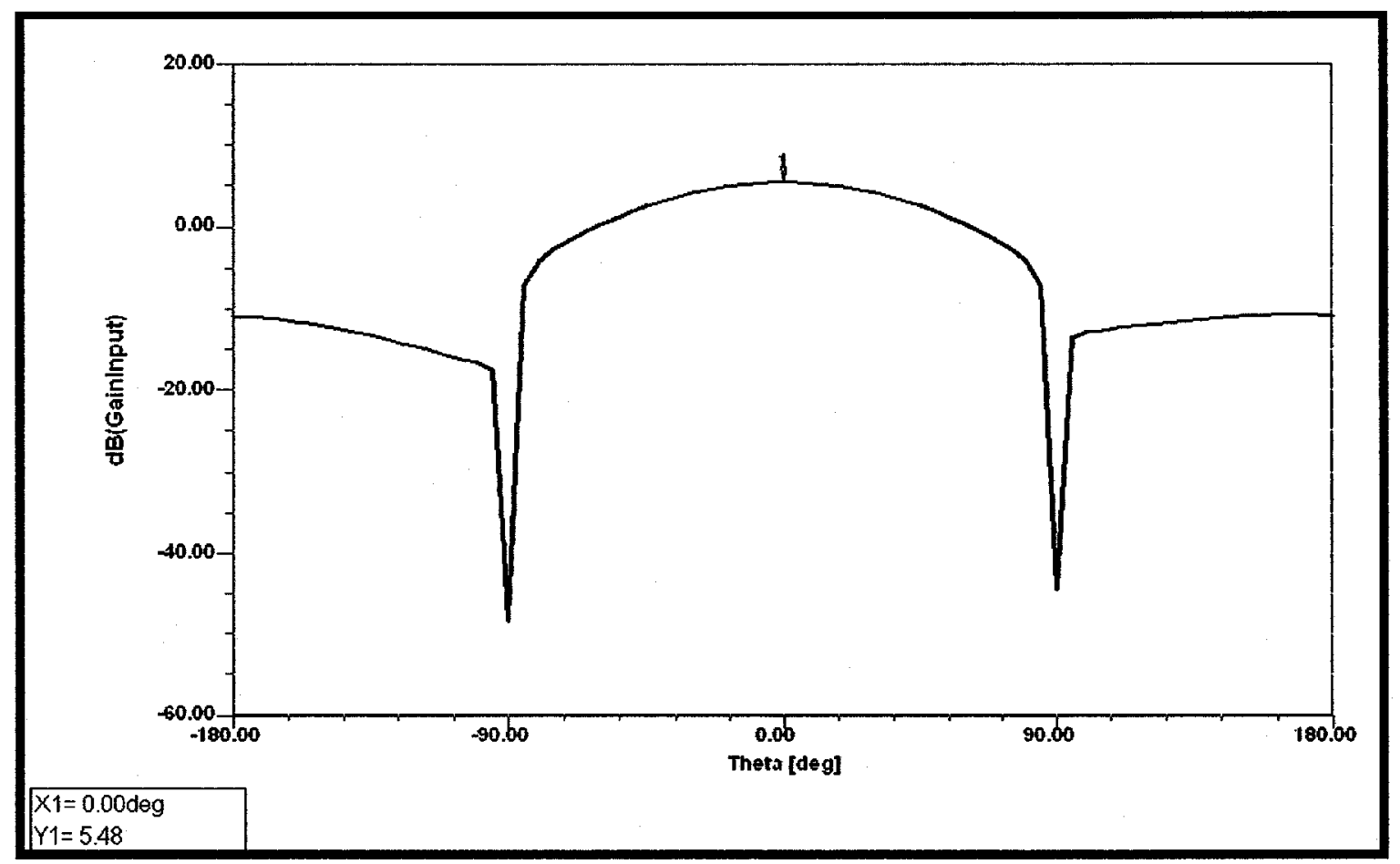

(a)

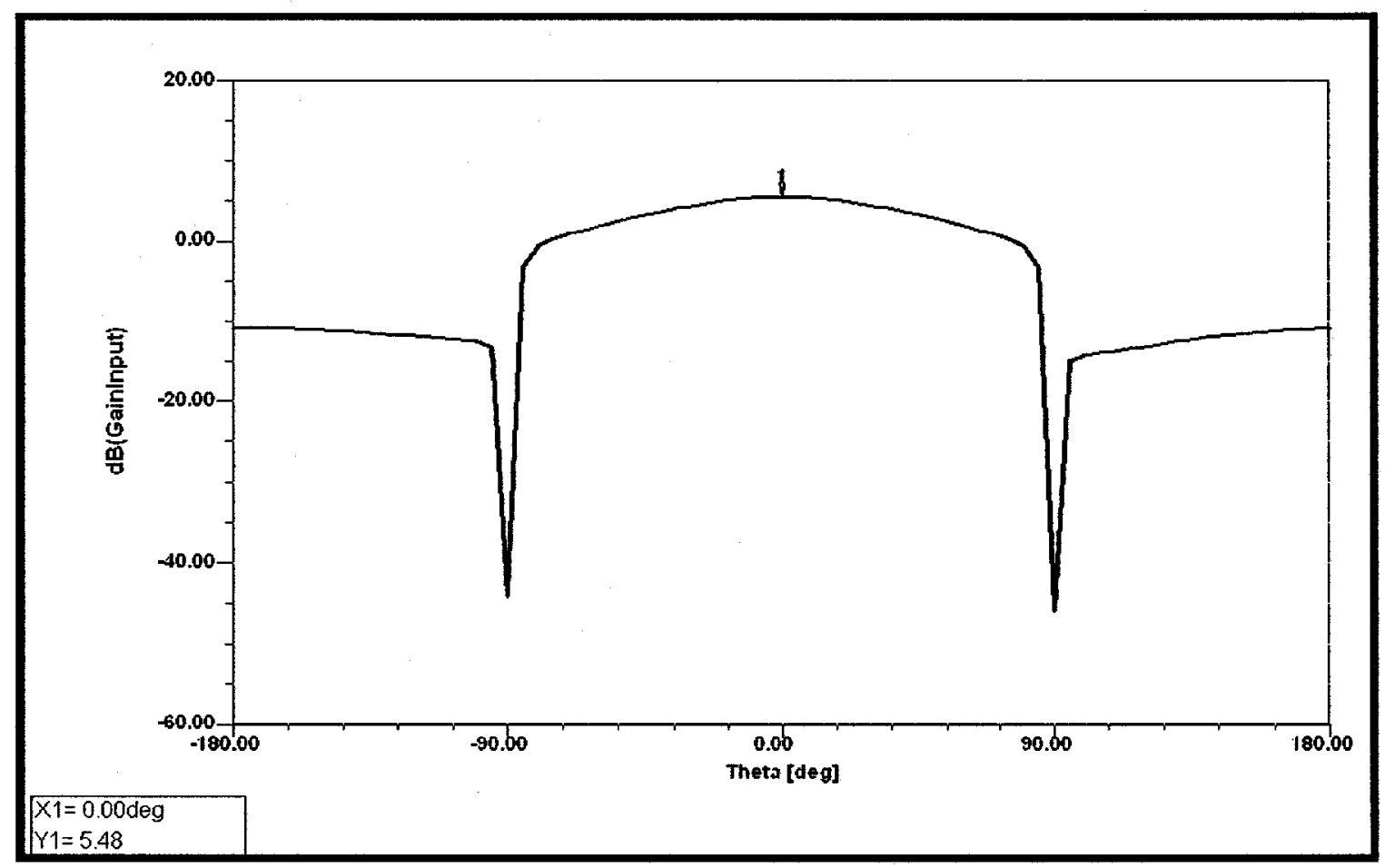

(b)

Figure 4.29: Simulated radiation pattern for L5;

(a) $\phi=0^{\circ}$, (b) $\phi=90^{\circ}$. [Ansoft Designer] 


\subsection{Conclusion:}

This chapter has presented the design procedure for an aperture-coupled stacked patch antenna. It was shown that due to numerous interacting design parameters there is no step-by-step design procedue for this structure; but by understanding the effect of each parameter on coupling and impedance loops, the design can become faster and less tedious. The parrallel feed configuration was described in detail and provides accurate phase and amplitude for circular polarization and signal combining. The design procedure of two dual-band aperture-coupled stacked patch antenna in GPS frequencies L1 and L2/L5 was included. This procedure can be summarized as follows:

- Choose the patch and the feed substrate materials.

- Start with the linear polarized configuration shown in Figure 4.1. In this step the goal is to determine the two patch sizes, the substrate thicknesses, and the aperture length. These dimensions remain almost the same for the circular polarized design. The initial patch dimensions can be estimated using equations provided in Chapter 3.

- Design the feed network so that power is divided properly between the four feedlines. This step ensures proper circular polarization. The Wilkinson power combiners must be designed with especial care since power imbalance between the two input legs degrade the axial ratio and can be seen in Figure 4.9. The lengths of the tuning stubs are another important factor, which controls the frequency of operation.

- The cross aperture is placed in the common ground plane. The aperture length found in the linear polarized design is slightly increased. 
These two similar structures were both designed and simulated in Ansoft designer. The simulated results satisfy the design requirements such as impedance bandwidth, circular polarization, and gain for the three desired GPS frequencies. The next chapter presents the fabrication and test results of the two antennas. 


\section{ChaPTer 5}

\section{Fabrication And Testing}

\subsection{Fabrication}

The antennas were fabricated in the CRC fabrication shop. Here's a summary of the etching process:

1. At first a photo mask is created that covers the wanted copper tracing.

2. The masked substrates are inserted into a chamber in which is heated and sprayed with an ammonia based chemical. At this step the unwanted copper is removed.

3. After each layer is properly etched, these layers are bonded. For the first antenna the Rohacell foam and FR4 material were bonded using a cement glue spray. The second antenna bonding was done with Arlon CLPE-P bonding sheets. This very thin layer $(0.08 \mathrm{~mm})$ has the same permittivity as the Arlon CLTE and is heated to about $300^{\circ} \mathrm{C}$. Unfortunately due to the difference in coefficient of thermal expansion of Rogers TMM10i and Arlon CLTE this process failed to cool down, and the Rogers TMM10i layer cracked. Therefore bonding was done by screwing the layers together.

4. The resistors and an SMA connector are soldered to the feed line.

Figures 5.1 and 5.2 show the fabricated antennas. 


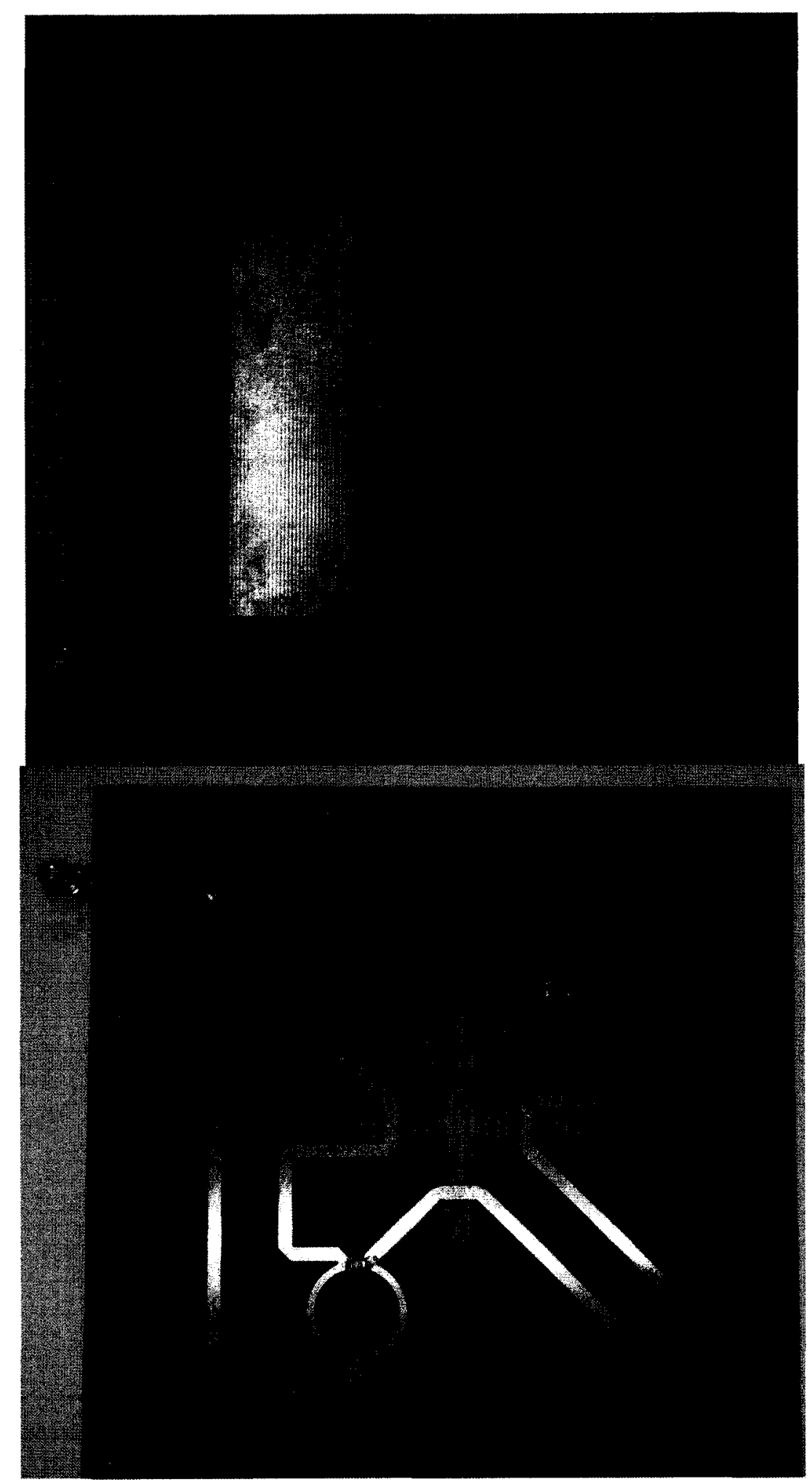

Figure 5.1: Fabricated aperture-coupled stacked patch antenna (Antenna 1) 


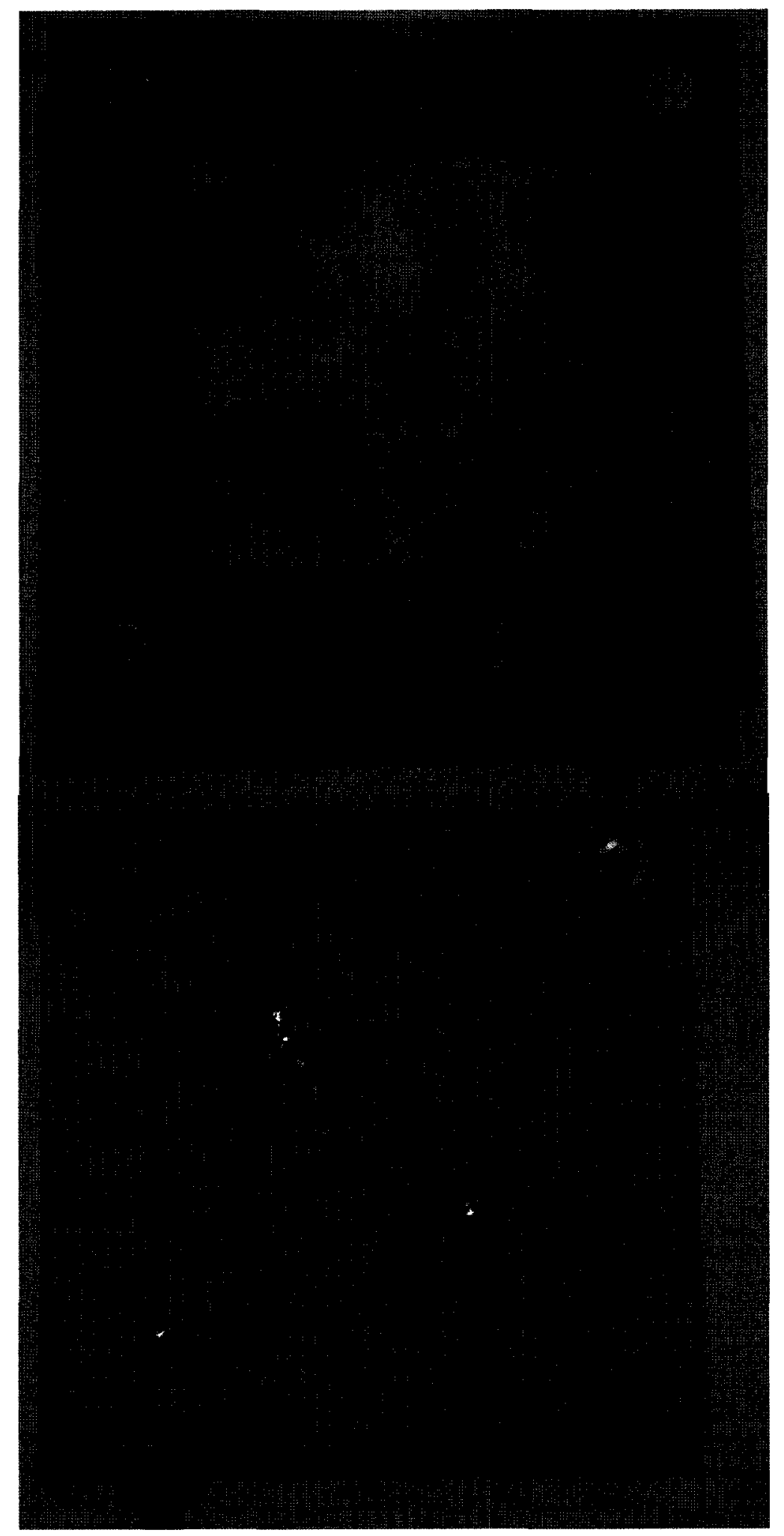

Figure 5.2: Fabricated aperture-coupled stacked patch antenna (Antenna 2) 


\subsection{Testing}

Both antennas were tested for their return loss and radiation patterns. The following results were obtained at the Carleton university microwave lab and anechoic chamber. A summary of measurement setup is presented in the Appendix A.

\subsubsection{First Antenna Test Results}

Figure 5.3 shows the tested S11 for the first designed antenna. It can be seen that this antenna is matched at the three desired frequencies with return loss of $-16.7 \mathrm{~dB}$ at $\mathrm{L} 1$, $-25.5 \mathrm{~dB}$ at $\mathrm{L} 2$, and $-20.5 \mathrm{~dB}$ at L5. Also a bandwidth of $22 \mathrm{MHz}$ for $\mathrm{L} 1$, and $160 \mathrm{MHz}$ for L2/L5 is achieved.

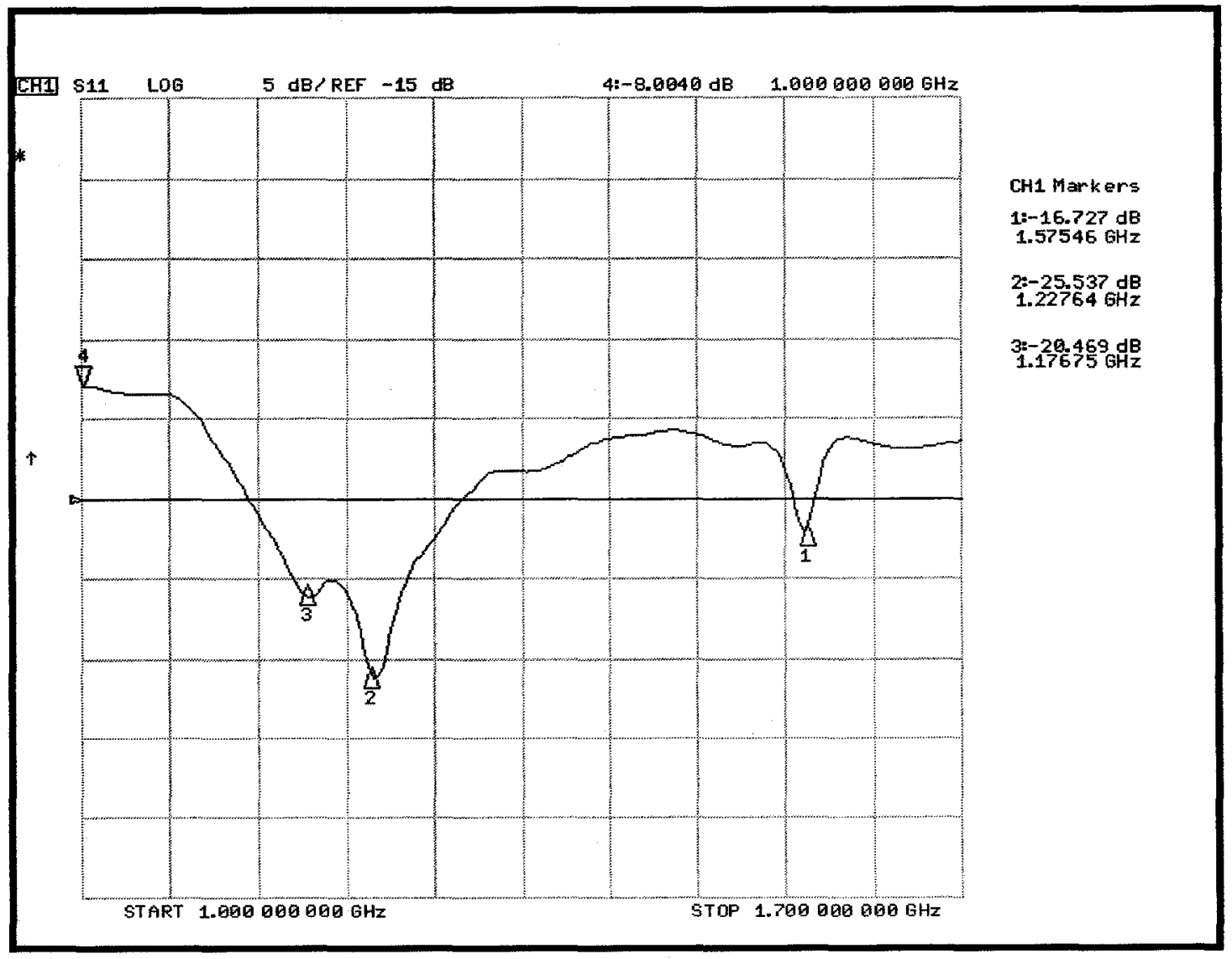

Figure 5.3: Tested return loss of the first antenna. [Agilent Network Analyzer] 
Figure 5.4 (a) and (b) show the radiation patterns of the first antenna at L1, in the $\phi=0^{\circ}$ and $\phi=90^{\circ}$ planes respectively. It can be seen that at broadside for $\phi=0^{\circ}$ a gain of $5.36 \mathrm{~dB}$ and for $\phi=90^{\circ}, 5.4 \mathrm{~dB}$ is achieved. Other characteristics of these patterns are: axial ratio of $1.5 \mathrm{~dB}$ at $\theta=0^{\circ}$, half power beamwidth (HPBW) of about $80^{\circ}$, null to null beamwidth (FNBW) of $170^{\circ}$, and a front to back ratio (FBR) of $7 \mathrm{~dB}$. Figure 5.5 shows the axial ratio vs. angle theta in $\phi=0^{\circ}$ plane. It can be seen that at L1, the antenna provides good CP over a beamwidth of $110^{\circ}$ (CPBW).

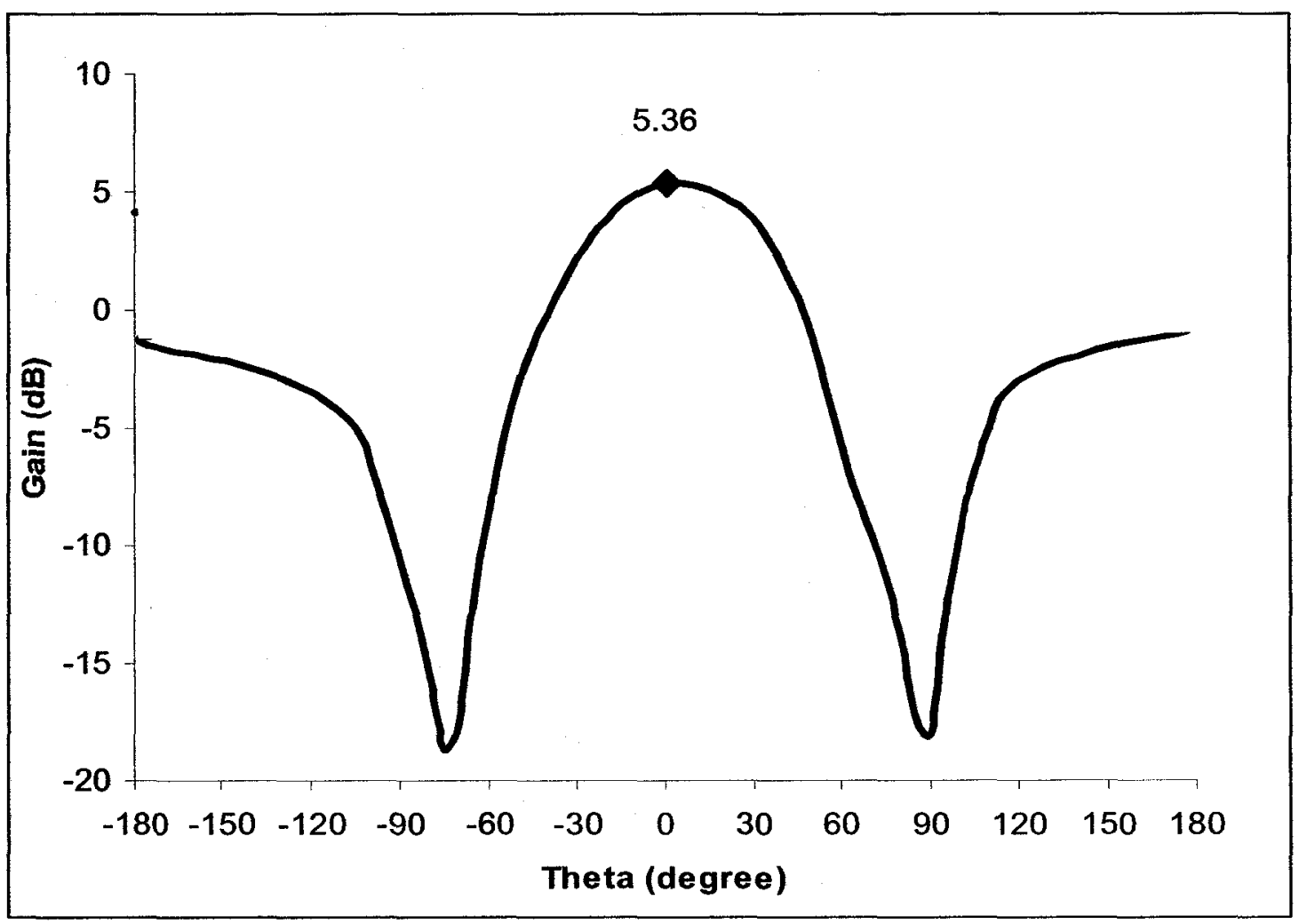

(a) 


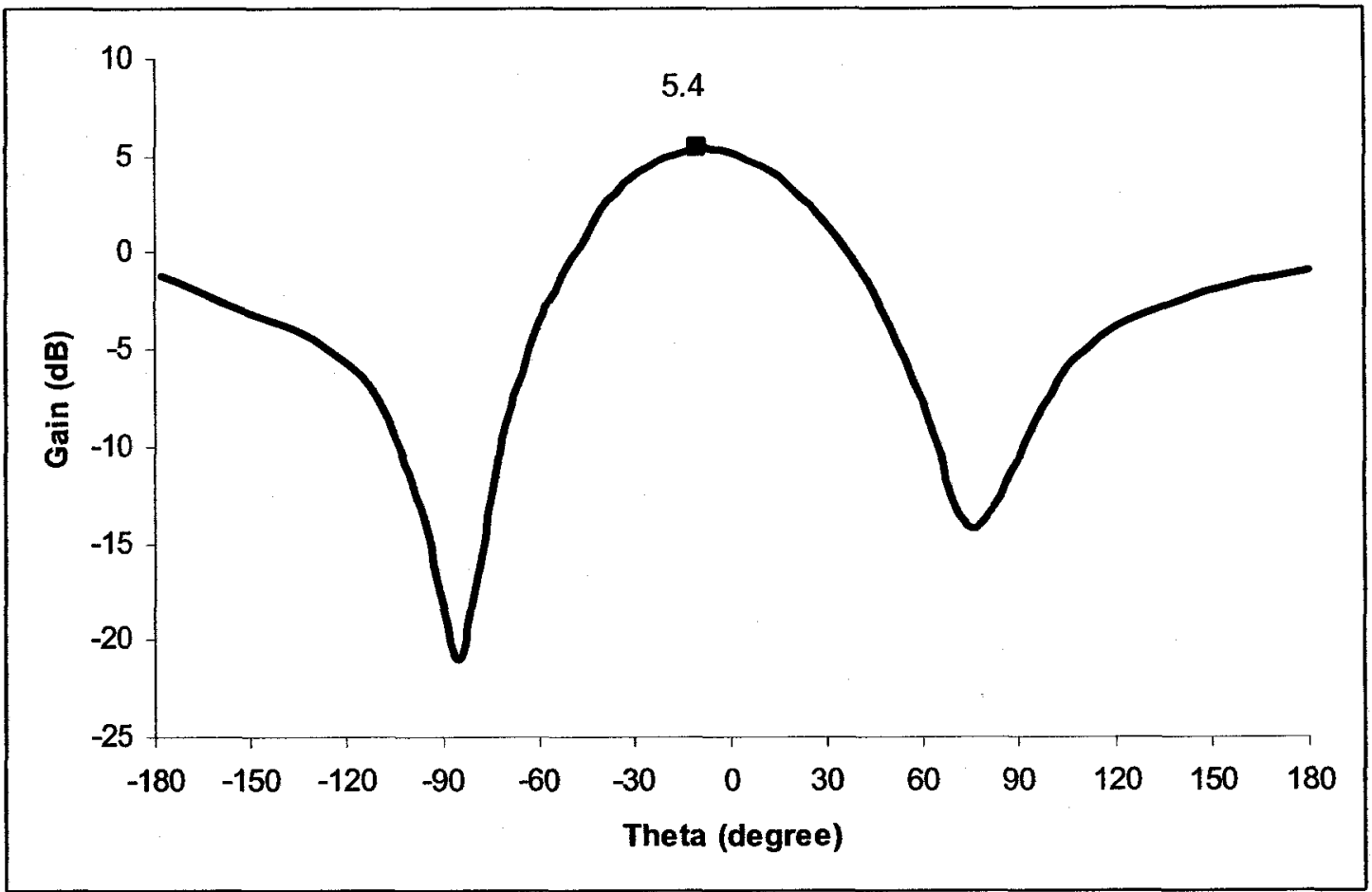

(b)

Figure 5.4: First Antenna radiation pattern for L1;

(a) $\phi=0^{\circ}$, (b) $\phi=90^{\circ}$.

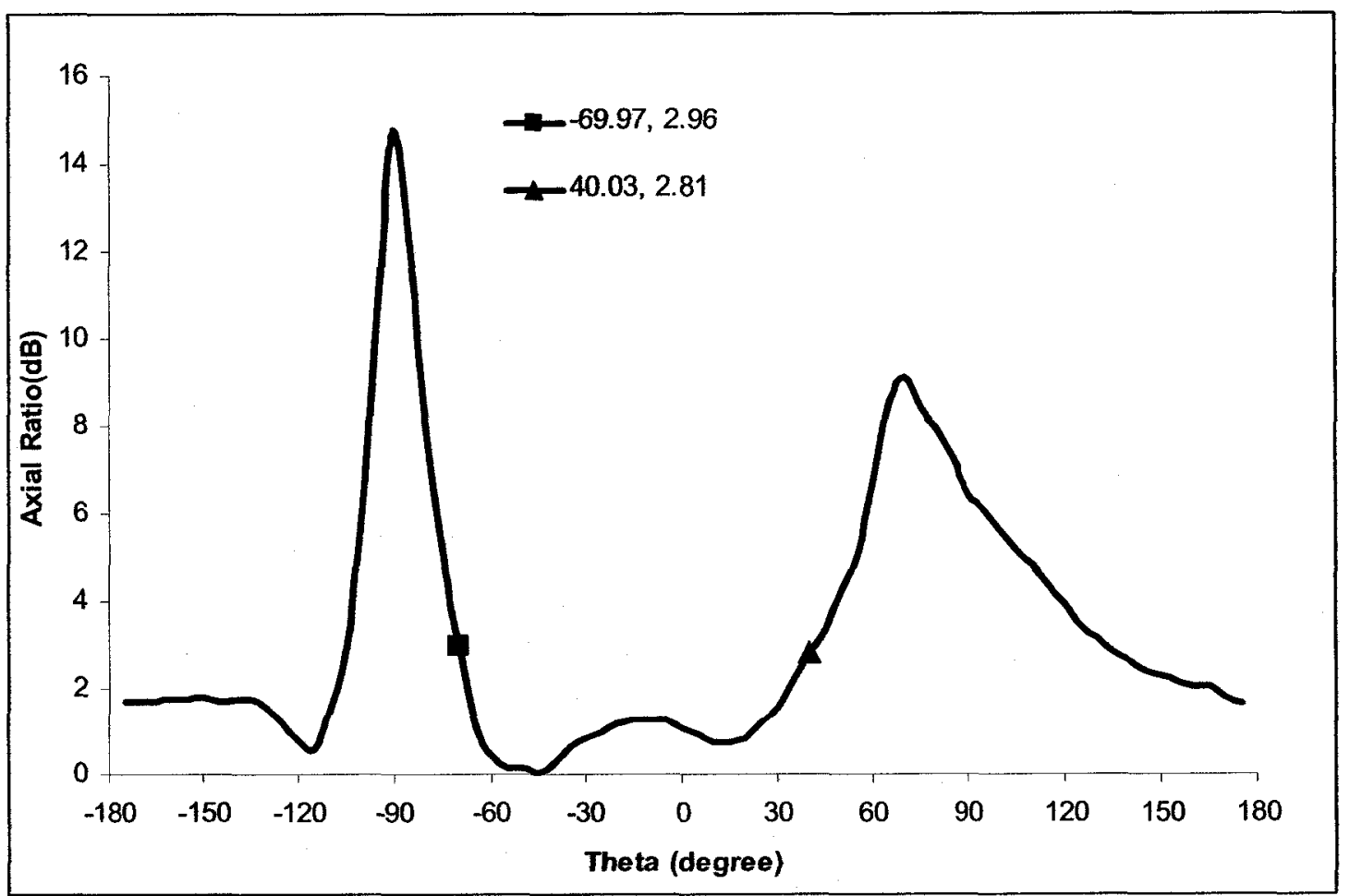

Figure 5.5: Axial ratio vs. theta in $\phi=0^{\circ}$ plane for L1. 
Figure 5.6 (a) and (b) show the radiation patterns of the first antenna at L2, in the $\phi=0^{\circ}$ and $\phi=90^{\circ}$ planes respectively. It can be seen that at broadside for $\phi=0^{\circ}$ a gain of $7.3 \mathrm{~dB}$ and for $\phi=90^{\circ}, 6.98 \mathrm{~dB}$ is achieved. Other characteristics of these patterns are: axial ratio of $1.7 \mathrm{~dB}$ at $\theta=0^{\circ}$, HPBW of about $75^{\circ}$, FNBW of $230^{\circ}$, and FBR of $15 \mathrm{~dB}$. Figure 5.7 shows the axial ratio vs. angle theta in $\phi=0^{\circ}$ plane. It can be seen that at L2, the antenna provides good CP over a beamwidth of $100^{\circ}$. Also the effect of finite size ground plane is visible in these patterns, which result in larger FNBW.

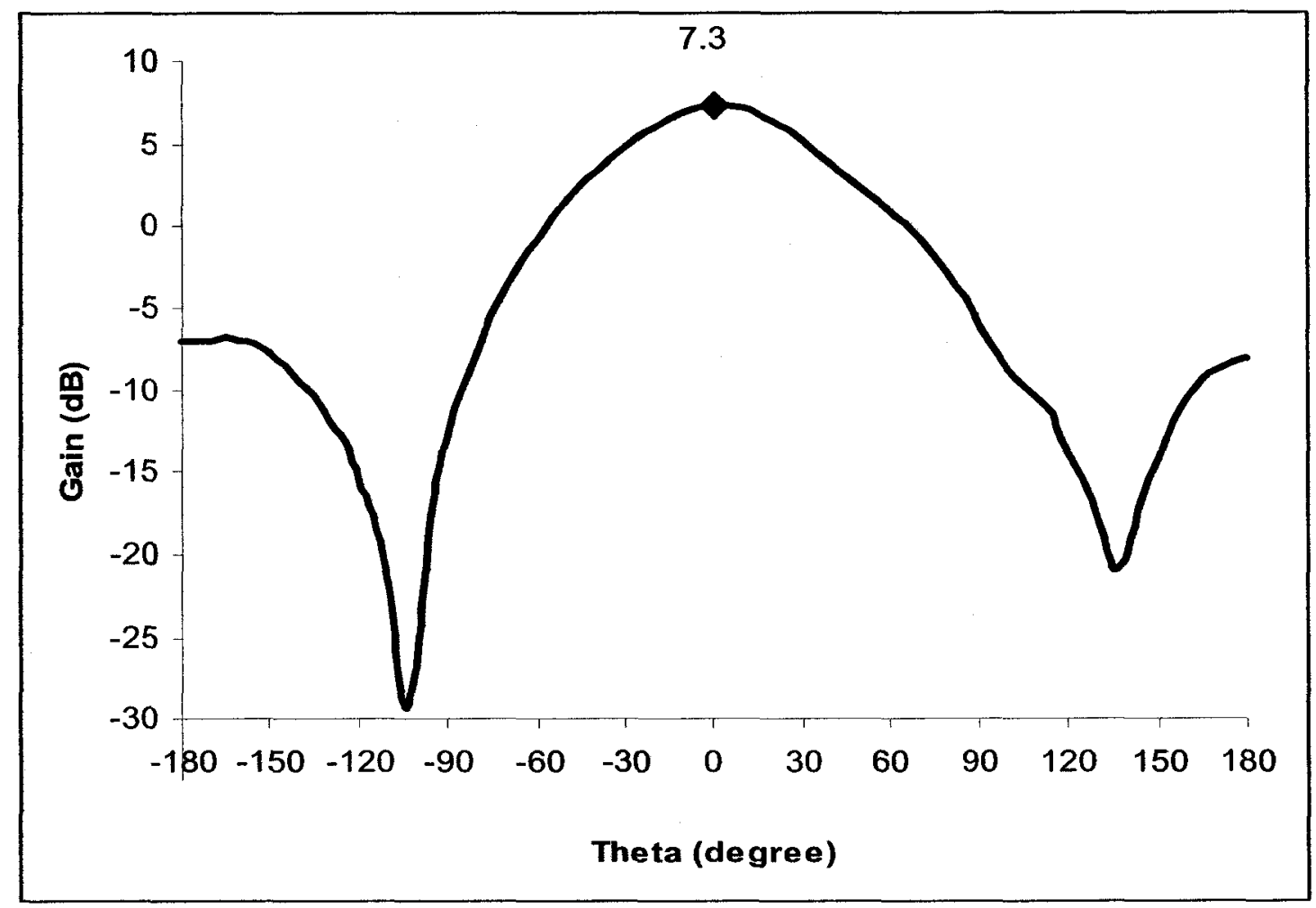

(a) 


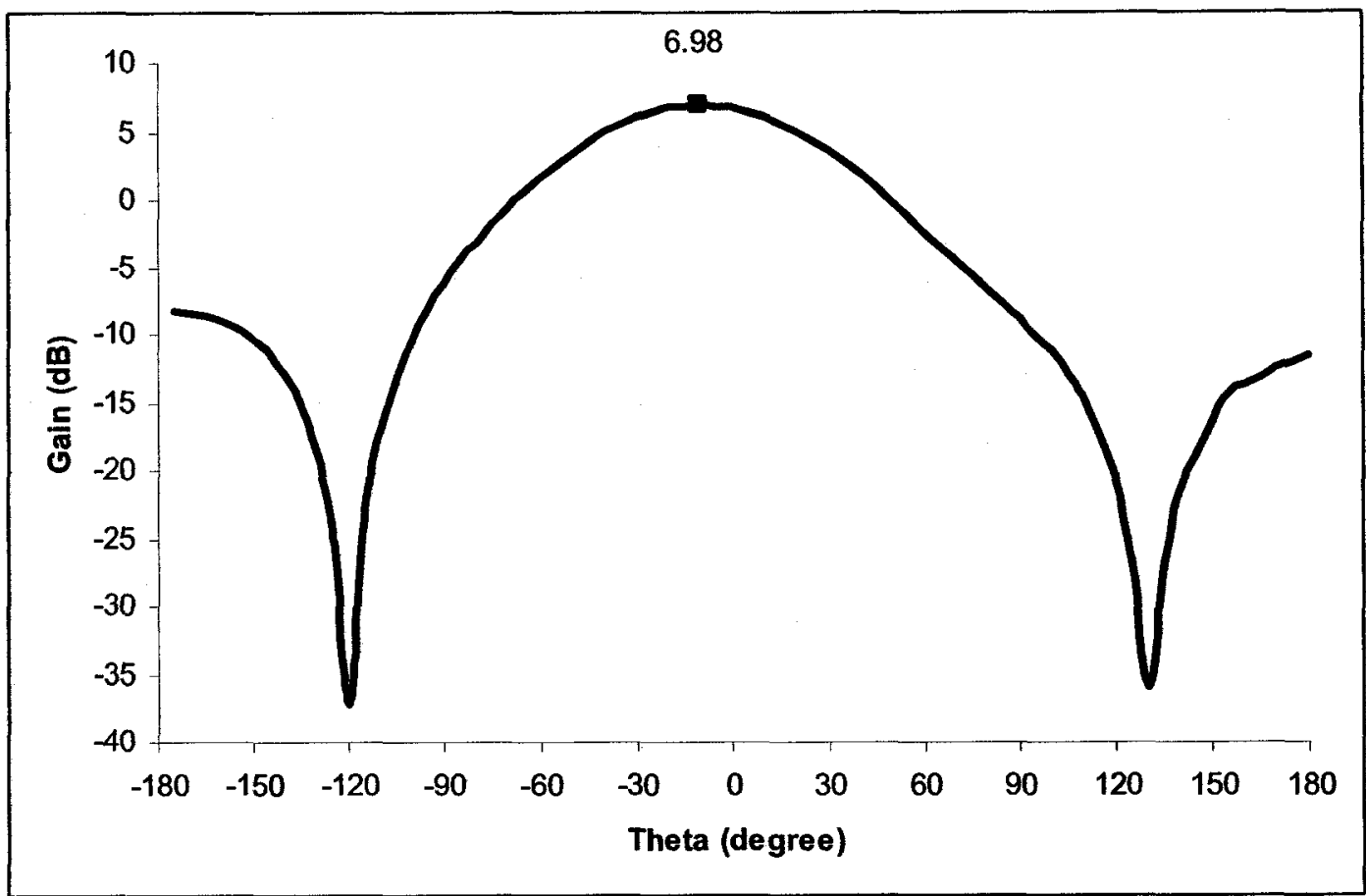

(b)

Figure 5.6: First Antenna radiation pattern for L2;

(a) $\phi=0^{\circ}$, (b) $\phi=90^{\circ}$.

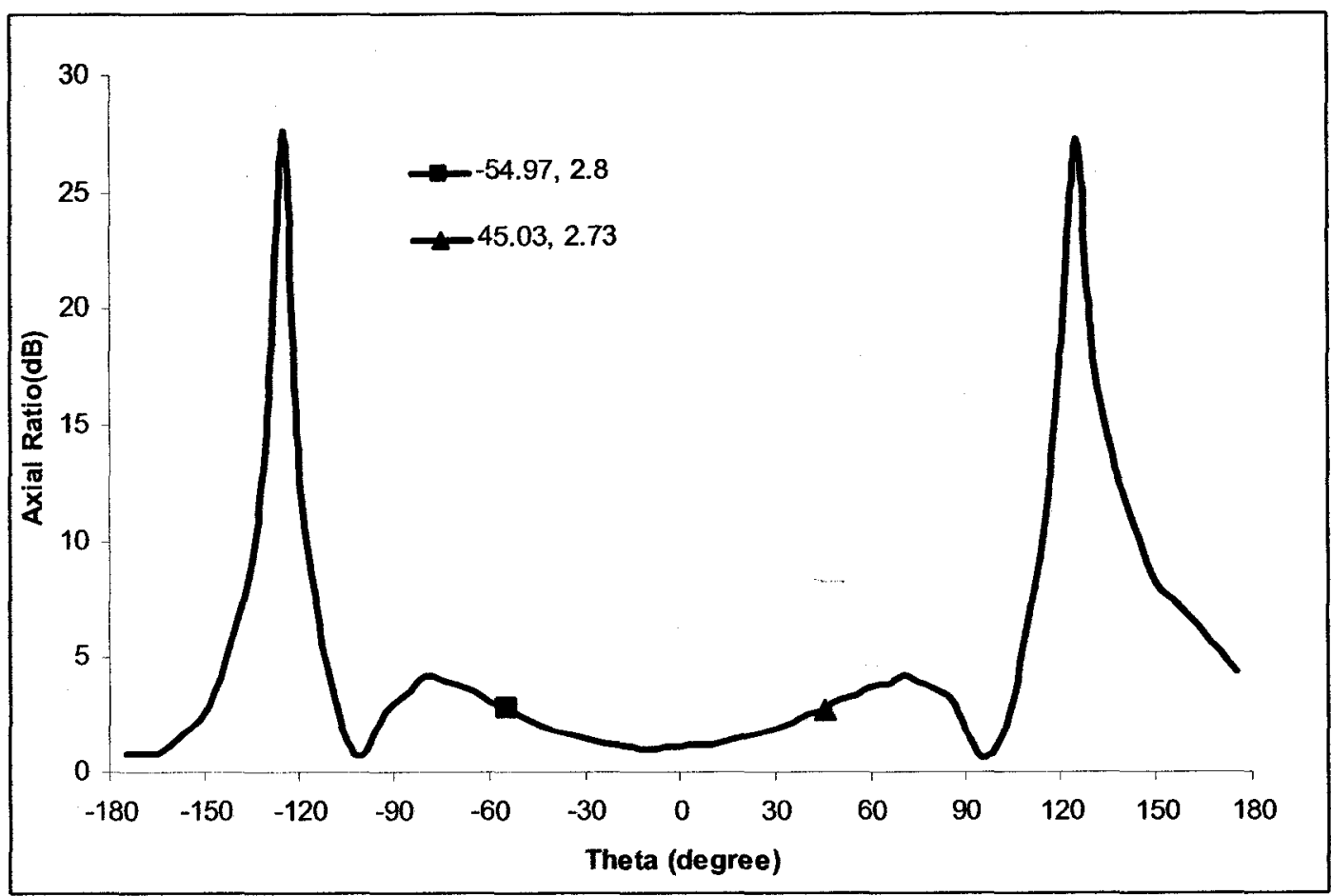

Figure 5.7: Axial ratio vs. theta in $\phi=0^{\circ}$ plane for $\mathrm{L} 2$. 
Figure 5.8 (a) and (b) show the radiation patterns of the first antenna at L5, in the $\phi=0^{\circ}$ and $\phi=90^{\circ}$ planes respectively. It can be seen that at broadside for $\phi=0^{\circ}$ a gain of $6.07 \mathrm{~dB}$ and for $\phi=90^{\circ}, 6.29 \mathrm{~dB}$ is achieved. Other characteristics of these patterns are: axial ratio of $1.5 \mathrm{~dB}$ at $\theta=0^{\circ}$, HPBW of about $90^{\circ}, \mathrm{FNBW}$ of $270^{\circ}$, and FBR of $14 \mathrm{~dB}$. Figure 5.9 shows the axial ratio vs. angle theta in $\phi=0^{\circ}$ plane. It can be seen that at L5, the antenna provides good CP over a beamwidth of $110^{\circ}$. Also the effect of finite size ground plane is visible in these patterns, which result in an increase in FNBW.

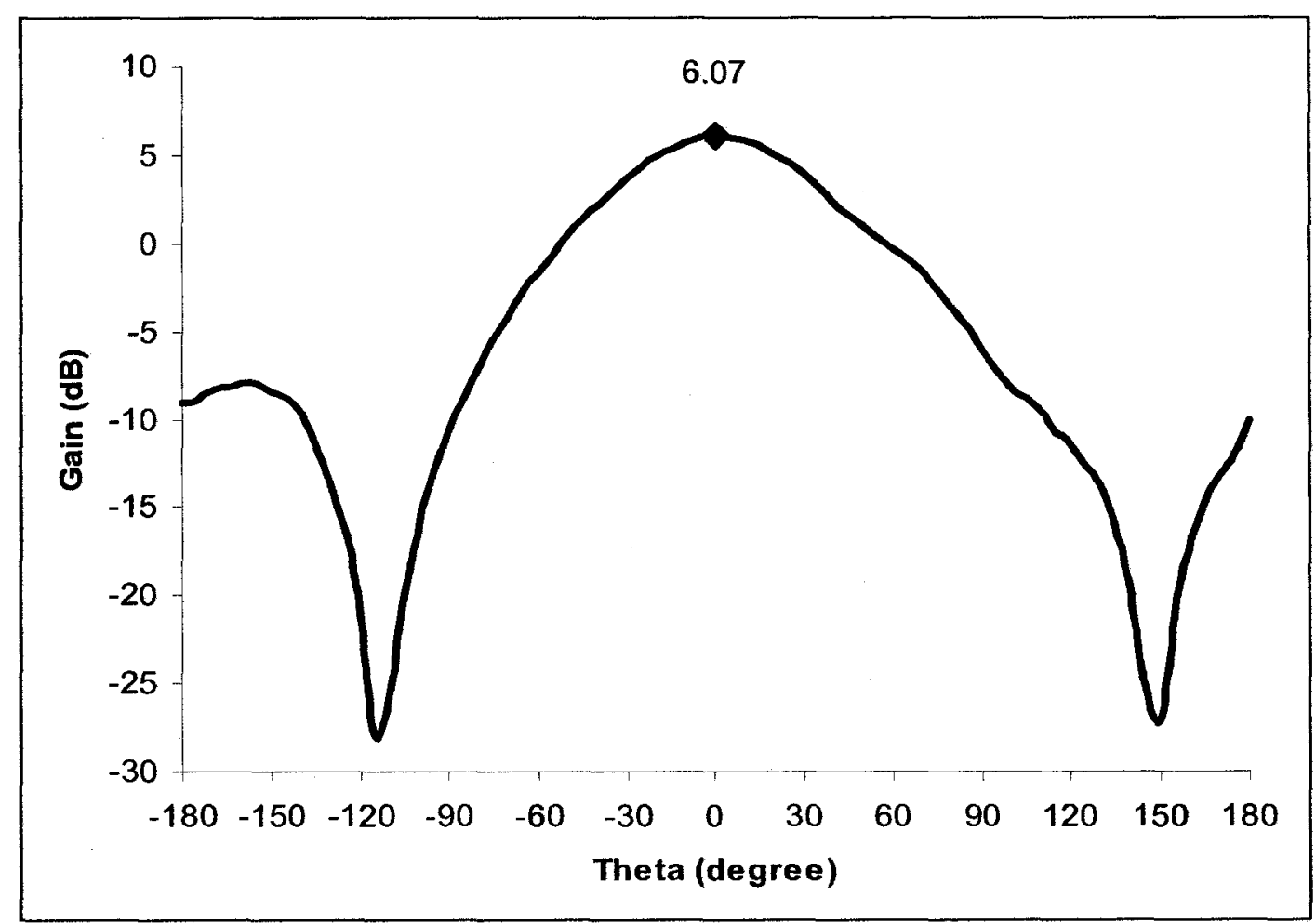

(a) 


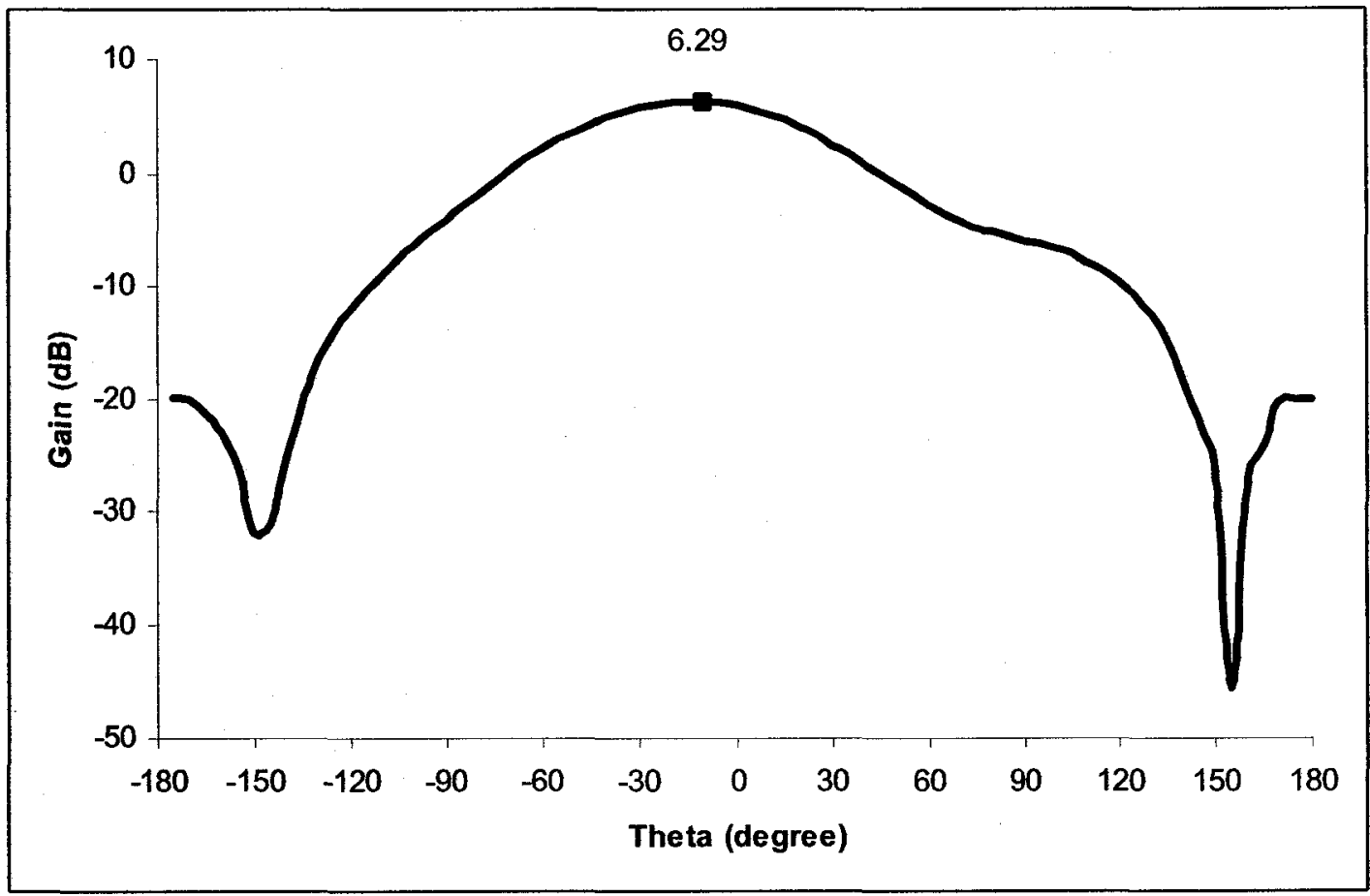

(b)

Figure 5.8: First Antenna radiation pattern for L5;

(a) $\phi=0^{\circ}$, (b) $\phi=90^{\circ}$.

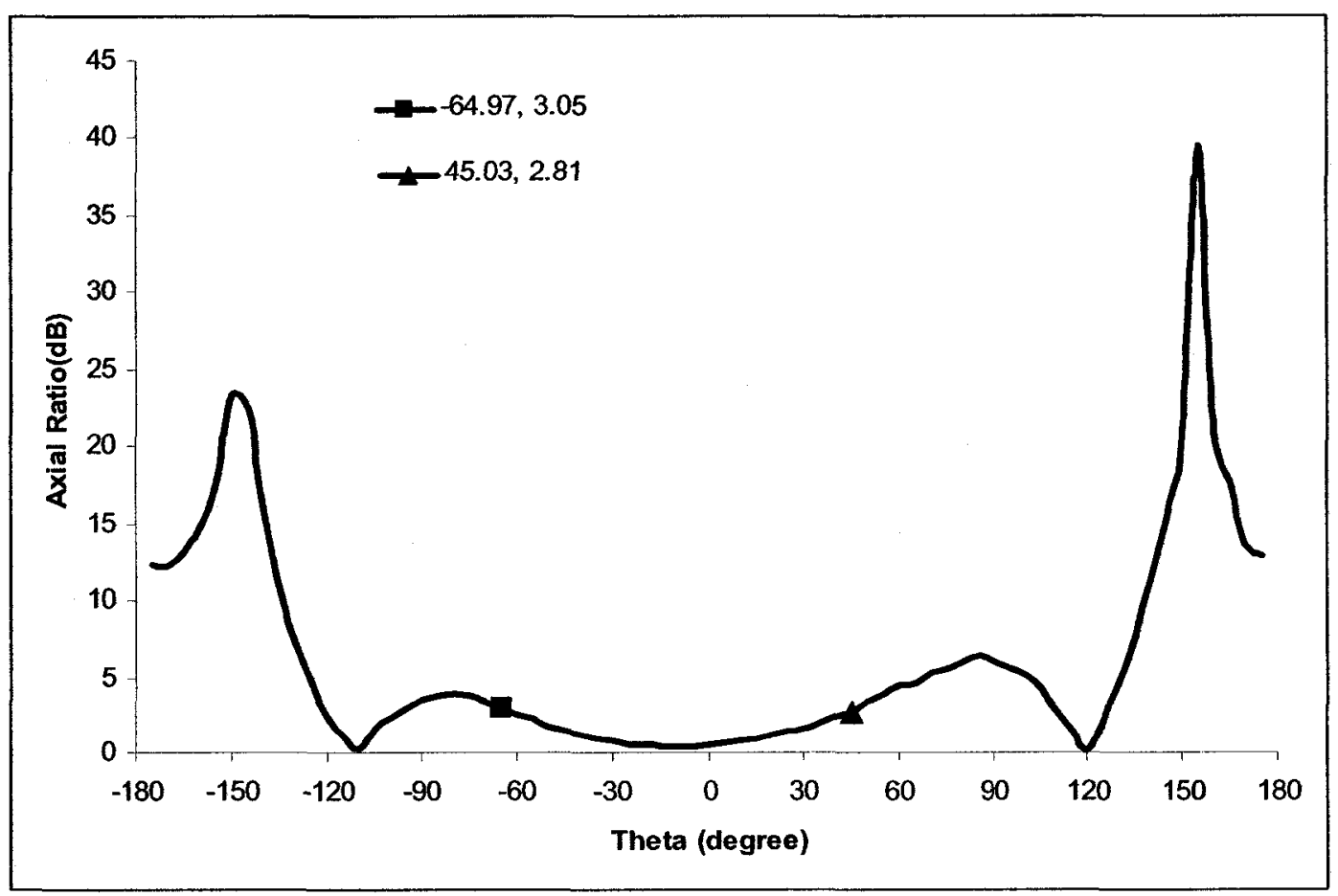

Figure 5.9: Axial ratio vs. theta in $\phi=0^{\circ}$ plane for L5. 
Table 6.1 represents a comparison of simulation and tested results. Looking at this table shows that the simulation and tested results are similar and there is no significant difference between any of the characteristics. We can also note the improvement in the axial ratio after introducing the $100 \Omega$ resistors.

\begin{tabular}{|c|c|c|c|c|c|c|c|c|c|c|}
\hline & \multicolumn{2}{|c|}{$\begin{array}{c}\text { Return Loss } \\
\text { (dB) }\end{array}$} & \multicolumn{2}{|c|}{ Gain (dB) } & \multicolumn{2}{c|}{$\begin{array}{c}\text { Axial Ratio } \\
\text { (dB) }\end{array}$} & \multicolumn{2}{c|}{$\begin{array}{c}\text { HPBW } \\
\text { (degrees) }\end{array}$} & \multicolumn{2}{c|}{$\begin{array}{c}\text { CPBW } \\
\text { (degrees) }\end{array}$} \\
\cline { 2 - 11 } & Sim & Test & Sim & Test & Sim & Test & Sim & Test & Sim & Test \\
\hline L1 & -22.8 & -16.7 & 5.33 & 5.4 & 1.5 & 1.5 & 70 & 80 & 110 & 110 \\
\hline L2 & -23.7 & -25.5 & 7.19 & 6.98 & 2.23 & 1.7 & 80 & 75 & 140 & 100 \\
\hline L5 & -18.7 & -20.5 & 6.87 & 6.07 & 2.13 & 0.9 & 90 & 90 & 150 & 110 \\
\hline
\end{tabular}

Table 6.1: Comparison of simulation and test results of first antenna 


\subsubsection{Second Antenna Test Results}

Figure 5.10 shows the tested S11 for the second designed antenna. It can be seen that this antenna is also matched at the three desired frequencies with return loss of $22.8 \mathrm{~dB}$ at $\mathrm{L} 1,-24.4 \mathrm{~dB}$ at $\mathrm{L} 2$, and $-17.79 \mathrm{~dB}$ at $\mathrm{L} 5$. Also a bandwidth of $20 \mathrm{MHz}$ for $\mathrm{L} 1$, and $160 \mathrm{MHz}$ for $\mathrm{L} 2 / \mathrm{L} 5$ is achieved.

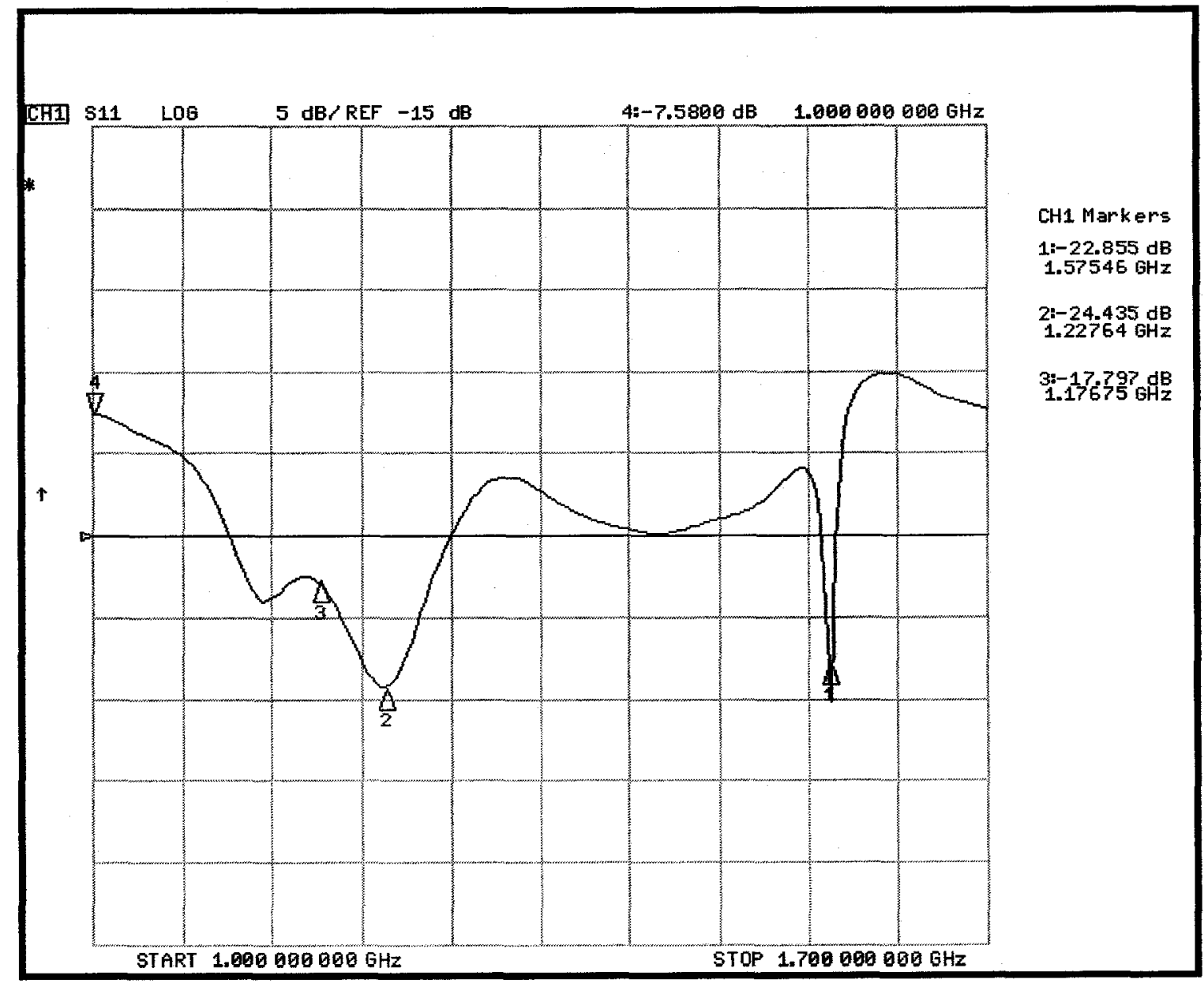

Figure 5.10: Tested return loss of the second antenna. [Agilent Network Analyzer] 
Figure 5.11 (a) and (b) show the radiation patterns of the second antenna at L1, in the $\phi=0^{\circ}$ and $\phi=90^{\circ}$ planes respectively. It can be seen that at broadside for $\phi=0^{\circ}$ a gain of $5.28 \mathrm{~dB}$ and for $\phi=90^{\circ}, 4.84 \mathrm{~dB}$ is achieved. Other characteristics of these patterns are: axial ratio of $0.9 \mathrm{~dB}$ at $\theta=0^{\circ}$, half power beamwidth (HPBW) of about $75^{\circ}$, null to null beamwidth (FNBW) of $165^{\circ}$, and a front to back ratio (FBR) of $7 \mathrm{~dB}$. Figure 5.12 shows the axial ratio vs. angle theta in $\phi=0^{\circ}$ plane. It can be seen that at $\mathrm{L} 1$, the antenna provides good CP over a beamwidth of $105^{\circ}$ (CPBW).

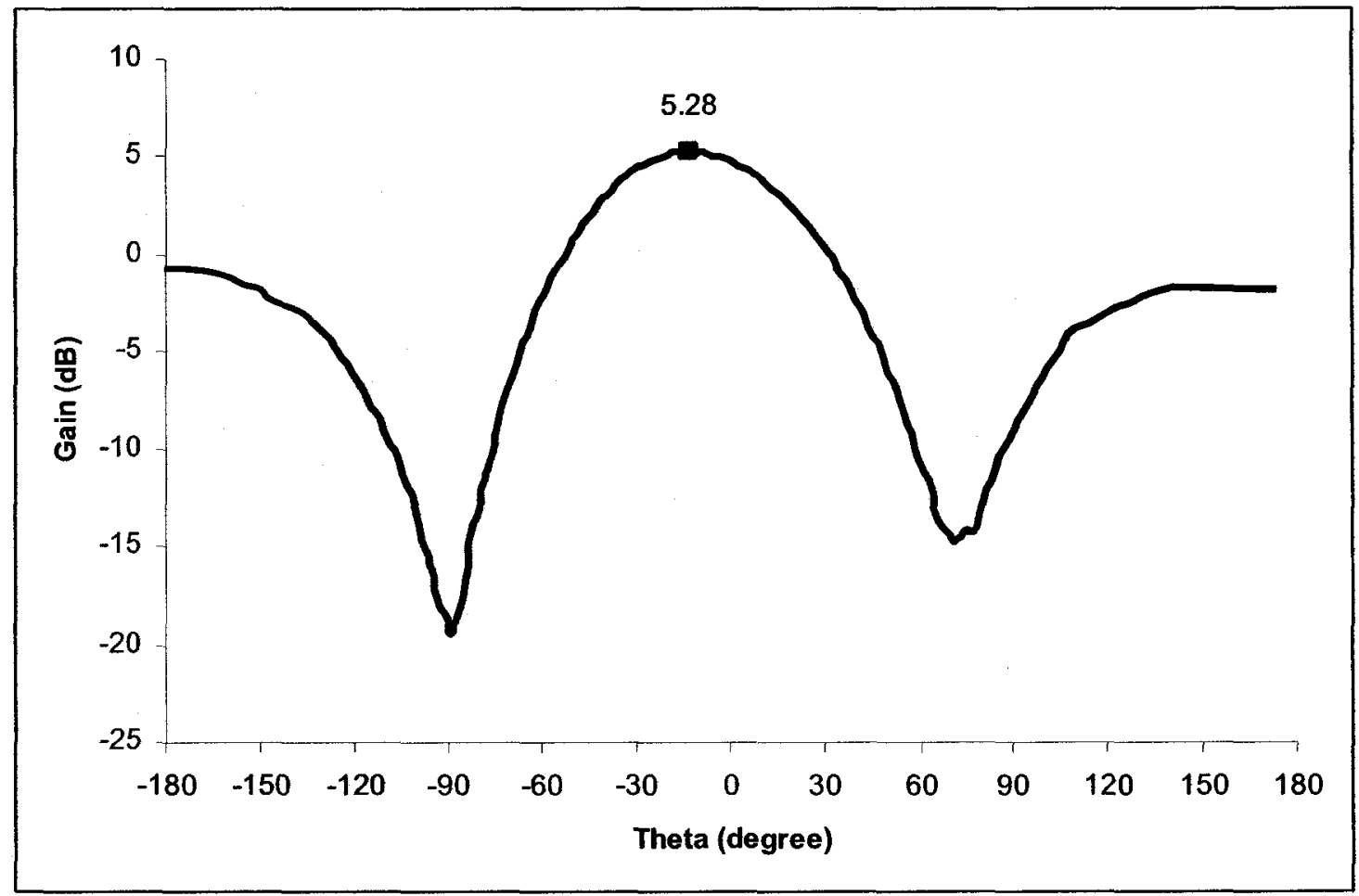

(a) 


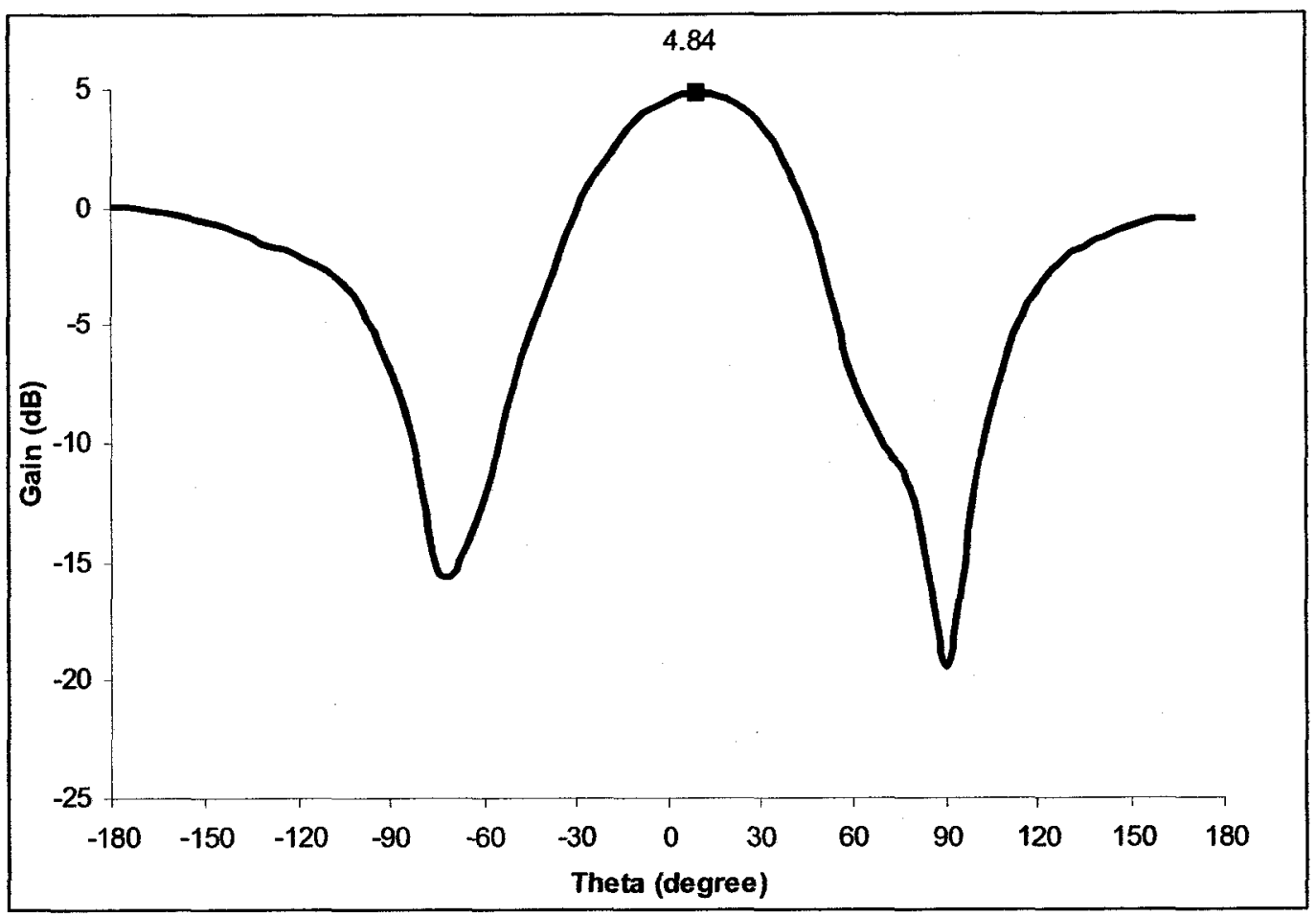

(b)

Figure 5.11: Second Antenna radiation pattern for L1;

(a) $\phi=0^{\circ}$, (b) $\phi=90^{\circ}$.

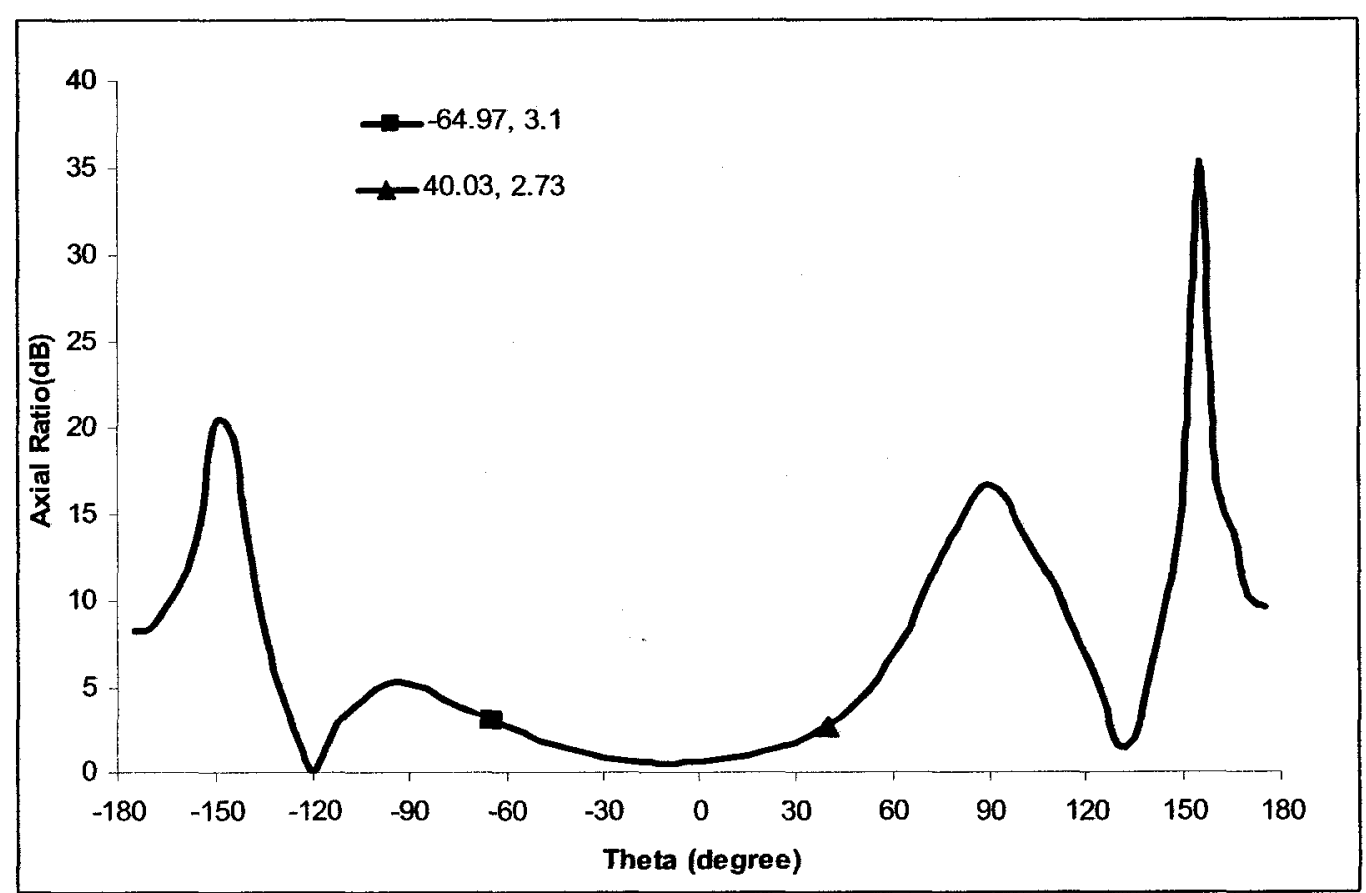

Figure 5.12: Axial ratio vs. theta in $\phi=0^{\circ}$ plane for $L 1$. 
Figure 5.13 (a) and (b) show the radiation patterns of the second antenna at L2, in the $\phi=0^{\circ}$ and $\phi=90^{\circ}$ planes respectively. It can be seen that at broadside for $\phi=0^{\circ}$ a gain of $5.6 \mathrm{~dB}$ and for $\phi=90^{\circ}, 5.22 \mathrm{~dB}$ is achieved. Other characteristics of these patterns are: axial ratio of $2.3 \mathrm{~dB}$ at $\theta=0^{\circ}$, HPBW of about $90^{\circ}, \mathrm{FNBW}$ of $210^{\circ}$, and FBR of $13 \mathrm{~dB}$. Figure 5.14 shows the axial ratio vs. angle theta in $\phi=0^{\circ}$ plane. It can be seen that at L2, the antenna provides good CP over a beamwidth of $135^{\circ}$. Also, the effect of finite size ground plane is visible in these patterns, which result in larger FNBW.

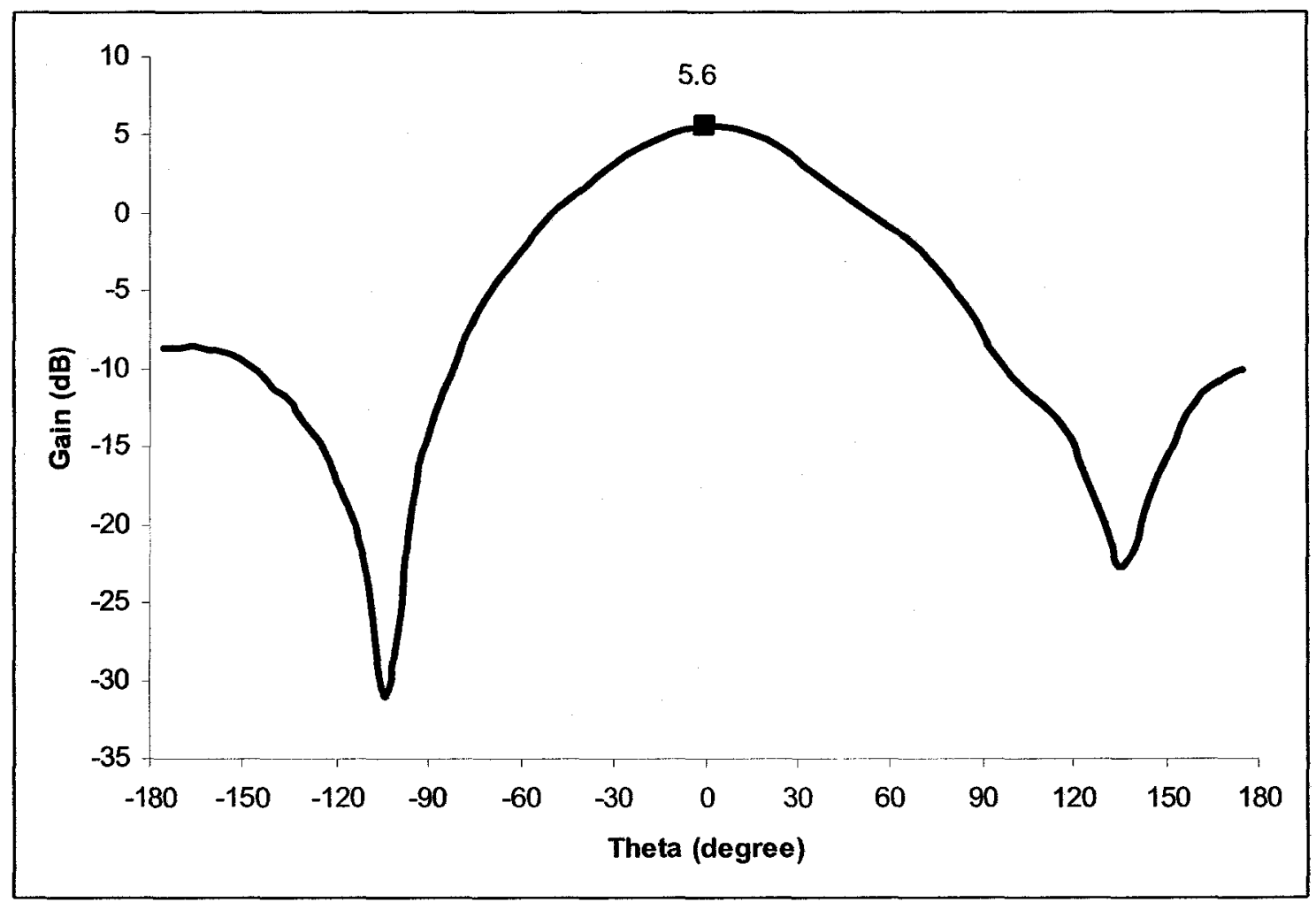

(a) 


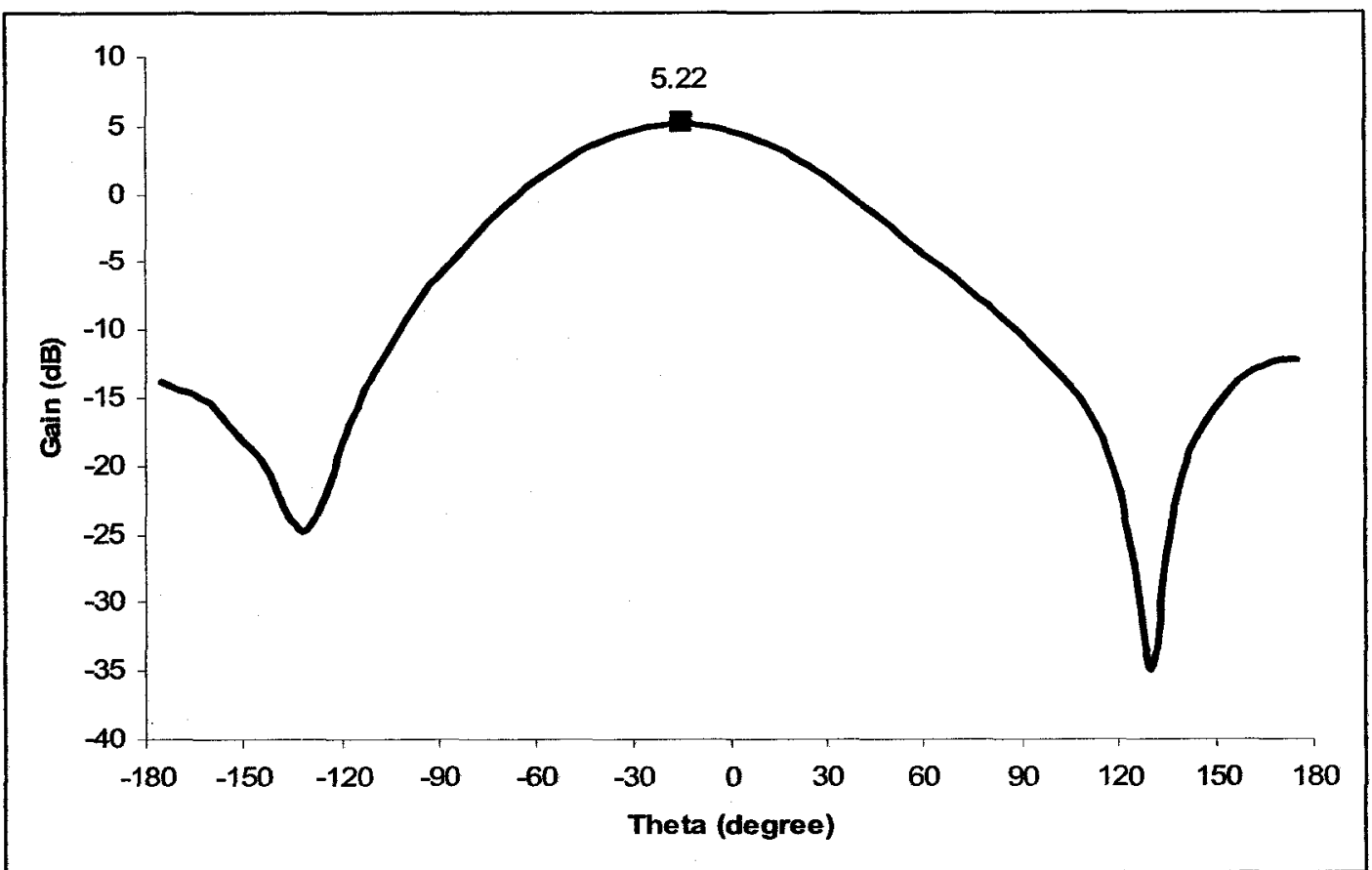

(b)

Figure 5.13: Second Antenna radiation pattern for L2;

(a) $\phi=0^{\circ}$, (b) $\phi=90^{\circ}$.

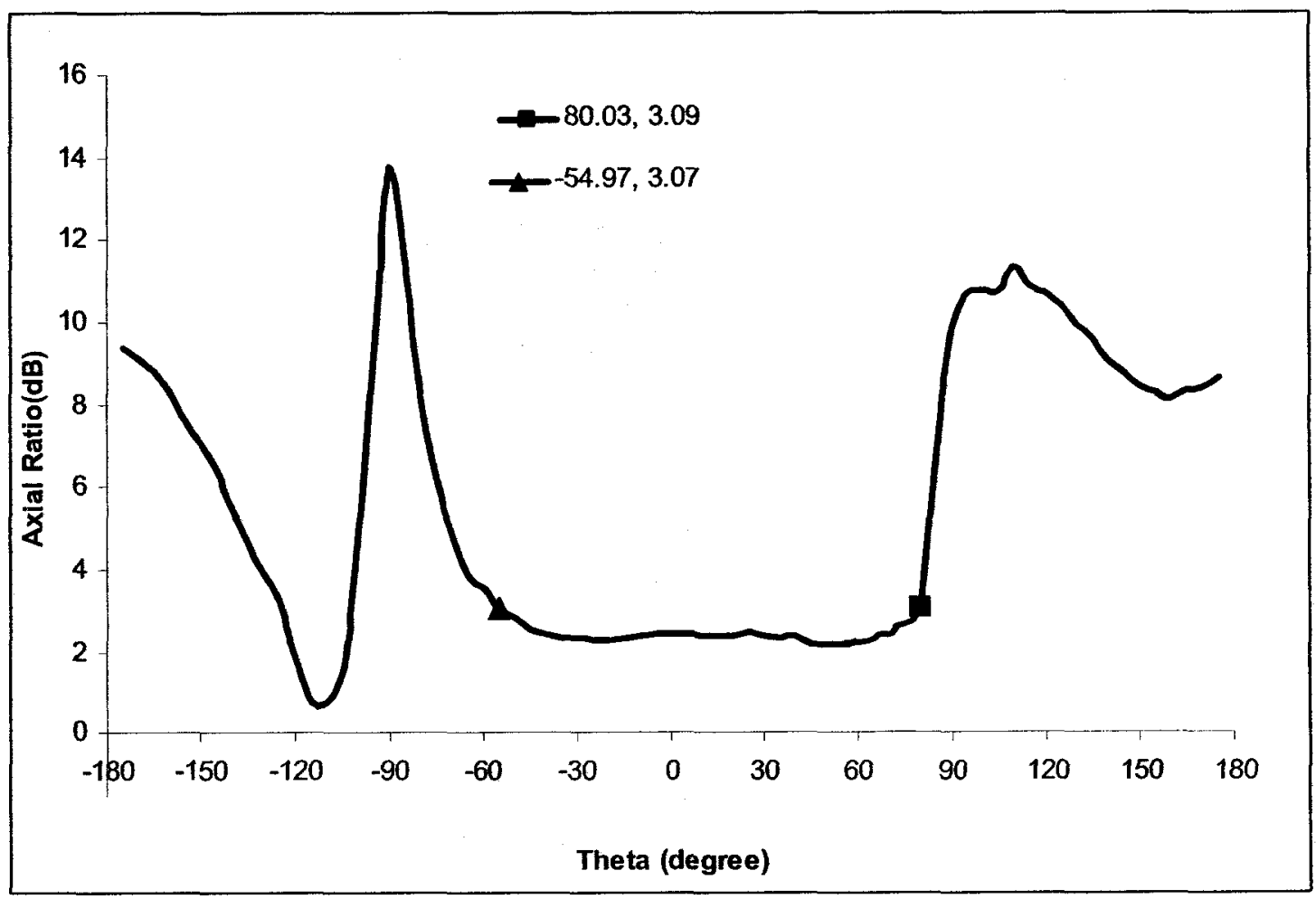

Figure 5.14: Axial ratio vs. theta in $\phi=0^{\circ}$ plane for L2. 
Figure 5.15 (a) and (b) show the radiation patterns of the second antenna at L5, in the $\phi=0^{\circ}$ and $\phi=90^{\circ}$ planes respectively. It can be seen that at broadside for $\phi=0^{\circ}$ a gain of $5.07 \mathrm{~dB}$ and for $\phi=90^{\circ}, 4.95 \mathrm{~dB}$ is achieved. Other characteristics of these patterns are: axial ratio of $0.7 \mathrm{~dB}$ at $\theta=0^{\circ}, \mathrm{HPBW}$ of about $90^{\circ}, \mathrm{FNBW}$ of $270^{\circ}$, and FBR of $15 \mathrm{~dB}$. Figure 5.16 shows the axial ratio vs. angle theta in $\phi=0^{\circ}$ plane. It can be seen that at L5, the antenna provides good CP over a beamwidth of $110^{\circ}$. Also the effect of finite size ground plane is visible in these patterns, which result in an increase in FNBW.

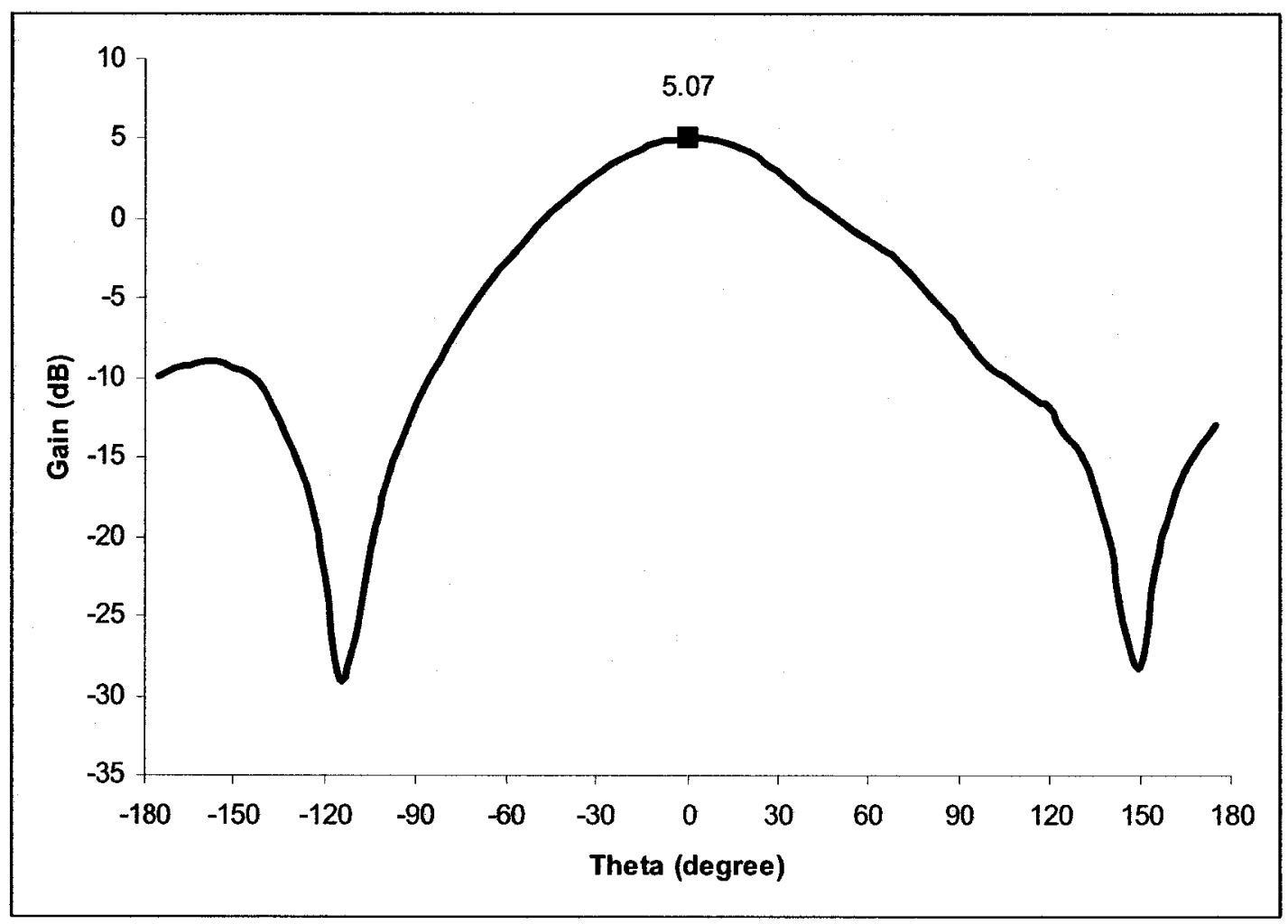

(a) 


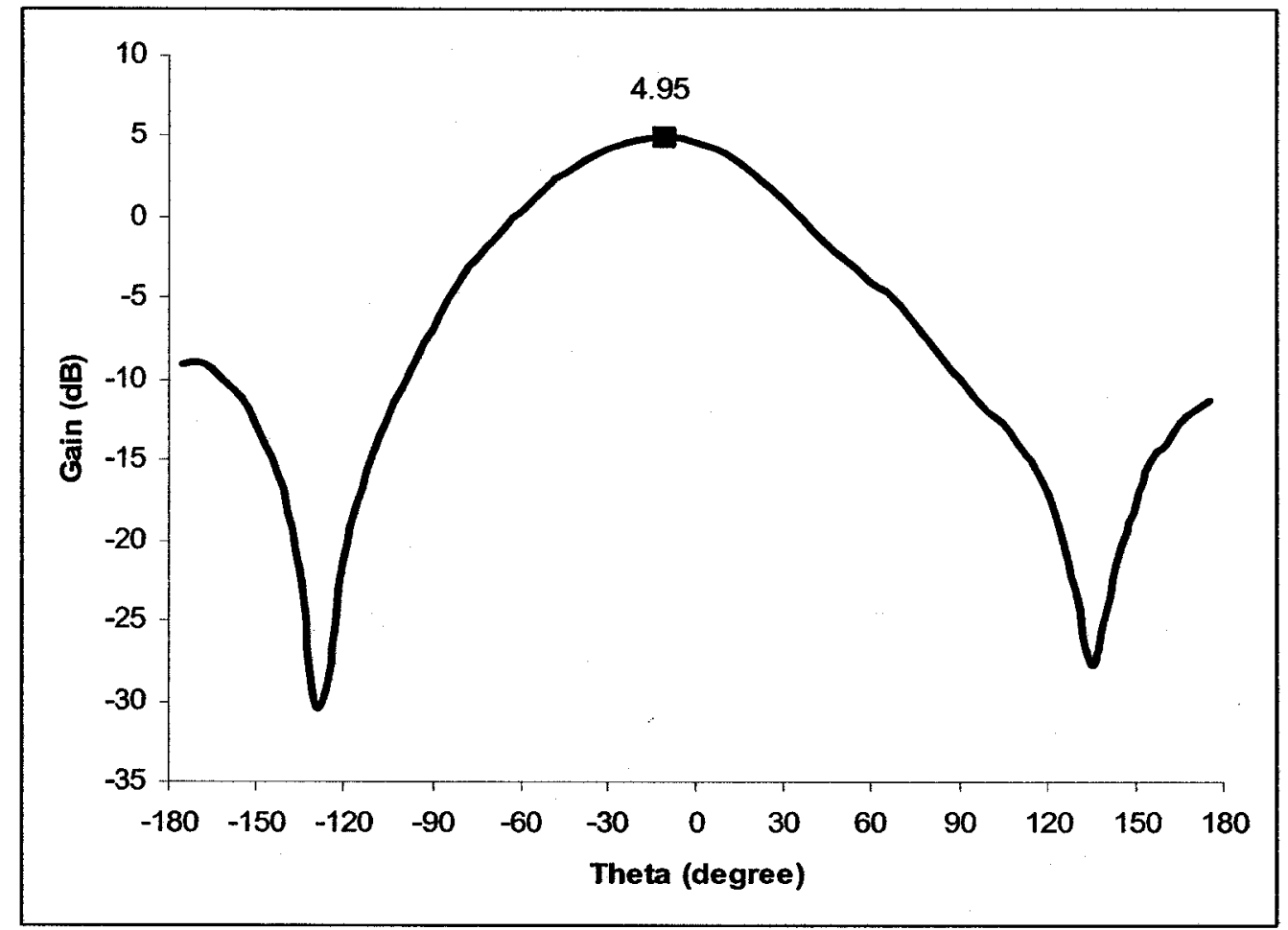

(b)

Figure 5.15: Second Antenna radiation pattern for L5;

(a) $\phi=0^{\circ}$, (b) $\phi=90^{\circ}$.

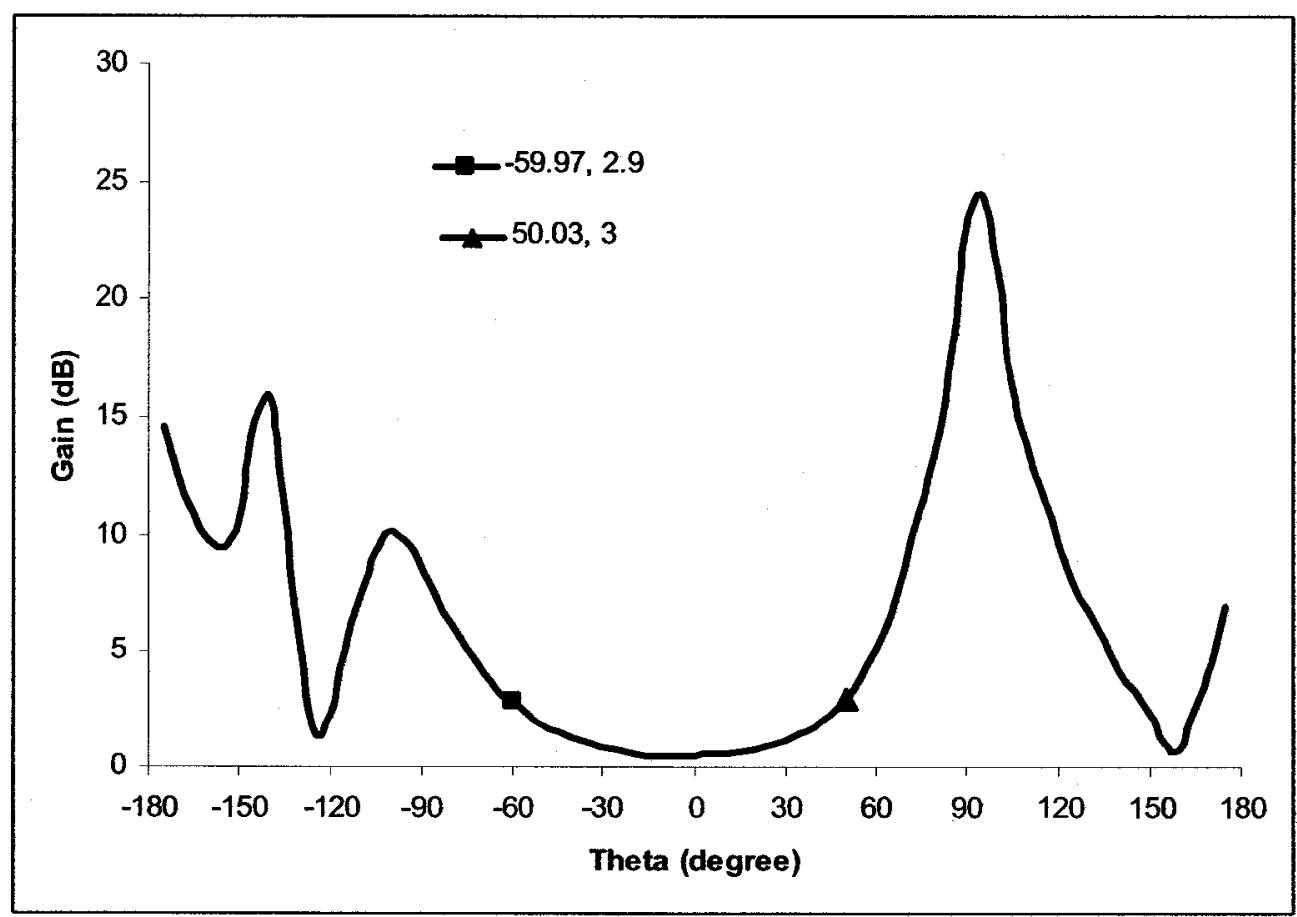

Figure 5.16: Axial ratio vs. theta in $\phi=0^{\circ}$ plane for L5. 
Table 6.2 represents a comparison of simulation and tested results. Looking at this table shows that the simulation and tested results are similar and there is no significant difference between any of the characteristics. We can also note the improvement in the axial ratio after introducing the $100 \Omega$ resistors.

\begin{tabular}{|c|c|c|c|c|c|c|c|c|c|c|}
\hline \multirow{2}{*}{ Freq } & \multicolumn{2}{|c|}{$\begin{array}{c}\text { Return } \\
\text { Loss (dB) }\end{array}$} & \multicolumn{2}{|c|}{ Gain (dB) } & \multicolumn{2}{c|}{$\begin{array}{c}\text { Axial Ratio } \\
\text { (dB) }\end{array}$} & \multicolumn{2}{c|}{$\begin{array}{c}\text { HPBW } \\
\text { (degrees) }\end{array}$} & \multicolumn{2}{c|}{$\begin{array}{c}\text { CPBW } \\
\text { (degrees) }\end{array}$} \\
\cline { 2 - 11 } & Sim & Test & Sim & Test & Sim & Test & Sim & Test & Sim & Test \\
\hline L1 & -17.7 & -22.8 & 5.33 & 5.28 & 1.6 & 0.9 & 72 & 75 & 90 & 105 \\
\hline L2 & -13.1 & -24.4 & 5.68 & 5.6 & 2.62 & 2.3 & 125 & 90 & 130 & 135 \\
\hline L5 & -13.4 & -17.8 & 5.48 & 5.07 & 2.69 & 0.5 & 115 & 90 & 140 & 110 \\
\hline
\end{tabular}

Table 6.2: Comparison of simulation and test results of second antenna 


\subsection{Conclusion:}

This chapter provided the fabrication process and the test results of the two designed antennas. The first antenna was fabricated without any flaws but the second antenna fabrication failed in the bonding stage due to the large difference in the coefficient of thermal expansion of Rogers TMM 10i and Arlon CLTE. Both antennas were tested in terms of return loss and radiation patterns and provided similar results to the simulation. The only significant difference between the tested and simulation is in FNBW, which appears more at L2 and L5. As discussed in section 3.4.6 this is due to the finite ground plane, which was not considered in simulation. Overall results of these antennas satisfy the design requirements and are capable of operating individually or in array configuration for the purpose of beam/null steering. 


\section{Chapter 6}

\section{$-$ \\ Conclusions And Future Work}

\subsection{Summary}

This thesis has investigated the technique of stacking microstrip antennas to achieve dual frequency operation for GPS frequencies L1 and L2/L5. The patches were fed by a parallel coplanar microstrip feedline, which provides accurate amplitude and phase for circular polarization. Energy is electromagnetically coupled through an aperture in the ground plane. This configuration consists of three resonators, two patches and the aperture. There is a mutual coupling between each resonator and by adjusting the resonator size, substrate thickness and permittivity the mutual coupling can be adjusted. The coupling between the two patches is dependant to the relative size of the patches and also whether the top patch is smaller or larger than the bottom patch. The relative patch sizes must be adjusted for proper impedance match. Since the lower frequency band L2/L5 requires larger bandwidth than the L1 band, the top patch was chosen to be larger. The design of this antenna was a tedious process and required numerous empirical tunings due to the number of interacting parameters. 
Two similar antenna structures were designed using different substrate material. The first antenna was designed so that the fabrication and material cost is minimal. A second design was carried out to reduce the size of the first antenna. This reduction in size required different substrate materials with higher permittivity. The second antenna is $30 \%$ smaller compared to the first antenna. Both antennas were fabricated and tested and satisfy the design requirements.

\subsection{Future Work}

The designed aperture-coupled stacked patch configuration suffers from high back lobe radiation in L1 frequency band. Although operation is satisfactory for both antennas in this regard, further effort could be directed to improve this unwanted radiation. One way to overcome the back lobe radiation is by shielding the back of the antenna by another virtual ground plane. Also, the aperture length could be designed so that its resonant frequency is further apart from $\mathrm{L} 1$ band. Both of these methods require a complete revision in all design parameters and may degrade other characteristics of the antenna.

The designed antennas can be used in a linear or planar array configuration for the purpose of beam/null steering and could serve as an antenna anti-jamming device. Several attempts to simulate a 2 by 2 array failed due to lack of sufficient computer memory. Also the fabrication of the second antenna was unsuccessful for array formation. A second attempt for fabrication could be done without using bonding sheets. 


\section{REFERENCES}

[1] R. Garg, P. Bhartia, I. Bahl, A. Ittipiboon, "Microstrip Antenna Design Handbook", Artech House, MA, 2001.

[2] K.L. Wong, "Compact and Broadband Microstrip Antennas", John Wiley \& Sons, NY, 2002.

[3] J.F. Zurcher, F.E. Gardiol, "Broadband Patch Antennas”, Artech House, MA, 1995.

[4] E. D. Kaplan, "Understanding GPS: Principals and Applications", Artech House, MA, 1996.

[5] D.M. Pozar, S.M. Duffy, "A Dual-Band Circularly Polarized Aperture-Coupled Stacked Microstrip Antenna", IEEE Trans. Antenna and Propagation, Vol. 45, No. 11, November 1997.

[6] D.M. Pozar, S.D. Targonski, "Design of a Wide-Band Circularly Polarized ApertureCoupled Microstrip Antennas", IEEE Trans. Antenna and Propagation, Vol. 41, No. 2, February 1993.

[7] D.M. Pozar, S.D. Targonski, R.B. Waterhouse, "Design of a Wide-Band ApertureStacked Patch Microstrip Antennas", IEEE Trans. Antenna and Propagation, Vol. 46, No. 9, September 1998.

[8] D. Gray, M. Trinkle, "Overview of GPS Receiver Anti-Jam Technologies", Cooperative Research Centre for Sensor Signal and Information Processing, Adelaide University, Australia, April 2001. 
[9] M. Trinkle, "The Comparative Analysis of GPS Receiver Anti-Jam Technologies", Cooperative Research Centre for Sensor Signal and Information Processing, Australia, September 2001.

[10] J. F. Raquet, "Short Course: GPS 101", Joint Navigational Conference, Las Vegas, NV, April 2003

[11] C.A. Balanis, “Antenna Theory: Analysis and Design", John Wiley \& Sons, NY, 1997.

[12] A. Petosa, “Antenna and Arrays: ELEC5607", Carleton University, ON, 2004

[13] Y.B. Gan, C.P. Chua, L.W. Li, "An Enhanced Cavity Model for Microstrip Antennas", Microwave and Optical Technology Letters, Vol. 40, No. 6, March 2004.

[14] K.R. Carver, J.W. Mink, "Microstrip Antenna Technology", IEEE Trans. Antenna and Propagation, Vol. AP-29, January 1981.

[15] J. James, P.S. Hall, "Handbook of Microstrip Antennas", Peter Peregrinus, London, UK, 1989.

[16] D.M. Pozar, "Rigorous Closed-Form Expressions for the Surface Wave Loss in Printed Antennas", Electron. Lett., Vol. 26, 1990

[17] D.R. Jackson, N.G Alexopoulos, "Simple Approximate Formulas for Input Resistant, Bandwidth and Efficiency of a Resonant Rectangular Patch", IEEE Trans. Antenna and Propagation, Vol. AP-39, 1991.

[18] J.R. James, A. Henderson, "High Frequency Behavior of Microstrip Open Circuit Terminations", IEE J., Microwaves, Optics and Acoustics, Vol. 3, 1979. 
[19] E. Lier, K.R. Jacobson, "Rectangular Microstrip Antennas with Infinite and Finite Ground Plane Dimensions", IEEE Trans. Antenna and Propagation, Vol. AP-31, 1983.

[20] J. Huang, "The Finite Ground Plane Effect on rectangular Patch Radiation Pattern", IEEE Trans. Antenna and Propagation, Vol. AP-31, 1983.

[21] D.M. Pozar, “Microstrip Antennas”, Proc. IEEE, Vol. 80, 1992.

[22] G.A Kyriacou, A.A. Mavrides, O. Breinbjerg, J.N. Sahalos, “A Design Procedure for Aperture Coupeled Microstrip Antennas Based on Approximate Equivalent Networks", work in collaboration Demokritos Univ. Denmark

[23] M. Himidi, J.P. Daniel, C.Terret, “Transmission Line Analysis of Aperture- Coupled Microstrip Antenna", Electron. Lett., Vol. 25, 1989.

[24] B.N. Das, K.K. Joshi, "Impedance of a Radiating Slot in the Ground Plane of a Microstripline", IEEE Trans. Antenna and Propagation, Vol. AP-30, 1982.

[25] A. Adrian, D.H. Schaubert, "Dual Aperture-coupled Microstrip Antennas for Dual or Circular polarization”, Electron. Lett., Vol. 23, 1987.

[26] D.M. Pozar, “Microwave Engineering”, John Wiley \& Sons, NY, 1998.

[27] K.R. McKay, “A Stacked Microstrip Antenna for Mobile Satellite Communication”, Carleton University, Ottawa, Ontario, 1994. 


\section{Appendix A}

Agilent $8720 \mathrm{ES}$ network analyzer is used in this experiment to make the required S-parameter measurements. Prior to making measurements, the network analyzer must be calibrated using proper open, short, and a $50 \Omega$ impedance standards. Once the calibration is verified, the Antenna under test (AUT) is connected to port 1 of the network analyzer. Using the menus on the analyzer, the frequency range for the test can be selected and various options are available for viewing the results.

For the radiation pattern measurement, a computer is connected to both the Agilent Network Analyzer and the Orbit AL-4806 Positioner. The FR959 software package controls both pieces of equipment. The operation of the system is as follows (ELEC4503 lab manual):

1. The network analyzer sends an RF signal to the transmitting antenna at a specified frequency or list of frequencies via port 1.

2. The signal is then radiated to the antenna under test (AUT).

3. The AUT receives the signal and sends it to port 2 of the network analyzer after passing through an HP 87405A preamplifier.

4. The network analyzer measures the magnitude of the transmission coefficient (S21) as a function of the AUT's angular position. The role of the Orbit AL-4806 is to control the turntable, which rotates the AUT during the measurement. The rotation is user determined to go from a certain angle to another (i.e. -180 degrees to +180 degrees). 
Depending on how the antenna is oriented this could mean a rotation in the antenna's azimuth or elevation planes. Before getting any measurements done the anechoic chamber is calibrated using a horn antenna with known gain in different frequencies. 


\section{Appendix B}
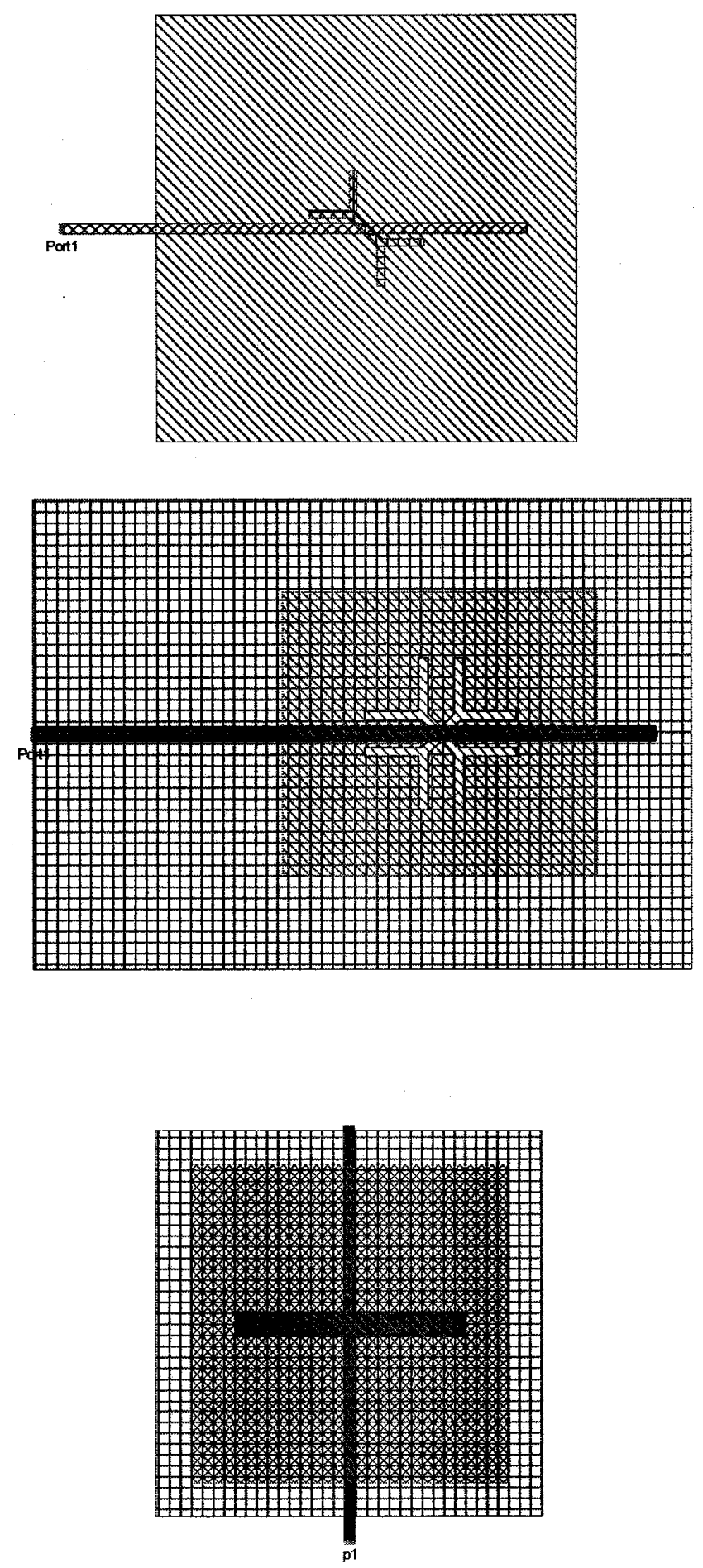